Florida International University FIU Digital Commons

\title{
Enhancing Algal Biomass and Lipid Production through Bacterial and Fungal Co-Culture
}

Erwin David Berthold

Florida International University, deberthold@gmail.com

DOI: $10.25148 /$ etd.FIDC000792

Follow this and additional works at: https:// digitalcommons.fiu.edu/etd

Part of the Oil, Gas, and Energy Commons, Other Environmental Sciences Commons, and the Sustainability Commons

\section{Recommended Citation}

Berthold, Erwin David, "Enhancing Algal Biomass and Lipid Production through Bacterial and Fungal Co-Culture" (2016). FIU Electronic Theses and Dissertations. 2563.

https://digitalcommons.fiu.edu/etd/2563 


\title{
FLORIDA INTERNATIONAL UNIVERSITY
}

\author{
Miami, Florida
}

\section{ENHANCING ALGAL BIOMASS AND LIPID PRODUCTION THROUGH BACTERIAL AND FUNGAL CO-CULTURE}

\author{
A thesis submitted in partial fulfillment of \\ the requirements for the degree of \\ MASTER OF SCIENCE \\ in \\ ENVIRONMENTAL STUDIES \\ by \\ Erwin David Berthold
}


To: Dean Michael R. Heithaus

College of Arts, Sciences and Education

This thesis, written by Erwin David Berthold and entitled Enhancing Algal Biomass and Lipid Production through Bacterial and Fungal Co-Culture, having been approved in respect to style and intellectual content, is referred to you for judgment.

We have read this thesis and recommend that it be approved.

Krishnaswamy Jayachandran

Miroslav Gantar, Co-Major Professor

Kateel G. Shetty, Co-Major Professor

Date of Defense: July 6, 2016

The thesis of Erwin David Berthold is approved.

Dean Michael R. Heithaus
College of Arts, Sciences and Education

College of Arts, Sciences and Education

Andrés G. Gil

Vice President for Research and Economic Development and Dean of the University Graduate School 


\section{ACKNOWLEDGMENTS}

I would like to acknowledge partial funding by USDA-NIFA-National Needs Fellows-2013-38420-30804. 


\title{
ABSTRACT OF THE THESIS \\ ENHANCING ALGAL BIOMASS AND LIPID PRODUCTION THROUGH \\ BACTERIAL AND FUNGAL CO-CULTURE
}

\author{
by
}

\author{
Erwin David Berthold
}

Florida International University, 2016

Miami, Florida

\section{Professor Kateel G. Shetty, Co-Major Professor \\ Professor Miroslav Gantar, Co-Major Professor}

This thesis investigates the effects of co-culturing microorganisms including 37 yeast, 38 bacteria, nine diazotrophic cyanobacteria, and three fungi on biomass and lipid production in fresh- and saltwater algae. Algal lipid content was measured using Nile Red method and gravimetric techniques. Among the algal strains tested, freshwater Coelastrum sp. 46-4, and saltwater Cricosphaera sp. 146-2-9, showed enhanced biomass yield and lipid content in response to co-culture with bacteria, cyanobacteria, and fungi. While co-culture with yeast caused inhibition of algal productivity, no difference in algal productivity was observed between nitrogen-free diazotrophic cyanobacterial co-culture and nitrogen-replete monoalgal culture. Results indicated that extracellular compounds from the freshwater bacteria Pseudomonas stutzeri and marine fungus Fusarium sp. significantly account for stimulation of lipid accumulation within algal cells, while co-cultivation with live microorganism cells stimulated biomass production in algae. 


\section{TABLE OF CONTENTS}

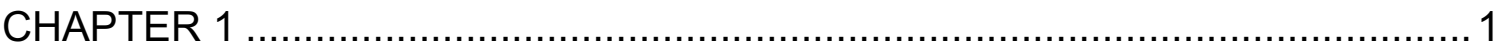

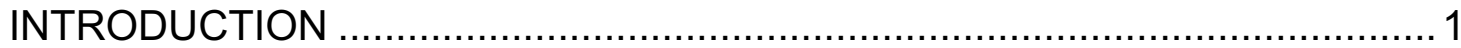

1.1 Rationale for improving algae biofuel outputs ................................... 1

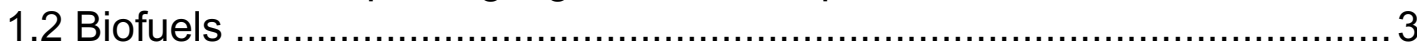

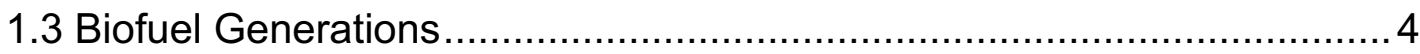

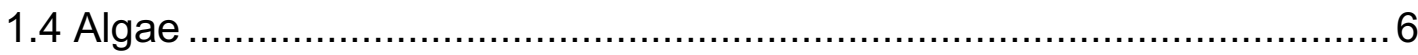

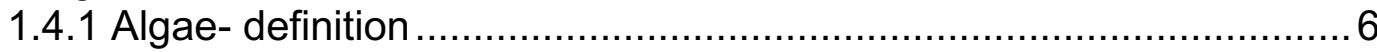

1.4.2 Algae biofuel process .........................................................

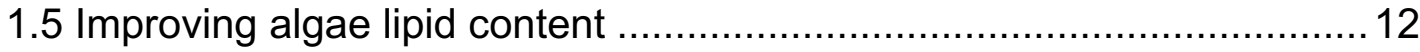

1.5.1 Cultivation stress and genetic manipulations ............................. 13

1.5.2 Co-Culturing of algae with microorganisms ................................. 15

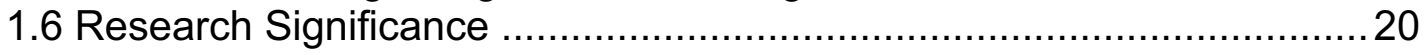

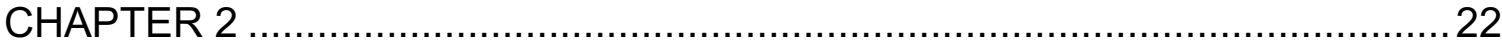

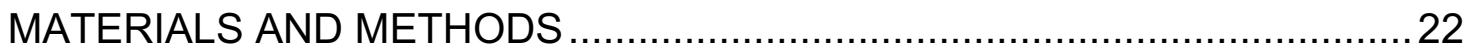

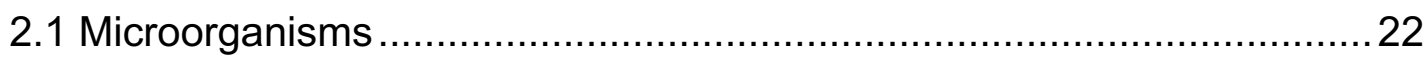

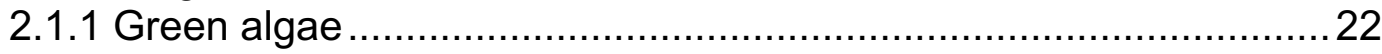

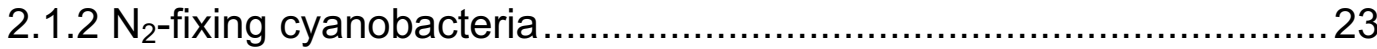

2.1.3 Isolation and purification of algae .............................................2 24

2.1.4 Isolation of yeast, fungi, and bacteria ..........................................25

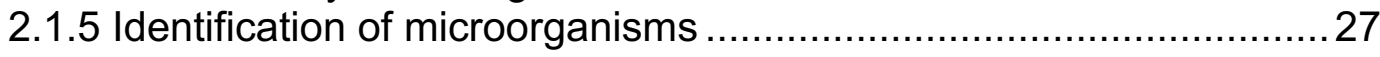

2.1.5.1 Identification of algae Isolates ........................................... 27

2.1.5.2 Identification of bacterial and fungal Isolates .........................2 27

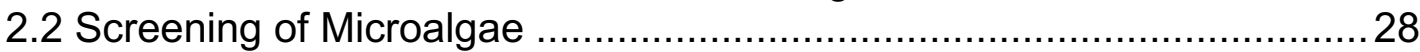

2.2.1 Growth and Biomass Productivity of Freshwater Microalgae ..........28

2.2.2 Lipid productivity of Freshwater Microalgae ................................28

2.2.3 Biomass and Lipid Productivity of Marine Microalgae ......................30

2.3 Screening for high biomass and lipid production in co-cultures ..............31

2.3.1 Growth and lipid content of freshwater microalgae and yeast.........31

2.3.2 Growth and lipid content of freshwater microalgae with bacteria ....32

2.3.3 Growth and lipid content of freshwater microalgae and $\mathrm{N}_{2}$-fixing

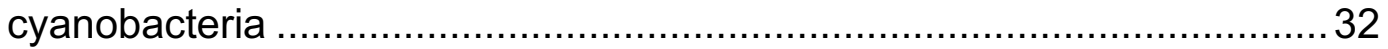

2.3.4 Growth and lipid content of marine microalgae and fungi ................33

2.4 Biomass and lipid productivity of co-cultures ......................................... 33

2.4.1 Co-cultivation vs. bacteria-cell free filtrate ..................................... 34

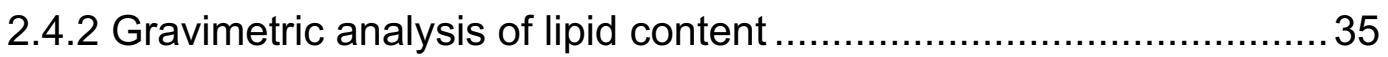

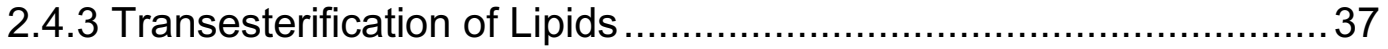

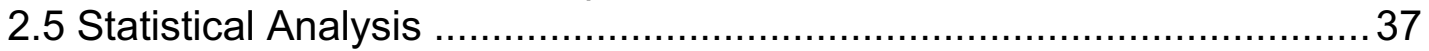

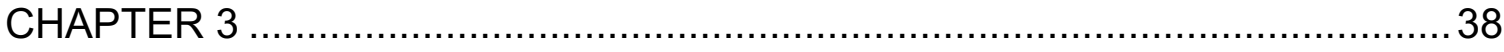

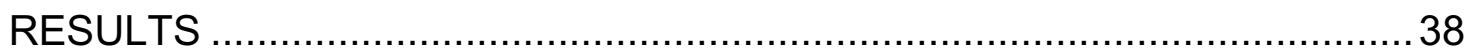

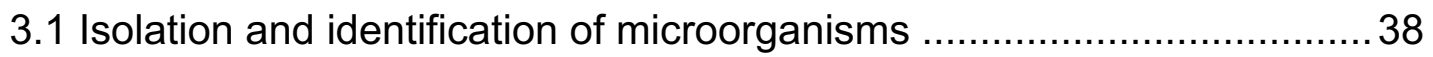




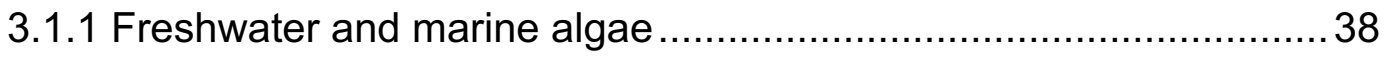

3.1.2 Bacteria, Yeast, and Fungi .................................................. 41

3.2 Freshwater algae biomass and lipid productivity ............................... 42

3.3 Marine algae biomass and lipid productivity ...................................... 44

3.3 Screening co-culture experiments................................................. 47

3.3.1 Coelastrum sp. 46-4 and yeast, bacteria, and $\mathrm{N}_{2}$-fixing

Cyanobacteria co-cultures ..................................................4 4

3.3.2 Co-culture of Cricosphaera sp. 146-2-9 with fungi ..........................54

3.4 Co-culture of Coelastrum sp. 46-4 vs. bacterial-cell free filtrate .............56

3.5 Co-culture of Coelastrum sp. 46-4 and Nostoc sp. 47-2-1 ...................61

3.6 Co-culture of Cricosphaera sp. 146-2-9 vs. fungal cell-free filtrate .........64

3.7 Gravimetric lipid analysis of Coelastrum sp. 46-4 and Cricosphaera

sp. $\quad 146-2-9$ in mono- and co-cultures and microorganism-cell free

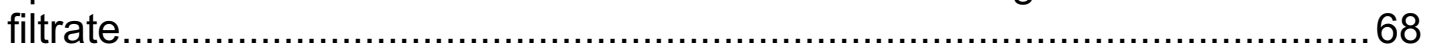

3.7.1 Co-cultures of Coelastrum sp. 46-4 with Pseudomonas stutzeri.....68

3.7.2 Co-cultures of Coelastrum with Nostoc sp. 47-2-1 .........................69

3.7.3 Co-culture of Cricosphaera sp. 146-2-9 with Fusarium sp.

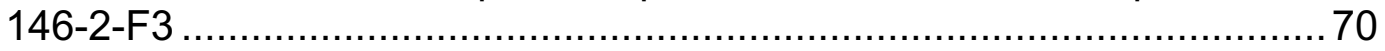

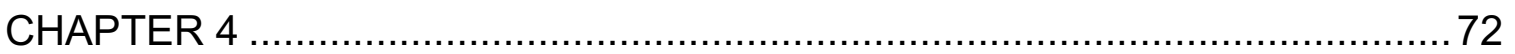

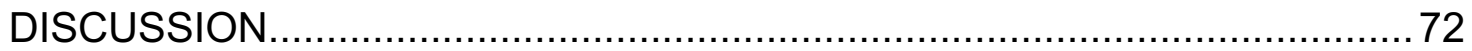

4.1 Using South Florida algae for biofuels .......................................... 72

4.2 Screening of co-cultures of algae and microorganisms ....................... 74

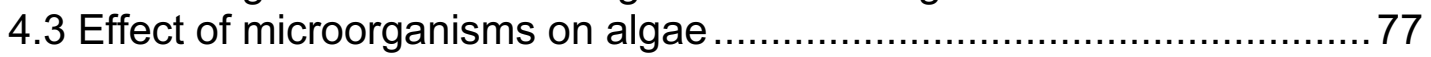

4.4 Gravimetric analysis of biomass and lipid content .............................. 79

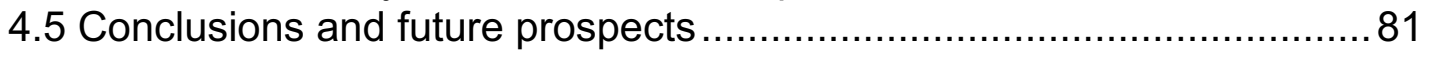

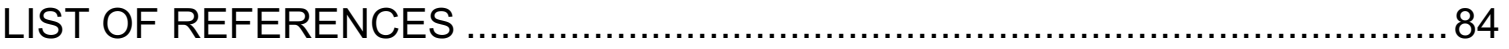




\section{LIST OF TABLES}

TABLE

PAGE

Table 1 Chlorophyceae algae used in this research........................................38

Table 2 Isolated marine algae (BG11 marine) ….......................................... 40

Table 3 Biomass and lipid percentages of 22 Chlorophyceae green algae.........43

Table 4 Percent difference over monocultures in the biomass and lipid content of Coelastrum sp. $46-4$ with 37 yeast strains over two weeks ....... 49

Table 5 Percent difference over monocultures in the biomass content of Coelastrum sp. 46-4 with 38 bacterial strains over two weeks 


\section{LIST OF FIGURES}

FIGURE

PAGE

Figure 1 The process of transesterification in manufacturing biodiesel ................. 3

Figure 2 Schematic overview of the algae-based biofuel process ....................... 8

Figure 3 Map of the South Florida region indicating sampling sites ..................23

Figure 4 Diazotrophic cyanobacteria used in this research: a) b) Tolypothrix sp. 30-1-4, 33-4, c) Nostoc sp. 47-2-1, d) e) Calothrix sp. 69-4, 113-9, f) Nostoc sp. 113-10. 1000X magnification; scale bar indicates $10 \mu \mathrm{m}$

Figure 5 Triolein lipid standard curve used to quantify lipid concentration within algal cell culture

Figure 6 Freshwater algae used in this research; a) b) Coelastrum sp. 5-1, 46-4, c) Stigeoclonium sp. 64-8, d) Selenastrum sp. 64-10, e) Dictyococcus sp. 64-12, f) Chlorococcum sp. 103-6, g) Oocystis sp. 108-5, h) i) Chlorococcum sp. 142-5-2, 143-1, j) Coelastrum sp. 143-5, k) Scenedesmus sp. 143-4, I) Chlorococcum sp. 143-6, m) Coelastrum sp. 108-4, n) o) Scenedesmus sp. 145-2, 145-3, p) Dictyochloropsis sp. 145-7, q) Oocystis sp. 148-1-1, r) Scenedesmus sp. 148-5-2, s) Chlorella sp. 150-1-1, t) Characium sp. 152-3, u) Chlorella sp. 155-1. 1000X magnification; Scale bar indicates $10 \mu \mathrm{m}$ 40

Figure 7 Marine algae isolated for this research; a) Tetraselmis sp. 146-2-1, b) Chlorococcum sp. 146-2-6, c) Vischeria sp. 146-2-8, d) Cricosphaera sp. 146-2-9, e) Chlamidomonas sp. 146-2-10, f) Chrysotila sp. 146-2-11, g) Eustigmatos sp. 146-2-14, h) Chlorella sp. 146-2-15, i) Vischeria sp. 146-2-16. 1000X magnification; Scale bar indicates 10 $\mu \mathrm{m}$

Figure 8 The biomass content $\left(\mathrm{g} \mathrm{L}^{-1}\right)$ of nine marine algae over twenty days. Data represents the mean of three replicates.

Figure 9 Average biomass ( $\mathrm{g} \mathrm{L}^{-1}$ dry weight) and lipid content (\% of dry biomass) of nine marine algae over 20 days of cultivation. Data represents the mean of three replicates over days $5,10,15$, and 20 ....

Figure 10 Lipid productivities ( $\mathrm{mg} \mathrm{L}^{-1} \mathrm{day}^{-1}$ ) of nine marine algae strains over twenty days. Data represents the mean of three replicates

Figure 11 Optical density $(\mathrm{a} ; 600 \mathrm{~nm})$ and lipid concentration $\left(\mathrm{b} ; \mu \mathrm{g} \mathrm{ml}^{-1}\right)$ of the control Coelastrum sp. 46-4 grown in nitrogen-supplemented $\mathrm{N}+$ medium and co-cultured with nine diazotrophic cyanobacteria in nitrogen deficient medium ( $\mathrm{N}-$ ) over 5,10 , and 15 days. Data represents the mean of three replicates 
Figure 12 Biomass ( $\mathrm{g} \mathrm{L}^{-1}$ dry weight) and lipid concentration ( $\mathrm{mg} \mathrm{g}^{-1}$ dry biomass) of Cricosphaera sp. 146-2-9 (control- C) co-cultured with three fungal species $(F 1,3,15)$ on week 2 . Data represents the mean of four replicates

Figure 13 Bright field view (left) and Nile red fluorescence (right) of Cricosphaera sp. 146-2-9 alone in medium (a) and co-cultured with three fungal species 146-2- F1, F3, F15 (b, c, d; respectively). 1000X magnification; scale bars indicate $10 \mu \mathrm{m}$

Figure 14 Biomass ( $\mathrm{g} \mathrm{L}^{-1}$ dry weight) and lipid concentration ( $\mathrm{g} \mathrm{g}^{-1}$ dry biomass) during the first (a) and second week (b) of cultivation of Coelastrum sp. 46-4 monoculture (-C) and co-culture with Pseudomonas stuzeri (- M), inoculated in bacterial cell-free filtrate (BS) and in nutrient broth (-NB). Data represents the mean of four replicates.

$\left({ }^{*}\right)$ Indicates significant differences from the control $(\alpha=0.05)$ 58

Figure 15 Bright field view (left) and Nile red fluorescence (right) of Coelastrum sp. 46-4 alone in medium (a), co-cultured with Pseudomonas stutzeri (b), in nutrient broth (c), and inoculated in bacteria cell-free filtrate (d). 1000X magnification; scale bars indicate $10 \mu \mathrm{m}$ 60

Figure 16 Biomass ( $\mathrm{g} \mathrm{L}^{-1}$ dry weight) and lipid concentration (mg g ${ }^{-1}$ dry biomass) during the first (a) and second week (b) of cultivation of Coelastrum sp. 46-4 in monocultures (-C) and co-cultured with Nostoc sp. 47-2-1 (-M). Data represents the mean of four replicates. $\left(^{*}\right)$ Indicates significant differences $(\alpha=0.05)$

Figure 17 Bright field view (left) and Nile red fluorescence (right) of Coelastrum sp. 46-4 alone in medium (a), co-cultured with Nostoc sp. 47-2-1 (b). 1000X magnification; scale bars indicate 10 $\mu \mathrm{m}$

Figure 18 Biomass ( $\mathrm{g} \mathrm{L}^{-1}$ dry weight) and lipid concentration ( $\mathrm{g} \mathrm{g}^{-1}$ dry biomass) during the first (a) and second week (b) of cultivation of Cricosphaera sp. 146-2-9 monoculture (-C) and co-culture with Fusarium sp.146-2-F3 (- M), inoculated in fungal cell-free filtrate (FS) and in marine GPY (-GPY). Data represents the mean of four replicates. $\left({ }^{*}\right)$ Indicates significant differences from the control $(\alpha=0.05)$

Figure 19 Bright field view (left) and Nile red fluorescence (right) of Cricosphaera sp. 146-2-9 alone in medium (a), co-cultured with Fusarium sp. 146-2-F3 (b), in GPY (c), and inoculated in fungal cell-free filtrate (d). 1000X magnification; scale bars indicate $10 \mu \mathrm{m}$

Figure 20 The biomass ( $\mathrm{g} \mathrm{L}^{-1}$ dry weight), total lipid and FAME content (\% yield of dry biomass) of Coelastrum sp. 46-4 in monoculture (-C), 
co-cultured with Pseudomonas stutzeri (-M), and inoculated in bacterial cell-free filtrate (-BS) and nutrient broth (-NB)

Figure 21 The biomass ( $\mathrm{g} \mathrm{L}^{-1}$ dry weight), total lipid and FAME content (\% yield of dry biomass) of Coelastrum sp. 46-4 in monoculture (-C) and co-cultured with Nostoc sp. 47-2-1 (-M)

Figure 22 The biomass ( $\mathrm{g} \mathrm{L}^{-1}$ dry weight), total lipid and FAME content (\% yield of dry biomass) of Cricosphaera sp. 146-2-9 in monoculture (-C), co-cultured with Fusarium sp. 146-2-F3 (-M), and inoculated in fungal cell-free filtrate (-FS) and marine GPY (-GPY) 


\section{ABBREVIATIONS AND ACRONYMS}

Acetyl-CoA Carboxylase

ACCase

Bacterial supernatant

BS

Carbon dioxide

$\mathrm{CO}_{2}$

Dissolved Inorganic carbon

DIC

Dissolved Oxygen

DO

Dissolved Organic Carbon

DOC

Extracellular polymeric substances

EPS

Fatty acid

FA

Fatty acid methyl esters

FAMEs

Fungal supernatant

FS

Glucose, peptone, yeast-extract

GPY

Water

$\mathrm{H} 2 \mathrm{O}$

Hour

$\mathrm{hr}$

Minute

$\min$

Nitrogen

$\mathrm{N}_{2}$

Sodium Nitrate

$\mathrm{NaNO}_{3}$

Nutrient broth

NB

Non-Taxonomic Groups of Organisms

NTGOs

Optical density

OD

Polyunsaturated fatty acid

PUFA

Rotation per minute

RPM

Yeast Peptone Dextrose

YPD 


\section{CHAPTER 1}

\section{INTRODUCTION}

1.1 Rationale for improving algae biofuel outputs

Procurement and combustion of fossil fuel engenders environmental degradation with climate change and is projected to perpetually increase atmospheric carbon concentrations (IPCC 2014). The increase in atmospheric anthropogenic carbon, pressure on the fossil fuel supply, and growing demand for energy have altogether invigorated interest in biologically-derived renewable fuel resources. One such renewable biofuel is microalgae biomass. Biofuels generated from microalgae are relatively novel; first generation biofuels were derived from sources including starch, sugar and vegetable oil derived from plants. Utilizing crops for fuel production is unfavorable since it competes with and diverts food crops from the global food market and results in the loss of terrestrial biodiversity (Koh et al. 2011). Although second generation biodiesel is an improvement, using non-food crops such as wood and organic waste feedstock in practice is problematic because of the high cost of material and low

energy yield of feedstock. The modern third generation biofuels benefit from oleaginous (lipid or oil-producing) microorganisms favorable for efficient biofuel production.

Using oil-producing algae has advantages over traditional plants and crop residues. Algae are a superior biofuel feedstock as they have high growth rates, 
are competent in unusable water and land (deserts, wastewater, salt water), and can produce of a wide range of fuels and co-products (diesel, jet fuel, hydrocarbons, biogas, ethanol, feed, fertilizer, nutraceuticals and pharmaceuticals, etc) (Hannon et al. 2010). The production of biofuels from algae however faces productivity issues that impede scalability. A central issue governing algal monoculture success is lipid accumulation usually occurs in nutrient depleted conditions. Stress conditions that induce lipid storage in algae, however, inhibit biomass productivity. Moreover, the sustainability and economic cogency of microalgae biofuel production is often questioned in terms of fertilizer requirements (Peccia et al 2013). Fertilizer application represents a significant cost to cultivation and indirect greenhouse gas inputs (Dawson and Hilton 2011). In order for a feasible and sustainable microalgae biofuel production, manipulations of conditions that allow for both rapid lipid and cell growth rate during cultivation are desired (Chisti Y., 2013).

In using algae for biofuel production, one technological aspect has been persistently neglected; that is the effect of accompanying microorganisms on algae growth and lipid accumulation. Integrating co-cultures of microorganisms is becoming a realistic choice for achieving goals of reducing the expenditures associated with cultivation and large-scale production of biofuels. And in using co-cultures of algae and distinct microorganisms, such as nitrogen-fixing cyanobacteria, the use of nitrogenous fertilizer is avoided generating a sustainable algae cultivation system (Mimouni et al. 2012). Improving the 
production of biofuel from algae facilitates the integration of sustainable fuels into the fuel market and helps displace fossil fuel use.

\subsection{Biofuels}

Biofuel is any biologically derived biomass that is converted to produce fuel molecules. Biodiesel, a specific biofuel, is of interest as it can be used directly or blended in the current fuel and automobile infrastructure without modifications such as those required with ethanol or electricity-based technologies. Biodiesel is composed of fatty acid methyl esters (FAMEs). The FAMEs are extracted from triacylglyceride lipid bodies by means of transesterification. The transesterification process is the most common method of producing biodiesel and involves reacting an alcohol with an acid or base catalyst. The alcohol, usually methanol, reacts with the fatty acids chains in forming methyl esters by removing the glycerol backbone of the triacylglycerides (TAGs) (Figure 1). The glycerol waste has many applications including sustainable bioconversion into hydrogen for energy (Sarma et al. 2012).

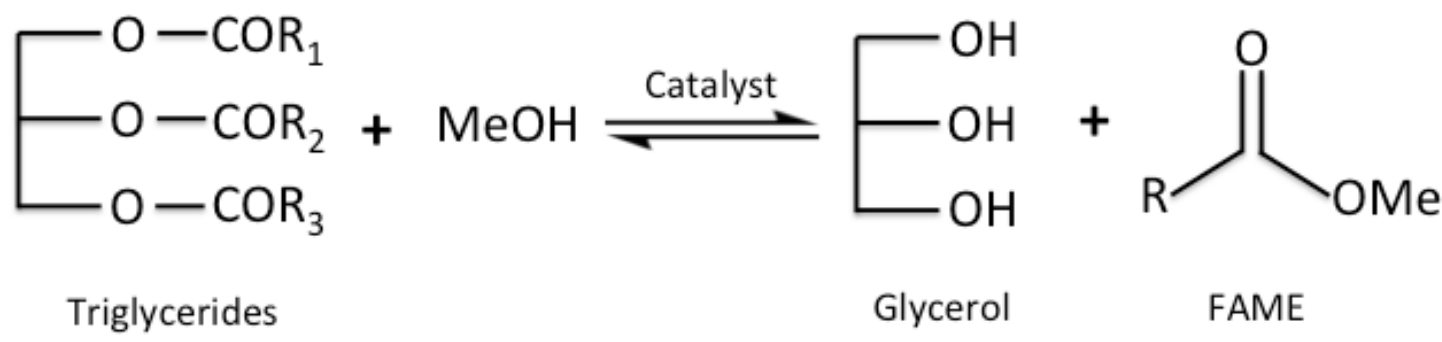

Figure 1 The process of transesterification in manufacturing biodiesel 


\subsection{Biofuel Generations}

Initial attempts at identifying proper sustainable feedstock for the production of biofuels originated in the use of crop plants high in oils. Crops such as soybean, palm, and rapeseed were a few suggested as feedstock in procuring biodiesel. Using crop vegetable oils has displayed great influence in our fuel infrastructure and legislature (U.S. EPA 2010; 2011); nearly 40\% of the U.S. total corn supply has been used for bioethanol production with mandatory mixing requirements into standard fuel (USDA 2015). Using food crops for fuel, however, is unfavorable as it competes with and diverts food crops from the

global food market. Use of the total corn feedstock has increased exponentially over the last decades in response to demands for different applications (USDA 2015; CRS 2013), which consequently resulted in undesired price inflations (Martin 2010). Crops are also notably water, land, and fertilizer intensive and the application of land crops towards mass scale production of fuels may not be sustainable and environmentally sound (CRS 2013). Palms cultivated for biofuels have already resulted in massive land conversions and subsequent biodiversity loss (Koh et al. 2011). Much effort has been made to reduce the use crops for fuel production including establishing regulations that cap the amount of food crops allowed in fuel production (U.S. EPA 2011). In order to overcome the abundant limitations of first generation biofuels, second generation biofuels was developed. 
Second generation biofuels involves the conversion of lignocellulosic feedstock and non-edible agricultural waste into useful fuel. Second generation biofuels was developed in order to support the sustainable use of non-crop feedstock, divert pressures from food crops, and supplement the renewable biofuel mandates (U.S. EPA 2011). In using feedstock rich in lignocellulose, the process of enzymatic hydrolysis can be applied in order to produce ethanol suitable for fuel assimilation. In this platform, non-food crops such as switch grass, Jathropa, and crop residues (husks, stems, leaves) are favored as procurement of these is sustainable. Although second generation biodiesel is an improvement, using non-food crops such as wood and waste feedstock also prove problematic because of the high cost of the enzymatic hydrolysis process, material availability, and low energy yield of feedstock (Carriquiry et al. 2011).

The modern third generation biofuel benefits from oleaginous, lipid or oil producing, microorganisms. The microorganisms often cultivated for fuel purposes include algae. Microalgae have great potential in producing sustainable fuels for several reasons but primarily because microalgae are efficient at generating large amounts of oil per cell (Becker 1994). In comparison to first and second generations biofuels, microalgae generate up to $40-70 \%$ of their biomass in oil, while corn and soy can only provide up to $20 \%$ in oil (Sheehan et al. 1998). Microalgae are also capable of exponential growth cycles, generating biomass and lipids at faster rates than traditional first and second-generation feedstock.

Microalgae are widely distributed, ranging from marine, to freshwater, to saline or brackish water allowing for widespread applications (Schenk 2012). 
Even more remarkable, cultivation of microalgae requires marginal or arable land including deserts and abandoned land and wastewater from sewage effluent (Borowitzka and Moheimani 2010; Maity et al., 2014). Once harvested, microalgae biomass provides multiple fuel types ranging from ethanol and diesel to hydrogen and jet fuel. Lastly, implementing microalgae fuels into current energy practices does not jeopardize the food supply nor does it require a conversion of the current fuel infrastructure. Current vehicle technologies can be used with biodiesel from microalgae, while ethanol and hydrogen fuels require conversion of the establishment to newer capable technologies. Since oil procurement from algae biomass is a promising platform for sustainable fuel production much interest has been invested into optimizing algae strains and the overall process.

\subsection{Algae}

\subsubsection{Algae- definition}

Algae are defined as protists, in the Kingdom Protista, that undergo chlroroxygenic photosynthesis, capable of using chlorophyll a and photons from sunlight in generating oxygen. The informal classification 'algae' is a NonTaxonomic term for Groups of Organisms (NTGOs) used to describe diverse eukaryotic groups of species in different taxonomic branches including, but not limited, to Archaeplastida (red and green algae), Cyanobacteria, and Chromista (Diatoms, brown and golden algae, Dinoflagellates) (Bolton 2016). Algae are ubiquitous organism commonly found in aqueous environments of varying salinity 
(freshwater, brackish, saline) either attached to substrate and bottom dwelling (benthic) or occurring in the water column (planktonic). Algae, whether aquatic or terrestrial, may occur in assemblages such as periphyton or in symbiotic relationships such as coral reefs and lichen (Trexler et al. 2015; Davies et al. 2016; Rikkinen J., 2015). More recently, algae are becoming a notorious consequence of pollution, where toxic harmful algal blooms emerge with detrimental effects to water quality and the health of ecosystems and humans alike (Anderson et al. 2002; Brooks et al. 2016; Trochine et al. 2014).

Algae occur in unicellular and multicellular forms varying in size and degree of complexity. Microalgae are generally of sizes ranging from 1-50 $\mu \mathrm{m}$ while macroalgae can reach several meters in length. Microalgae exist in various forms that have evolved independently among taxa including motile, colonial, coccoid, capsoid, and filamentous. The eukaryotic cell of individual alga is surrounded by a cell wall and may be composed of polysaccharides, proteins, silica, glycoproteins, and sugars depending on the species (Domozych et al. 2012; Okuda 2002). The cell wall encloses a powerhouse that manufactures and stores the lipids crucial to the algae biofuel process.

\subsubsection{Algae biofuel process}

The algae biomass-to-biodiesel process can be artificially summarized into a linear process involving the 1) isolation and identification of a proper algae strain, 2) optimized cultivation of that algae in the proper platform, 3) harvesting and processing of biomass, 4) extraction of the desired oils, and 5) conversion 
and purification of oils into useful products (Figure 2) (Brennan and Owende 2010; Mata 2010).

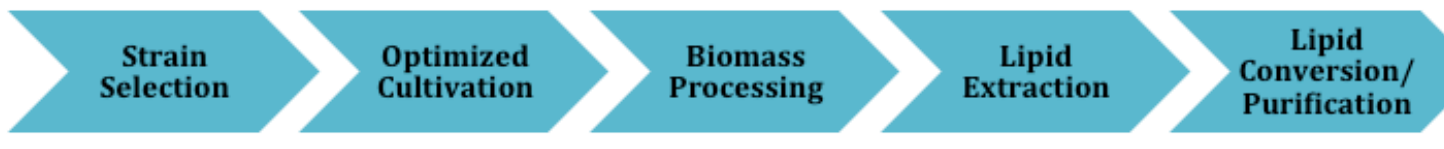

Figure 2 Schematic overview of the algae-based biofuel process

The success of biofuel technologies relies on the selected algal strain and its inherent suitability as a feedstock in facilitating the overall process. There are a multitude of algal characteristics that are preferred for mass cultivation as they directly determine the economic viability and success of algae biodiesel production including lipid profile, productivity, wide environmental adaptations, tolerance to cultivation practices, and ultimately the cell size and morphology of the algal cell (Griffiths and Harrison 2009; Barnard et al 2010).

A fundamental algal characteristic for biodiesel production is the appropriateness of the extracted lipids in relation to quality and quantity. In order to meet biodiesel standards, the chain length and degree of saturation of the triacylglycerides must be suitable to the fuel type as they influence the fuel quality (Islam et al. 2015). Generally, longer chain lengths and highly saturated fatty acids are preferred in the biofuels industry. In contrast, though polyunsaturated fatty acids (PUFAs) may be used as fuel enhancers, they are more commonly used as nutraceutical and pharmaceutical products (Nguyen et al. 2015; Bernstein 2014). There are present trends on the fatty acid (FA) 
distribution within different algal species that can be used as chemotaxonomic markers to define algae taxa (Lang et al. 2011). Research suggests that Chlorophyta is geared towards formation of saturated and monounsaturated compounds with traces of polyunsaturated $\mathrm{C} 16: 0$ and $\mathrm{C} 18: 0$ fatty acids, while Chromalveolates such as Haptophytes (coccolithophores), Stramenopiles (diatoms), and Alveolates (Dinoflagellates) are generally higher in PUFAs. Choosing an algal species with the proper lipid profile is necessary for a successful biofuel technology and having the desired FAs in high abundance is just as imperative.

The proportion of the total algae lipids is also important as it must be composed mainly of triacylglycerides, since other FAs and lipids such as phospholipids and glycolipids may be extracted as well. In quantifying lipid content and determining if an algal strain is suitable for mass production, lipid productivity, the product of biomass productivity $\left(\mathrm{g} \mathrm{L}^{-1}\right.$ day $\left.^{-1}\right)$ and lipid content (\% dry biomass), is the ideal indicator of algae success (Griffiths and Harrison 2009). Lipid productivity reports algae oil produced volumetrically over time as a function of biomass and represents a fine balance between how well a cell grows and its lipid content. Since increasing the oil content of a cell during starvation is not ideal for growth, the overall oil productivity is drastically reduced. High growth rates in conjunction with high lipid content are therefore required to increase yield per unit area and reduce the processing costs per unit of biomass, respectively (Adams et al. 2013; Griffiths and Harrison 2009; Hu et al. 2008). 
Since there are many desirable characteristics for large-scale algae cultivation, it is unlikely that a given strain will be superior in all; including high productivity with appropriate lipid quantity and quality. It is essential to select the species relevant to the method of cultivation and desired product, so choosing algal species with exceptional biomass and lipid productivities from the local environment in which it is cultivated is preferred. Choosing algal species capable of growth in local marginal lands requires adaptations to wide environmental conditions or even extreme environments. A robust alga allows for a facilitated process in wastewaters, changing seasons, and ambient weather with reduced contamination and predation during cultivation.

In cultivation, the available resources and the chosen culture system markedly impacts species choice. In general, algae require a carbon source for photosynthesis and depending on the sources available, the algae in question must assume a specific metabolism pertinent to its application whether it is photoautotrophic, heterotrophic, mixotrophic, or photoheterotrophic (Mata 2010). Cultivation of algae apart from autotrophic systems requires the addition of organic carbon that can result in increased costs and contamination risks (Chen et al. 2011).

Choosing between an open versus close cultivation system also determines the algae species able to grow reliably in a proposed scheme. In an open cultivation system, manmade outdoor ponds or circular raceways are used where algae growth is dependent on natural sunlight and fertilizer, reducing the capital and operating costs (Lee 2001). Though the costs are low, open 
cultivation is restricted to algae that are adapted to competing microorganisms and fluctuating medium salinities and light regimes. In closed cultivation systems, alternatively, a specific medium is used within a sealed photobioreactor that provides artificial light to algae. Photobioreactors may be ideal by providing control over temperature and light irradiance that allows for better success with monocultures, but equipment sterilization is intractable and the energy required in maintaining algae in a closed culture adds to the overall cost of the process.

The next step of the biofuel process is also highly reliant on the algal strain selected. Harvesting of algal cells, or water removal, adds a momentous cost to the process and requires algae species that facilitate harvesting. Harvesting can be done in several ways including centrifugation, flocculation, filtration, and sedimentation (Chen et al. 2011). Harvesting algal species with larger cell sizes and high specific gravity tend to be favored as they are easier and cheaper to harvest and do not require specialized equipment. Morphology of the algae is also critical as natural autoflocculation or aggregation of cells such as colonial or filamentous morphology significantly reduces the energy and equipment required in harvesting and downstream processing (Borowitzka 1997).

Once the algae biomass is harvested and dried the next step of the process involves extraction of the total lipids. In order to extract lipids from algae biomass, several solvents are often used including ethanol, methanol, and hexane with additional assistance from mechanical methods of cell rupture. The techniques of sonication, microwave-assisted extraction, and bead mills are often 
applied in order to the release of intracellular components and facilitate extraction of lipids for downstream purification.

Once the lipids from algae have been extracted, the triacylglycerides must be transesterified into fatty acid alkyl esters in the presence of a catalyst (Figure 1). More recently, the development of supercritical fluid extraction has eliminated the need for catalysts (Warabi et al. 2004). And to date, the separate steps of extraction and transesterification, a two-stage process, have been modified into a single direct transesterification step where the lipids are transesterified from wet biomass (Johnson and Wen 2009; Cao et al. 2013). Eliminating the need to dry the biomass further facilitates the downstream lipid processing and reduces the costs associated with algae biofuels (Haas and Wagner 2011). Besides improving the technologies that facilitate the economical production of fuels from algae, there is considerable interest in developing cultivation schemes that induce superior lipid productivity states within algal strains.

\subsection{Improving algae lipid content}

In the advent of using algae in the production of biofuels, much effort has been employed to increase the lipid content within algae and simultaneously reduce fertilizer use. It has been repeatedly demonstrated that applying nutrient deficient conditions, such as the absence of nitrogen and silicon, typically results in increases in the lipid content of algae. This burst in lipid increase, conversely, often results in decreases in biomass in marine and freshwater species of algae 
across different taxa (Chen et al. 2012; Converti et al. 2009; Li et al. 2008; Rodolfi et al. 2008).

\subsubsection{Cultivation stress and genetic manipulations}

When cultivating algae for biofuel purposes, high lipid productivities are desired for a feasible process (Griffiths and Harrison 2009). Optimization of conditions suitable for both high lipid content and biomass may be contradictory as both rely on opposite culture conditions; algae flourish in nutrient replete conditions while lipid storage occurs in nutrient depleted environments. Other factors that affect lipid accumulation such as salts, temperature, light intensity, and growth phase have also been considered as stress factors in optimizing lipid content but not concurrently with biomass (Hu et al. 2008; Markou and Nerantzis 2013).

A closer look into salt concentration stress in marine media has shown dramatic improvements in lipid content within various algae especially Dunaliella, with unfortunate cutbacks on cell concentration (Salama et al. 2013; Takagi et al. 2006). Temperature and light regimes, though algae may have optimums, can be used to accelerate lipid production when amplified (Xin et al. 2011). At times, the alterations in temperature and light intensity may have implications on the useful types of lipids produced (Chen et al. 2012). Lipid chain lengths with saturation levels and the resultant ratio of PUFA and saturated fatty acid can be dramatically altered in response to different stressors (Xin et al. 2011). Applying 
stress especially nitrogen stress has definite biomass-tradeoffs and the response to this is species-specific (Adams et al. 2013).

To avoid losses in biomass when stress in applied, the method of twostage algae cultivation was proposed. This technique takes advantage of an initial nutrient-supplemented medium to encourage growth and a subsequent cultivation with limited nutrients or stress factors applied to stimulate lipid accretion (Su et al 2010). As these attempts to circumvent biomass loss with lipid gain seem reliable in the lab, however, this technique poses significant operational costs since an additional harvesting step is necessary.

Where species do not meet the requirements nor rely on cultivation manipulations, genetic engineering can be another method of optimizing algal strain characteristics. A central player in the regulation of lipid synthesis, acetylCoA carboxylase (ACCase) has been the focus of much investigation on its enhancement and application in microalgae biofuels. ACCase is the enzyme responsible for the committed formation of malonyl CoA, a precursor to fatty acid formation. In studies where overexpression of ACCase genes was employed in diatoms, an overall increase in ACCase activity is observed, without much influence on lipid yield (Dunahay et al. 1996; Sheenan et al. 1998). Suggested routes to increase lipid synthesis in microalgae are to overexpress genes in pathways associated directly or indirectly with ACCase and lipid synthesis. Targeting TAG assembly genes for overexpression is a promising method for heightened lipid synthesis (Vigeolas et al. 2007). The continued exploration of algal genomes with the improved genetic manipulation toolkits will continue to 
bring about advances in algal metabolic pathways. Genetic engineering, however, is currently restricted to a few algal species and the impeding legislature and environmental risks associated with genetically modified organisms prevent a more expansive application of these algae (Beer et al. 2009; Mata 2010; Sharma et al. 2012).

\subsubsection{Co-Culturing of algae with microorganisms}

Microalgae are abundant in the environment and coexist with a myriad of microorganisms. Symbiotic relationships between algae and other microorganisms play key roles in natural ecosystems and are increasingly becoming of interest to industrial microbiology. The microbial industry is taking advantage of symbiotic relationship by applying them towards the fermented food and water treatment sectors (Silva-Benavides and Torzillo 2012). Application of co-cultures or mixtures of microorganisms have also been suggested in the facilitation of algae biofuels. In this scheme, several different microorganisms have been used as a platform in order to stimulate algal cell growth or production of oils including yeast, fungi, bacteria, and cyanobacteria. The details pertaining to the mechanism that facilitates these microbial relationships, however, remain poorly understood.

i. Co-culturing of algae with yeast

Yeast are unicellular fungi that utilize oxygen in order to process carbohydrates into alcohols and carbon dioxide. As algae require carbon dioxide 
and in turn produce oxygen, the advantageous pairing of yeast and algae is already widely applied in the algae aquaculture research sector (Cai et al. 2007). Pairings of algae such as and Chlorella, Scenedesmus, and Spirulina and yeast have demonstrated increases in biomass and lipid production over monocultures (Xue et al. 2010; Wang et al. 2016). Continued efforts to reduce the cost of the process have been made by using wastewater from industrial refineries or crude glycerol from the biofuel process to cultivate algae co-cultures (Papone et al. 2012; Kitcha and Cheirsilp 2014). In attempts to improve the lipid yield from algae, researchers have proposed the use of oleaginous yeasts such as Rhodotorula glutinis and Trichosporonoides in conjunction with algae (Yen et al 2015). Although lipid and biomass increases have been established, the mechanism by which this occurs still requires elucidation. Co-cultivation of yeast and algae has revealed a rise in algal extracellular polymeric substances (EPS), photosynthetic activity, and dissolved oxygen (DO) and authors have suggested these to explain the heightened relationship between the microorganisms (Wang et al. 2016). The dependency or effect of the microorganisms on EPS or DO, though, have not been clarified.

ii. Co-culturing of algae with bacteria

Phytoplankton and heterotrophic bacteria are among the most abundant microorganisms in aquatic environments (Cole 1982). In both fresh and marine systems, there exists a positive correlation between photosynthesis and microbial activity (Hobbie and Rublee 1977) and this relationship may be a result 
of dissolved organic carbon (DOC) released by senescent algal cells (Bell 1983); as the peak of microbial activity usually occurs at the end of algal blooms (Jones 1972). In the presence of bacteria, algae DOC reach a steady state, whereas in axenic culture the levels persistently increase (Wiebe and Smith 1977). Heterotrophic bacteria can also be associated with living algae cells (Jones 1972); the phycosphere of algae represents a habitat specialized for some bacteria as bacterial populations isolated from water samples dramatically differ from attached populations (Sapp 2007). In the phycosphere, EPS from algae constitutes an organic matter base for bacterial decomposition. Recognized plant phycosphere symbionts such as Rhizobium have already shown growthpromoting effects on algae (Kim et al. 2014).

Conversely, bacteria may also stimulate the growth of algae through mutualism and commensalism (Watanabe et al. 2005). With decomposition, bacteria remineralization can account for much of the assimilated organic compounds, especially phosphorus and nitrogen, in phytoplankton (Axler et al. 1981; Tai et al. 2009). Additionally, where algae demonstrate vitamin auxotrophy, such as cobalamin, thiamine, and biotin requirements (vitamin $B_{12}$, $B_{1}, B_{7}$, respectively), bacteria are able to supplement these through decomposition in co-culture (Croft et al. 2005; 2006).

Attempts in stimulating biomass of algae with symbiotic or mutualistic bacteria include bacteria-algae consortiums and co-cultures (Kim et al. 2014, Kim et al. 2015). Artificially constructed algae consortiums with growth-promoting, attached bacteria have indicated significant stimulation in biomass (Cho et al. 
2015; Higgins and VanderGheynst 2014) with implications on cell morphology and lipid content (de-Bashan et al. 2002). The suggested mechanism for the stimulation in algae growth is an exchange between algal and bacterial cells of DOC for bacteria-synthesized dissolved inorganic carbon (DIC) and sugars. Screening of growth-promoting bacteria on Dunaliella reveals again that algae organic matter recycling by bacteria constitutes a major source of mineralized compounds such as nitrogen (Le Chevanton et al. 2013). To further elucidate the nature of bacteria-algae relationships, the effects of a bacterial-cell free filtrate was tested on algae culture and found that bacterial exudates accounted for much of the observed growth increase in the algae (Park et al. 2008). Though it is understood that bacteria synthesize organic substances that may stimulate algal growth, it remains unclear whether these exudates can stimulate lipid production as well.

iii. Co-culturing of algae with fungi

In using fungi alongside algae in cultivation, research has indicated positive growth correlations. Like most microorganisms, fungi have been especially advantageous in co-culture with algae in treatment of wastewater (Zhou et al. 2012). In optimizing the co-cultivation of microalgae and fungi, like yeast, the use of oleaginous filamentous fungi has also been proposed (Xie et al. 2013). Co-cultures of cyanobacteria, algae and fungi have established synergistic effects on biomass and EPS production (Angelis et al. 2012). Although positive growth is demonstrated, there is a disproportionate focus 
concerned over using filamentous fungi to facilitate the harvesting of algae cells and disregard the lipid content of algae (Zhang and $\mathrm{Hu}$ 2012; Gultom and $\mathrm{Hu}$ 2013; Wrede et al 2014). And when lipid content is considered in a co-cultivation scheme of algae and fungi, the only explanations for the stimulation in biomass and lipid increase is an improved uptake of glucose from the medium and an induced fungal cellulase activity for the consumption of algal cell debris (Xie et al. 2013).

iv. Co-culturing of algae with cyanobacteria

Cyanobacteria are considered an attractive biofuel feedstock as they are photosynthetic and can process endogenous nitrogen in varying forms including ammonium and nitrite (Nozzi et al. 2013). Furthermore, cyanobacteria such as Synechococcus can be applied in the production of ethanol fuels to supplement gasoline (Gao et al. 2012). Cyanobacteria can also be applied in the biodiesel sector when they can concentrate enough fats within the biomass (Karatay and Dönmez 2011). In using this innate nitrogen-producing ability, heterocystous cyanobacteria such as Anabaena have been proposed in order to overcome the nitrogen demand in algae and crop cultivation (Borowitzka and Moheimani 2013). Diazotrophic cyanobacteria are also known to excrete B vitamins that could play a role in supplementation of B-vitamin auxotrophs (Bonnet et al. 2010). In coculturing attempts to reduce nitrogen application, the oleaginous diatom Nitzchia was cultivated with diazotrophic Nodularia with increase in biomass (Lambert 2013). Though diazotrophic cyanobacteria are a potential platform for biofuel 
production, research has failed to indicate if algae biomass and lipid accumulation can both be stimulated in co-culture with algae.

\subsection{Research Significance}

In using algae for production of biofuels it has become essential to choose indigenous algae capable of 1) adapting to local climactic and culturing conditions, 2) producing high lipid yields, and 3) fast cell growth. Cultivation schemes that allow for both high biomass and lipid accumulation are of paramount importance for the economic viability of algal biofuel production. Although altered cultivation factors focused on temperature and nutrients have indicated positive lipid stimulation, biomass is frequently negatively affected. Besides physical manipulations, genetic manipulations of algae, although promising, require transformations with exogenous genetic material and strict cultivation parameters with impending costs (Sharma et al. 2012). In using cocultures to stimulate production of algae, we now understand that bacteria, yeast, fungi, and cyanobacteria may stimulate algae biomass, but the stimulation of lipid accretion and the mechanisms in which biomass and lipid content of algae are stimulated in response to these microorganisms remain largely obscure.

Consequently, my thesis proposes the use of co-cultures of oleaginous, indigenous South Florida, fresh- and saltwater algae with yeast, bacteria, diazotrophic cyanobacteria, and fungi in order to stimulate the production of both biomass and lipids in algae. And in investigating this relationship, the cell-free 
exudates of the microorganisms will be screened in order to possibly elucidate additional mechanisms by which microorganisms trigger increased biomass and lipid productivity of the algal cell in co-culture. 


\section{CHAPTER 2}

\section{MATERIALS AND METHODS}

\subsection{Microorganisms}

\subsubsection{Green algae}

Eight freshwater green microalgae strains (Oocystis sp. 108-5; Chlorella sp. 5-1; Chlorococcum sp. 103-6, 108-4; Coelastrum sp. 46-4; Dactylococcus sp. 64-12; Selenastrum sp. 64-10; Stigeoclonium sp. 64-8) previously indicated to be oleaginous were used in this work (Narendar 2010). In addition, two strains, Chlorella sp. 155-1, Botryococcus sp. 157-1, were procured from the Department of Energy and the University of Texas at Austin, respectively, and used as reference strains. The remaining freshwater and marine strains were isolated from the South Florida region. Sample sites are shown in Figure 2.1. 


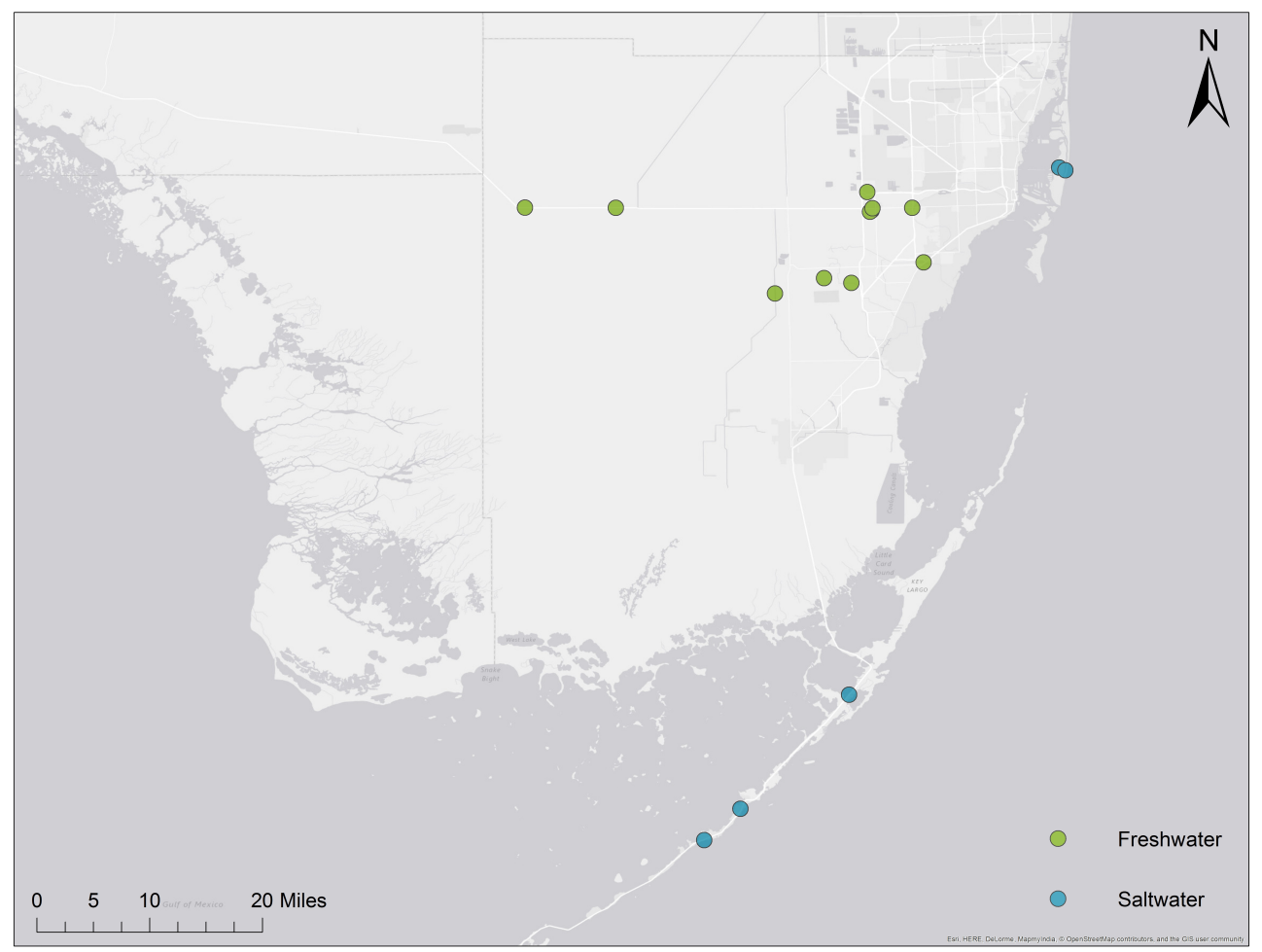

Figure 3 Map of the South Florida region indicating sampling sites

\subsection{2 $\mathrm{N}_{2}$-fixing cyanobacteria}

Nine strains of $\mathrm{N}_{2}$-fixing cyanobacteria were obtained from Dr. Miroslav Gantar's culture collection: Calothrix sp. 38-3, 69-4, 113-9; Nostoc sp. 41-1, 472-1, 47-3, 113-10; Tolypothrix sp. 30-1-4, 33-4. The cultures were maintained on BG11 or on medium without the combined nitrogen BG11 (-N). Cyanobacteria are pictured in below in figure 4 . 


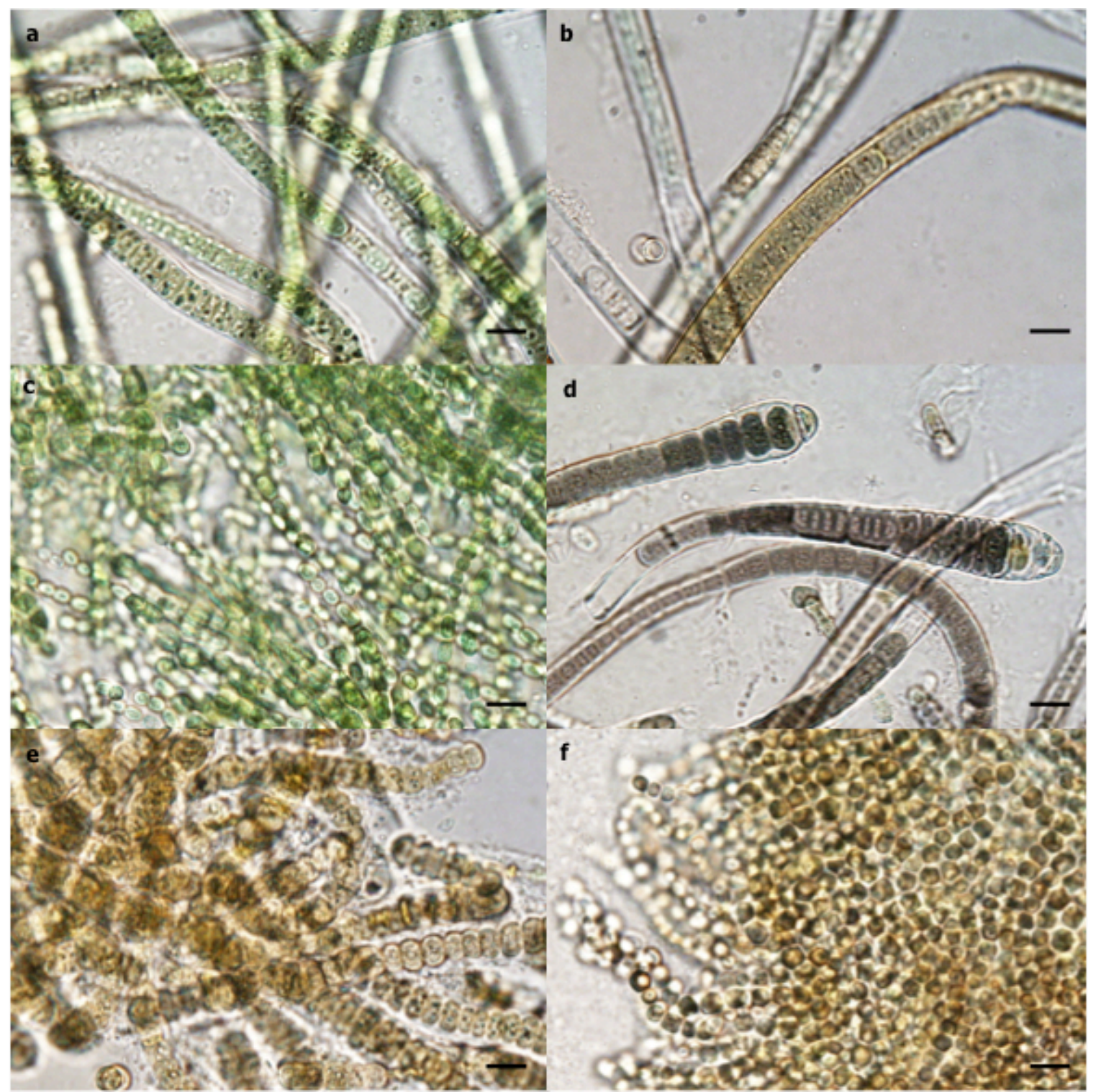

Figure 4 Diazotrophic cyanobacteria used in this research: a) b) Tolypothrix sp. 30-1-4, 33-4, c) Nostoc sp. 47-2-1, d) e) Calothrix sp. 69-4, 113-9, f) Nostoc sp. 113-10. 1000X magnification; scale bar indicates $10 \mu \mathrm{m}$

\subsubsection{Isolation and purification of algae}

Ten freshwater and five saline surface water samples from across the South Florida region were gathered from freshwater canals, lakes, coastline and estuaries as shown in figure 3. Fresh and saline water samples were filtered 
separately through a glass microfiber filter (Whatman GF-C $1.2 \mu \mathrm{m}$ ) and then transferred onto either a BG-11 $(\mathrm{pH}$ 7) and BG-11 Marine $(\mathrm{pH}$ 8) medium plate and incubated at $25^{\circ} \mathrm{C}$ under continuous illumination of $40 \mu \mathrm{mol}$ photons $\mathrm{m}^{-2} \mathrm{~s}^{-1}$ for 2 weeks. Colonies that formed were re-streaked and transferred into $2 \mathrm{ml}$ of sterile medium.

For screening purposes, all microalgae isolates used in this research were rendered axenic by methods of atomized cell spraying and use of antibiotics. One $\mathrm{ml}$ of each culture was pipetted into $1.5 \mathrm{ml}$ eppendorf tube, centrifuged, and washed with sterile medium three times. Cell suspensions were aseptically sprayed onto BG-11 (Marine where appropriate) agar plates amended with antibiotic mix of azithromycin, cycloserin, kanamycin, and streptomycin at $100 \mu \mathrm{g}$ $\mathrm{ml}^{-1}$ and incubated at $25^{\circ} \mathrm{C}$ under continuous light $\left(40 \mu \mathrm{mol}\right.$ photons $\left.\mathrm{m}^{-2} \mathrm{~s}^{-1}\right)$ for 2 weeks (Su et al. 2007). Absence of contamination was verified by plating treated microalgae cultures onto Nutrient Agar (Difco, USA) and incubating in dark at $25^{\circ} \mathrm{C}$ for 1 week.

2.1.4 Isolation of yeast, fungi, and bacteria

Yeast cultures were isolated either from the 10 freshwater samples procured from the sites located in the South Florida region or from xenic microalgae strains from the culture collection. Acquiring yeasts from microalgae cultures involved plating and streaking $100 \mu \mathrm{L}$ of xenic microalgae culture onto YPD (Yeast, Peptone, Dextrose) plates and incubating at $25^{\circ} \mathrm{C}$ for 2 weeks. 
Freshwater samples from the South Florida region were processed for yeast by filtration through a Whatman $0.2 \mu \mathrm{m}$ filter and incubated onto YPD plate in the dark at $25^{\circ} \mathrm{C}$ for 1 week. Distinct yeast colonies were re-streaked, separated, and maintained on YPD slants.

Marine fungi were isolated from the five South Florida saline samples. Water samples were filtered through GC $0.45 \mu \mathrm{m}$ microfiber filters and transferred to GPY (1g Glucose; 0.5g Peptone; 0.1g Yeast extract; 15g Agar; $1 \mathrm{~L}$ seawater) plates and incubated in the dark at $25^{\circ} \mathrm{C}$ for 1 week (Kossuga et al. 2012). Individual filamentous formations were re-streaked, isolated and transferred into tubes with sterile Marine GPY medium. Where needed, filamentous fungi that were contaminated with yeast were plated and submerged in sterile deionized water in order to release spores. Spore suspension was then pipetted into a $15 \mathrm{ml}$ Falcon tube and centrifuged at $2000 \mathrm{~g}$ for 10 minutes, and washed three times with first dilute bleach (50\%) and then sterile deionized water. Spores were then plated onto GPY plates and axenic cultures were separated.

Bacteria were isolated from the freshwater microalgae isolated and the xenic microalgae from the Dr. Miroslav Gantar's culture collection. A total of 40 freshwater bacteria were used in this research and maintained on Nutrient Agar and grown in Nutrient broth for propagation (Difco, USA). 


\subsubsection{Identification of microorganisms}

\subsubsection{Identification of algae Isolates}

Genus-level taxonomic identification was based on morphology and was carried out using microalgae identification guides (Bellinger 2010; Werh 2003). Microscopic visualization and recording of algal isolates was performed using an Olympus BX51 microscope equipped with an Olympus DP70 camera and DP controller software, and were processed using Olympus DP manager software.

\subsubsection{Identification of bacterial and fungal Isolates}

The fungal and bacterial isolates that indicated improved biomass and lipid productivity within the designated microalgae were selected for identification using Biolog FF and GEN III micro plates (Biolog, Hayward, CA), respectively. For each isolate, $100-\mu l$ of the cell suspension was inoculated into each well of the Biolog Microplate according to manufacturer's protocol. Plates were incubated at $26^{\circ} \mathrm{C}$ for fungi and $33^{\circ} \mathrm{C}$ for bacteria for $24 \mathrm{hrs}$ and read in the GENIII MicroStation ${ }^{\mathrm{TM}}$ system semi-automated reader and the results were interpreted with the Biolog MicroLog ${ }^{\mathrm{TM}} 3$ Software (Version 6.1) database and software (Biolog, Hayward, CA). Results that indicated similarity and Dis values of 0.5 and 2 and above were accepted as positive identifications. 


\subsection{Screening of Microalgae}

\subsubsection{Growth and Biomass Productivity of Freshwater Microalgae}

Microalgae seed cultures were maintained in $75 \mathrm{ml}$ of BG11 medium in $125 \mathrm{ml}$ flasks with agitation at $120 \mathrm{rpm}$. Flasks were kept in an orbital shaker incubator (Lab-Line) at $150 \mathrm{rpm}$ and $25^{\circ} \mathrm{C}$ under continuous light $(50 \mu \mathrm{mol}$ photons $\mathrm{m}^{-2} \mathrm{~s}^{-1}$ ). Twenty-two exponential-phase microalgae were inoculated into $35 \mathrm{ml}$ of BG 11 medium in $50 \mathrm{ml}$ flasks $\left(0.1 \mathrm{OD}_{600}\right)$ with three replicates each. Flasks were incubated with continuous lighting of $80 \mu \mathrm{mol}$ photons $\mathrm{m}^{-2} \mathrm{~s}^{-1}$ at $25^{\circ} \mathrm{C}$ on a shaker at $120 \mathrm{rpm}$. Growth was monitored spectrophotometrically as absorbance at $600 \mathrm{~nm}$ every $5^{\text {th }}$ day for 30 days. Biomass productivity, based on

units of grams per liter per day $\left(\mathrm{g} \mathrm{l}^{-1}\right.$ day $\left.^{-1}\right)$, was assessed gravimetrically by filtering a $1 \mathrm{ml}$ aliquot from each of the three replicates through a Whatman GF-C $(1.2 \mu \mathrm{m})$ glass microfiber filter every 5 days for 30 days. Filter disks were dried in the oven to constant weight $\left(50^{\circ} \mathrm{C}\right)$.

\subsubsection{Lipid productivity of Freshwater Microalgae}

Lipid content of the 22 strains of microalgae is determined by homogenizing a $1 \mathrm{ml}$ aliquot of each replicate and diluting to $0.1\left(\mathrm{OD}_{600}\right)$ for staining with Nile Red based on previous methods (Chen et al. 2009). $1 \mathrm{ml}$ of the cell suspension is pipetted into an eppendorf tube, DMSO (99.1\% Sigma, USA) is added at $20 \%$ final concentration and vortexed. $100 \mu \mathrm{l}$ of the cell suspension is pipetted into a 96-well plate for autofluorescence and absorbance determinations. To the remaining $1100 \mu \mathrm{l}$ of the cell suspension, $1 \mu \mathrm{l}$ of Nile Red 
solution ( $1 \mathrm{mg} / 25 \mathrm{ml}(\mathrm{w} / \mathrm{v})$ acetone) was added for a final staining concentration \pm $1 \mu \mathrm{gml}^{-1}$ and vortexed. After incubation, each tube is vortexed and $100 \mu \mathrm{l}$ of cell suspension is then transferred into a 96-well plate. Absorbance and fluorescence readings were carried out using a plate reader (Biotek; Synergy HT) and accompanying Gen5 software (2.0). Excitation and emission wavelengths of $530 \mathrm{~nm}$ and $575 \mathrm{~nm}$ were used as standards for neutral lipids. Relative fluorescence intensity of Nile red was calculated after subtraction of microalgae autofluorescence and the fluorescence of Nile red in medium (Lee et al. 1998). The fluorescence values are then normalized by dividing with the absorbance values $\left(\mathrm{OD}_{600}\right)$. To translate the amount of fluorescence present in $100 \mu \mathrm{l}$ of cell suspension into lipid content, a standard curve using the biofuel lipid standard Triolein was used.

Triolein lipid standard curves are constructed based on the modified methods of Priscu et al, (1990). A 1.001g aliquot of pure Triolein (99.9\%, Sigma Aldrich, USA) is dissolved into an equal volume of chloroform and then diluted into $100 \mathrm{ml}$ of ethanol to create a primary stock solution. Diluting appropriate amounts of the primary stock solution into ethanol created intermediate stocks of $0.1,0.2,0.5,1.0$, and $2.0 \mathrm{mg} \mathrm{ml}^{-1}$ solutions. $100 \mu \mathrm{l}$ of each intermediate stocks solution is than pipetted into $10 \mathrm{ml}$ of deionized water to create working standards with concentrations of $1.0,2.0,5.0,10.0$, and $20.0 \mu \mathrm{g} \mathrm{ml}^{-1}$. A $1 \mathrm{ml}$ aliquot of each working standard is then transferred into a $1.5 \mathrm{ml}$ eppendorf tube and $10 \mu \mathrm{L}$ of Nile Red is added for a final staining concentration of $1 \mu \mathrm{g} \mathrm{ml}^{-1}$. After a 10 min dark incubation, $100 \mu \mathrm{L}$ of each sample is transferred to a 96-well plate and 
procedures follow as previously described. The blank includes chloroform, ethanol, deionized water, and Nile Red. Vortexing for 1 minute before pipetting is vital to keep a uniform distribution of lipid micelles within the solution. A standard curve was constructed with a $R^{2}=0.99399\left(y=4.4836 \mathrm{e}^{0.8335 x}\right)$. Fluorescence values are then transformed into lipid quantities. Lipid productivity of each strain is then calculated using units of $\mathrm{mg} \mathrm{I}^{-1}$ day $^{-1}$.

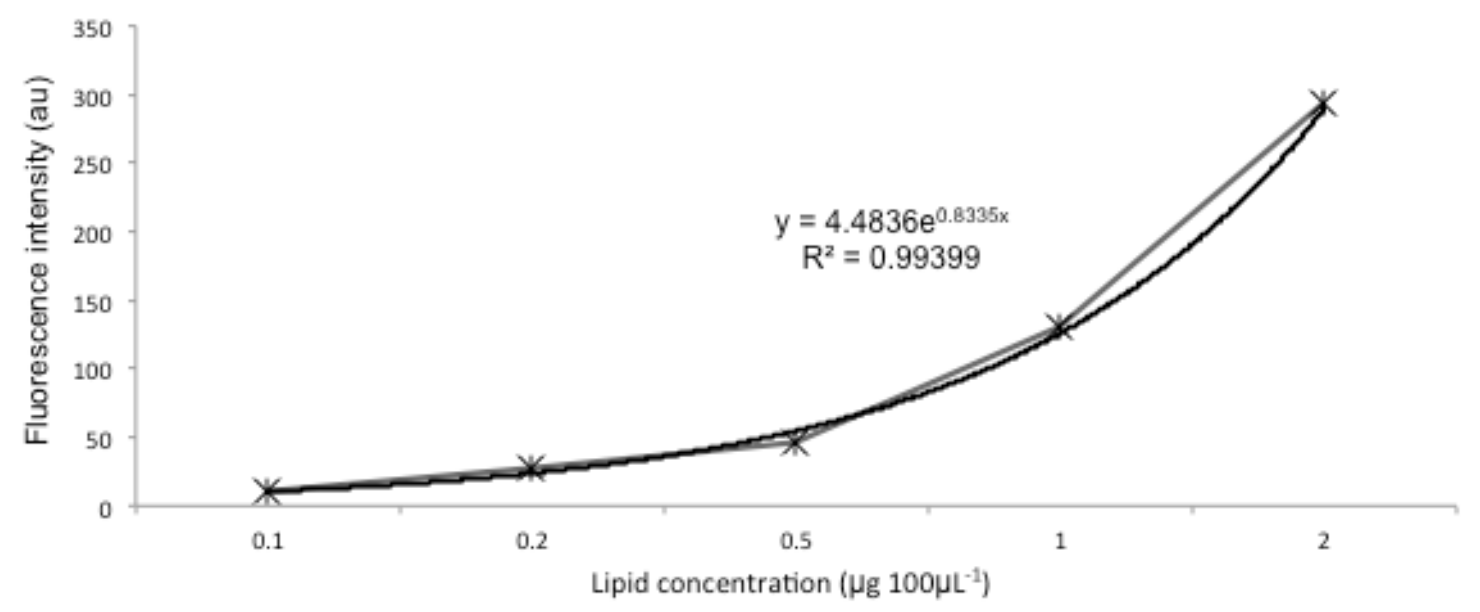

Figure 5 Triolein lipid standard curve used to quantify lipid concentration within algal cell culture

\subsubsection{Biomass and Lipid Productivity of Marine Microalgae}

To determine marine microalgae with high lipid and biomass productivity, individual axenic strains are inoculated in triplicates into $30 \mathrm{ml}$ of BG-11 Marine medium in $50 \mathrm{ml}$ flasks incubated on a shaker $(130 \mathrm{rpm})$ at $25^{\circ} \mathrm{C}$, under continuous fluorescent lighting $\left(80 \mu \mathrm{mol}\right.$ photons $\left.\mathrm{m}^{2} \mathrm{~s}^{1}\right)$. Biomass productivity is assessed gravimetrically by filtering a $1 \mathrm{ml}$ aliquot from each three replicate 
through a Whatman GF-C $(1.2 \mu \mathrm{m})$ glass microfiber filter every 5 days for 30 days. Filter disks are dried in the oven to constant weight $\left(50^{\circ} \mathrm{C}\right)$. Lipid productivity is then assessed using the Nile Red method as previously described (section 2.2.2).

2.3 Screening for high biomass and lipid production in co-cultures

2.3.1 Growth and lipid content of freshwater microalgae and yeast

Prior to co-culturing experiments, yeast strains are inoculated into $3 \mathrm{ml}$ sterile YPD broth $(\mathrm{pH} 6.2)$ in $15 \mathrm{ml}$ autoclaveable/centrifugable Falcon tubes, covered with foil, and placed on a shaker at $150 \mathrm{rpm}$ at $25^{\circ} \mathrm{C}$ for 1 week. Exponential phase microalgae cultures are inoculated into 24-well plates with $0.02 \mathrm{OD}_{600}$ in triplicates. Falcon tubes with yeast are centrifuged at $2000 \mathrm{~g}$ (Beckman Coulter GRP) for 15 minutes, supernatant removed, and pellets are washed with sterile BG-11 medium. Yeast pellets are then diluted with medium until $\mathrm{OD}_{600}$ reaches 0.05 . A $100-\mu \mathrm{l}$ aliquot of the individual yeast solution is pipetted into 24-well plates containing the algal culture. Plates are incubated under continuous lighting of $50 \mu \mathrm{mol}$ photons $\mathrm{m}^{-2} \mathrm{~s}^{-1}$ at $25^{\circ} \mathrm{C}$ for two weeks. Growth and lipid content are assessed by sampling a $1 \mathrm{ml}$ aliquot for $\mathrm{OD}_{600}$ and Nile Red fluorescence, respectively, for two weeks. 
2.3.2 Growth and lipid content of freshwater microalgae with bacteria

Thirty-nine bacterial isolates are inoculated into $3 \mathrm{ml}$ sterile Nutrient Broth in $15 \mathrm{ml}$ autoclaveable/centrifugable Falcon tube $(\mathrm{pH} 6.8)$, covered with foil, and placed on a shaker at $150 \mathrm{rpm}$ at $25^{\circ} \mathrm{C}$ for 1 week. Falcon tubes with bacteria are then centrifuged at $2000 \mathrm{~g}$ (Beckman Coulter GRP) for 15 minutes, supernatant removed, and pellets are washed with sterile BG-11 medium. Bacteria pellets are then diluted with $\mathrm{BG}-11$ medium until $\mathrm{OD}_{600}$ reaches 0.02 . Exponential phase of Coelastrum sp. $46-4$ cultures inoculated into 24 -well plates with $0.02 \mathrm{OD}_{600}$ in triplicates. A $100-\mu l$ aliquot of individual bacterial solution is pipetted into the 24well plate containing the algae. Plates were incubated under continuous lighting of $50 \mu \mathrm{mol}$ photons $\mathrm{m}^{-2} \mathrm{~s}^{-1}$ at $25^{\circ} \mathrm{C}$ for two weeks. Growth and lipid content are assessed by sampling a $1 \mathrm{ml}$ aliquot for $\mathrm{OD}_{600}$ and Nile Red fluorescence, respectively, for two weeks.

2.3.3 Growth and lipid content of freshwater microalgae and $\mathrm{N}_{2}$-fixing cyanobacteria

Nine strains of cyanobacteria were co-cultured with one oleaginous strain of green microalga (Coelastrum sp. 46-4). Combinations of microalgae with individual cyanobacterium were grown in the presence $(\mathrm{N}+)$ and the absence $(\mathrm{N}-)$ of sodium nitrate $\left(\mathrm{NaNO}_{3}\right)$. Cultures were inoculated in triplicates in $150 \mathrm{ml}$ flasks and incubated on a shaker $(130 \mathrm{rpm})$ at $25-27^{\circ} \mathrm{C}$, under continuous fluorescent lighting $\left(80 \mu \mathrm{mol}\right.$ photons $\left.\mathrm{m}^{2} \mathrm{~s}^{1}\right)$. Biomass and lipid productivity were determined 
by measuring absorbance at $600 \mathrm{~nm}$ and by staining cells with Nile Red and quantifying the fluorescence at 530/575 nm, respectively. Biomass and lipid content were measured every $5^{\text {th }}$ day for 15 days.

\subsubsection{Growth and lipid content of marine microalgae and fungi}

Fungal strains are inoculated into $3 \mathrm{ml}$ sterile GPY Marine broth $(\mathrm{pH} 8.0)$ in $15 \mathrm{ml}$ autoclaveable/centrifugable Falcon tubes and placed on a shaker at $150 \mathrm{rpm}$ at $25^{\circ} \mathrm{C}$ for 1 week in the dark. Exponential phase microalgae cultures are inoculated into $30 \mathrm{ml} \mathrm{BG}-11$ Marine medium in $50 \mathrm{ml}$ flasks with $0.02 \mathrm{OD}_{600}$ in triplicates. Falcon tubes with fungi were centrifuged at 2000g (Beckman Coulter GRP) for 15 minutes, supernatant removed, and pellets are washed with sterile medium three times. Fungal pellets are then aseptically homogenized with a hand-held homogenizer and then diluted with BG-11 Marine medium until $\mathrm{OD}_{600}$ reached 0.05 . A $100-\mu \mathrm{l}$ aliquot of the fungal solution is pipetted into the flasks containing the algal cultures. Flasks are then incubated under continuous lighting of $50 \mu \mathrm{mol}$ photons $\mathrm{m}^{2} \mathrm{~s}^{-1}$ at $25^{\circ} \mathrm{C}$ and shaken (125 rpm) for two weeks. Growth and lipid content are assessed by measuring the biomass and Nile Red fluorescence, respectively, every $5^{\text {th }}$ day for 25 days.

2.4 Biomass and lipid productivity of co-cultures

One freshwater algal (Coelastrum sp. 46-4) strain that demonstrated superior lipid productivities with accompanying microorganism was selected for 
further lipid quantifications. All combinations of microalgae with bacteria, fungi, and cyanobacteria are first evaluated on a small scale and then on a greater scale to accumulate biomass for lipid gravimetric analysis. The marine alga Cricosphaera sp. 146-2-9 was selected and screened with 3 different strains of marine fungi.

2.4.1 Co-cultivation vs. bacterial and fungal-cell free filtrate

In order to determine whether accompanying microorganisms affect the growth and/or lipid accumulation in algal cultures the following experiment was performed. The accompanying microorganisms (bacteria and fungi) were grown in $75 \mathrm{ml}$ of the respective mediums: Nutrient broth and marine GPY and incubated in the dark, on a shaker $(130 \mathrm{rpm})$ at $25^{\circ} \mathrm{C}$ for one week. Cultures are then harvested by centrifuging in sterile $50 \mathrm{ml}$ glass centrifuge tubes and centrifuged at $2000 \mathrm{~g}$. Cell pellets are then washed three times with BG-11 for bacteria, and BG-11 marine for fungi and diluted until $\mathrm{OD}_{600}$ reaches 0.05 . Supernatants are then sequentially filtered first through a Whatman GF-C 1.2 $\mu \mathrm{m}$, then a $0.45 \mu \mathrm{m}$ syringe filter, and finally a $0.2 \mu \mathrm{m}$ syringe filter. Microalga (freshwater and marine) is then inoculated in quadruplets with initial $\mathrm{OD}_{600} 0.02$ into $30 \mathrm{ml}$ of appropriate medium, (1) alone, (2) medium composed of 1:1 (v/v) BG-11 (marine) and filtered supernatant, (3) medium composed of 1:1 (v/v) BG11 (marine) and sterile microorganism broth (NB, BG-11 Marine GPY), and (4) medium inoculated with $100 \mu \mathrm{l}$ of microorganism diluted pellet. Flasks are then 
incubated on a shaker $(130 \mathrm{rpm})$ at $25^{\circ} \mathrm{C}$, under continuous fluorescent lighting $\left(80 \mu \mathrm{mol}\right.$ photons $\left.\mathrm{m}^{2} \mathrm{~s}^{1}\right)$. Biomass and lipid productivities are assessed as previously described (section 2.2 .2 ) every $5^{\text {th }}$ day for 20 days.

\subsubsection{Gravimetric analysis of lipid content}

In order to estimate lipid content in the biomass by gravimetric method, the accompanying microorganisms (bacteria and fungi) were grown in 2- $1.5 \mathrm{~L}$ of respective medium (nutrient broth and GPY) and incubated in the dark at $25^{\circ} \mathrm{C}$ for 1 week. Microbial cultures are harvested by centrifuging first in sterile $50 \mathrm{ml}$ glass centrifuge tubes at $2000 \mathrm{~g}$ (Beckman Coulter GRP). Cell pellets are then washed three times with BG-11 for bacteria, and BG-11 marine for fungi and diluted until $\mathrm{OD}_{600}$ reaches 0.05 . Supernatants are then pooled and transferred into Nalgene centrifuge bottles and centrifuged for $30 \mathrm{~min}$ at $4000 \mathrm{~g}$ (Beckman Coulter, Avanti J-20 XP). Supernatants are then sequentially vacuum-filtered, first through a Whatman GF-C $1.2 \mu \mathrm{m}$, GF-B $1.0 \mu \mathrm{m}$, then finally a Corning 0.22 $\mu \mathrm{m}$ bottle-top vacuum filter. Freshwater microalgae is then inoculated in duplicates with initial $\mathrm{OD}_{600} 0.02$ into 2-3 L of appropriate medium: (1) BG11, (2) medium composed of 1:1 (v/v) BG-11 and microbial cell-free supernatant, (3) BG-11 inoculated with $1 \mathrm{ml}$ of microorganism diluted pellet mixture, and 4) medium composed of 1:1 (v/v) BG-11 and nutrient broth. The nutrient broth and BG-11 were substituted with GPY and BG-11 marine for the saltwater algae. Then the cultures were incubated under continuous lighting of $50 \mu \mathrm{mol}$ photons $\mathrm{m}^{-2} \mathrm{~s}^{-1}$ at $25^{\circ} \mathrm{C}$ and aerated with sterile air. 
Co-cultures with cyanobacteria involve inoculating microalgae alone and in combinations with individual cyanobacterium in 3 liters of BG-11 medium with and without $\mathrm{NaNO}_{3}$. Cultures are incubated at $25^{\circ} \mathrm{C}$ under continuous fluorescent lighting $\left(80 \mu \mathrm{mol}\right.$ photons $\left.\mathrm{m}^{2} \mathrm{~s}^{1}\right)$ aerated with sterile air.

All large-scale co-culture experiments are harvested on day 15 by centrifugation at $3000 \mathrm{~g}$ (Beckman Coulter, Avanti J-20 XP). Biomass is then transferred into pre-weighted $50 \mathrm{ml}$ Falcon tubes, and freeze-dried for further productivity analysis.

Once the dry biomass of co-cultures was obtained, lipids were extracted as follows: $0.250 \mathrm{~g}$ of freeze-dried microalgae biomass was placed in a glass centrifuge tube and $22.5 \mathrm{ml}$ of chloroform: methanol $(2: 1 \mathrm{v} / \mathrm{v})$ was added and placed on a rotary shaker for $20 \mathrm{hrs}$ in order to extract neutral lipids. Tubes were sonicated for 10 minutes in order to facilitate cell rupture. After incubation, distilled water was then added to a final volume of chloroform: methanol: water mixture 2:1:1 for phase separation. Glass tubes were centrifuged at $2000 \mathrm{~g}$ for 15 minutes to separate the organic and aqueous phase. The aqueous phase is discarded and the organic phase is transferred to a pre-weighted glass petri dish. The biomass is extracted two more times and the pooled organic phases are collected onto the petri dish. Samples are then air dried in a fume hood under sterile air. Data are the means of three separate replicate extractions (D'Oca et al. 2011). 


\subsubsection{Transesterification of Lipids}

To yield fatty acid methyl esters (FAMEs), lipid extracts from co-culture experiments were transesterified using modified techniques adopted from (D'oca et al. 2011). Entire lipid extract of each sample from section 2.4.2 was placed in a glass vial and mixed with $10 \%$ sulfuric acid and chloroform/methanol (30:1). The reaction was carried out under constant stirring at $60 \mathrm{C}$ for 4 hours. Once cooled to room temperature, reaction mixture was transferred to glass centrifuge vials, and centrifuged for $30 \mathrm{~min}$. Aqueous phase was discarded and the organic phase was transferred to a pre-weighted glass vial.

\subsection{Statistical Analysis}

Statistical analysis was carried out using SPSS software (version 22.0). A one-way repeated measures ANOVA was used to determine any differences between the treatment means of the small-scale experiments involving freshwater and marine algae in co-culture with bacteria, cyanobacteria, and fungi, respectively over the two-week period. The ANOVA was used to test for any differences in the mean biomass and lipid accumulations of monocultures, co-cultures, and cultures inoculated in sterile microorganism medium and microorganism cell-free filtrate. Tukey post-hoc analysis was used to determine where the significant differences occurred in the mono- versus co-cultures. 


\section{CHAPTER 3}

\section{RESULTS}

3.1 Isolation and identification of microorganisms

3.1.1 Freshwater and marine algae

From the 10 South Florida freshwater samples (Figure 3), a total of 14 microalgae species were isolated, cultivated, and screened for biomass and lipid productivity along with the remaining strains from the culture collection for this research (Figure 6). The microalgae strains represented 11 genera within Chlorophyceae (Table 1).

Table 1 Chlorophyceae algae used in this research

\begin{tabular}{ccc}
\hline Microalgae Genus & Strain Identification \# & Origin \\
\hline Dictyococccus & $64-12$ & CC \\
Chlorococcum & $142-5-2,143-1,143-5,143-$ & FL (5), CC \\
Coelastrum & $6,145-7,103-6$ & CC (3), FL \\
Dictyochloropsis & $5-1,46-6,108-4,143-5$ & FL \\
Stigeoclonium & $145-7$ & CC \\
Scenedesmus & $64-8$ & FL \\
Chlorella & $143-4,145-2,145-3,148-5-2$ & DOE, FL \\
Botryococcus & $155-1,150-1-1$ & UTEX \\
Characium & $157-1$ & FL \\
Oocystis & $152-3$ & CC, FL \\
Selenastrum & $108-5,148-1-1$ & CC \\
\hline Origin (CC- Culture Collection, FL-species isolated for this research)
\end{tabular}




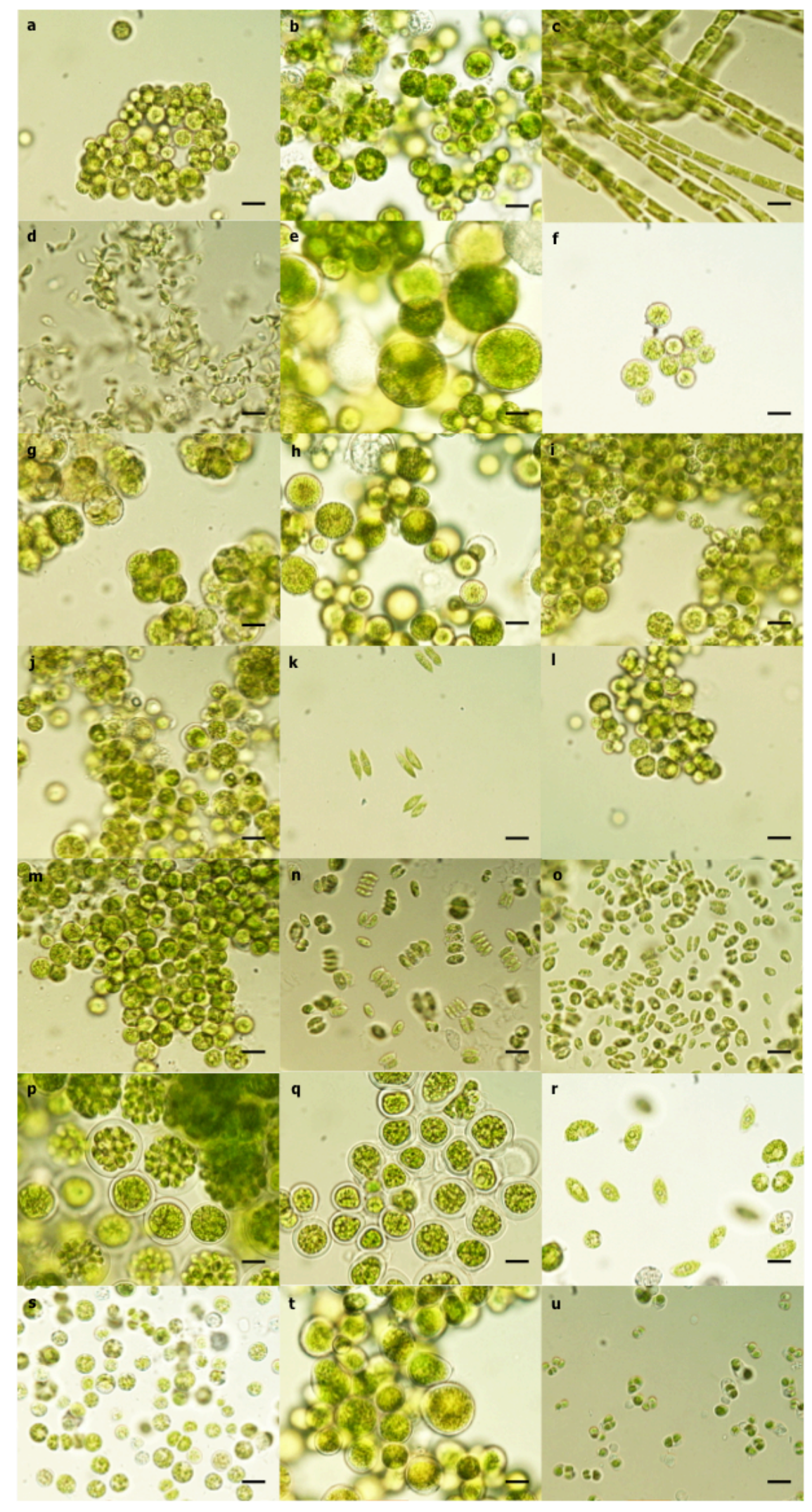


Figure 6 Freshwater algae used in this research; a) b) Coelastrum sp. 5-1, 46-4, c) Stigeoclonium sp. 64-8, d) Selenastrum sp. 64-10, e) Dictyococcus sp. 64-12, f) Chlorococcum sp. 103-6, g) Oocystis sp. 108-5, h) i) Chlorococcum sp. 142-52, 143-1, j) Coelastrum sp. 143-5, k) Scenedesmus sp. 143-4, I) Chlorococcum sp. 143-6, m) Coelastrum sp. 108-4, n) o) Scenedesmus sp. 145-2, 145-3, p) Dictyochloropsis sp. 145-7, q) Oocystis sp. 148-1-1, r) Scenedesmus sp. 148-52, s) Chlorella sp. 150-1-1, t) Characium sp. 152-3, u) Chlorella sp. 155-1. 1000X magnification; Scale bar indicates $10 \mu \mathrm{m}$

From the five saline water samples in Figure 3, nine marine algae strains were isolated and used in this research (Figure 7). The marine algae represent three different classes including Chlorophyceae, Eustigmaphyceae, and Prymnesiophyceae (Table 2).

Table 2 Isolated marine algae (BG11 marine)

\begin{tabular}{ccc}
\hline Class & Genus & Strain Identification \# \\
\hline Chlorophyceae & Tetraselmis & $146-2-1$ \\
Chlorophyceae & Chlorococcum & $146-2-6$ \\
Eustigmatophyceae & Vischeria & $146-2-8$ \\
Prymnesiophyceae & Cricosphaera & $146-2-9$ \\
Chlorophyceae & Chlamidomonas & $146-2-10$ \\
Prymnesiophyceae & Chrysotila & $146-2-11$ \\
Eustigmatophyceae & Eustigmatos & $146-2-14$ \\
Chlorophyceae & Chlorella & $146-2-15$ \\
Eustigmatophyceae & Vischeria & $146-2-16$ \\
\hline
\end{tabular}




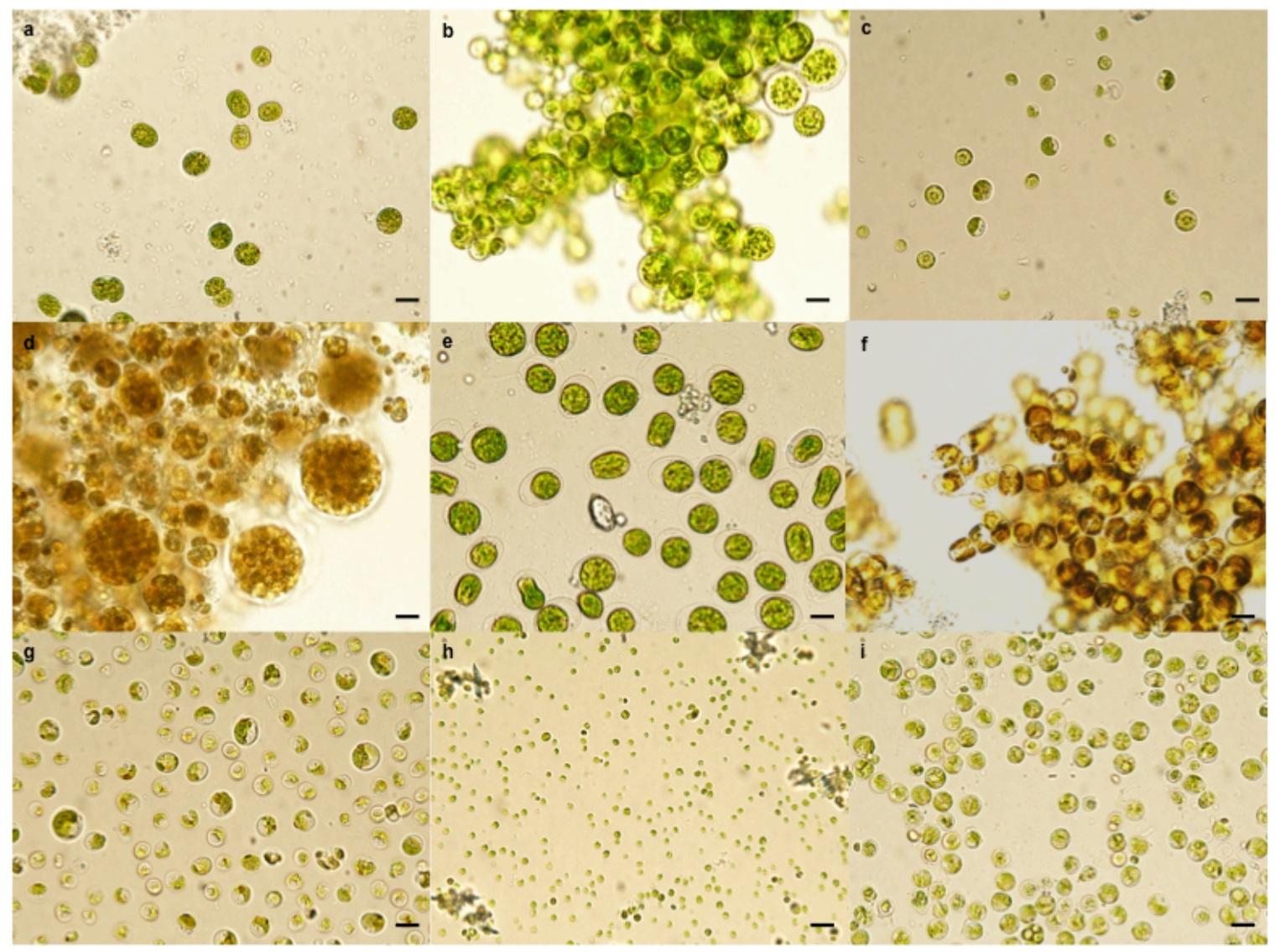

Figure 7 Marine algae isolated for this research; a) Tetraselmis sp. 146-2-1, b) Chlorococcum sp. 146-2-6, c) Vischeria sp. 146-2-8, d) Cricosphaera sp. 146-29, e) Chlamidomonas sp. 146-2-10, f) Chrysotila sp. 146-2-11, g) Eustigmatos sp. 146-2-14, h) Chlorella sp. 146-2-15, i) Vischeria sp. 146-2-16. 1000X magnification; Scale bar indicates $10 \mu \mathrm{m}$

\subsubsection{Bacteria, Yeast, and Fungi}

A total of 38 yeast, 37 bacteria, and 3 fungal species were isolated from both the freshwater samples and xenic microalgae from the culture collection, and the saline samples, respectively. The bacterial isolate 21-9-3-2 and fungal isolate 146-2-F3 were both selected for stimulation of the biomass and lipid productivity of Coelastrum sp. 46-4 and Cricosphaera sp. 146-2-9, respectively. 
Biolog database similarity index revealed a positive identification for bacterial isolate 21-9-3-2 as Pseudomonas stutzeri, a motile, gram negative, denitrifying bacteria with a probability of $57.2 \%$ (Lalucat et al. 2006). Fungal isolate 146-2-F3 was identified as Fusarium sp., a filamentous fungi belonging to the Ascomycota phylum of Fungi.

\subsection{Freshwater algae biomass and lipid productivity}

The results from the studies involving screening of 22 strains of green algae showed wide variation among strains in biomass and lipid accumulation capabilities (Table 3 ). The average biomass content for all 22 algal strains cultivated for 20 days was $0.27 \mathrm{~g} \mathrm{~L}^{-1}$ while the average biomass productivity was $0.025 \mathrm{mg} \mathrm{L}^{-1} \mathrm{day}^{-1}$. The highest biomass accumulation or all strains occurred on day 20. The control strains Botryococcus sp. 157-1 and Chlorella sp. 155-1 were observed to have very similar biomass accumulation throughout the experiment. Over the 20-day culturing period, the control strains did not produce biomass above $0.37 \mathrm{~g} \mathrm{~L}^{-1}$. On day 20 , the highest biomass accumulation was observed in strains Coelastrum sp. 46-4, Dictyococcus sp. 64-12, and Chlorococcum sp. 1425-2 with resulting biomass of $0.83,0.57$, and $0.57 \mathrm{gL}^{-1}$, respectively. Over the 20 day cultivation, the highest average biomass productivity occurred with Coelastrum sp. $46-4$ with $0.026 \mathrm{~g} \mathrm{~L}^{-1}$ day $^{-1}$. The next highest biomass productivities were observed in Chlorococcum sp. 142-5-2 and sp. 143-1 with 0.019 and $0.018 \mathrm{~g} \mathrm{~L}^{-1}$ day $^{-1}$, respectively. 
Table 3 Biomass and lipid percentages of 22 Chlorophyceae green algae

\begin{tabular}{|c|c|c|c|c|c|c|c|c|}
\cline { 2 - 9 } \multicolumn{1}{c|}{} & \multicolumn{2}{c|}{ Day 5 } & \multicolumn{2}{c|}{ Day 10 } & \multicolumn{2}{c|}{ Day 15 } & \multicolumn{2}{c|}{ Day 20 } \\
\hline Algae & Biomass & Lipid\% & Biomass & Lipid\% & Biomass & Lipid\% & Biomass & Lipid\% \\
\hline $\mathbf{5 - 1}$ & 0.17 & 19.8 & 0.17 & 43.4 & 0.10 & 69.6 & 0.30 & 39.1 \\
\hline $\mathbf{4 6 - 4}$ & 0.23 & 22.4 & 0.47 & 19.7 & 0.53 & 23.8 & 0.83 & 19.1 \\
\hline $\mathbf{6 4 - 8}$ & 0.20 & 8.5 & 0.13 & 13.6 & 0.33 & 18.9 & 0.50 & 25.3 \\
\hline $\mathbf{6 4 - 1 0}$ & 0.33 & 15.8 & 0.20 & 15.7 & 0.17 & 12.5 & 0.33 & 17.3 \\
\hline $\mathbf{6 4 - 1 2}$ & 0.63 & 28.2 & 0.23 & 15.0 & 0.20 & 16.9 & 0.57 & 17.9 \\
\hline $\mathbf{1 0 3 - 6}$ & 0.17 & 19.6 & 0.27 & 12.1 & 0.33 & 15.8 & 0.40 & 27.9 \\
\hline $\mathbf{1 0 8 - 5}$ & 0.17 & 23.4 & 0.30 & 4.3 & 0.40 & 17.3 & 0.37 & 17.6 \\
\hline $\mathbf{1 4 2 - 5 - 2}$ & 0.30 & 9.9 & 0.30 & 10.8 & 0.37 & 17.1 & 0.57 & 14.6 \\
\hline $\mathbf{1 4 3 - 1}$ & 0.17 & 23.1 & 0.33 & 17.2 & 0.33 & 24.2 & 0.60 & 14.0 \\
\hline $\mathbf{1 4 3 - 4}$ & 0.17 & 14.2 & 0.27 & 13.8 & 0.20 & 22.2 & 0.33 & 25.4 \\
\hline $\mathbf{1 4 3 - 5}$ & 0.27 & 13.1 & 0.30 & 8.1 & 0.30 & 30.7 & 0.50 & 15.1 \\
\hline $\mathbf{1 0 8 - 4}$ & 0.17 & 4.2 & 0.20 & 2.7 & 0.27 & 4.1 & 0.30 & 4.9 \\
\hline $\mathbf{1 4 3 - 6}$ & 0.30 & 10.6 & 0.33 & 4.8 & 0.20 & 5.6 & 0.30 & 10.0 \\
\hline $\mathbf{1 4 5 - 2}$ & 0.23 & 15.1 & 0.23 & 15.3 & 0.33 & 9.8 & 0.33 & 20.8 \\
\hline $\mathbf{1 4 5 - 3}$ & 0.13 & 30.4 & 0.13 & 12.9 & 0.13 & 16.2 & 0.37 & 18.0 \\
\hline $\mathbf{1 4 5 - 7}$ & 0.27 & 0.0 & 0.13 & 2.1 & 0.13 & 7.2 & 0.20 & 13.7 \\
\hline $\mathbf{1 4 8 - 1 - 1}$ & 0.37 & 1.5 & 0.37 & 0.8 & 0.10 & 8.2 & 0.37 & 5.8 \\
\hline $\mathbf{1 4 8 - 5 - 2}$ & 0.13 & 21.0 & 0.13 & 24.6 & 0.10 & 39.2 & 0.23 & 25.3 \\
\hline $\mathbf{1 5 0 - 1 - 1}$ & 0.07 & 19.0 & 0.07 & 13.3 & 0.50 & 7.3 & 0.17 & 22.8 \\
\hline $\mathbf{1 5 2 - 3}$ & 0.13 & 6.3 & 0.13 & 7.8 & 0.33 & 7.9 & 0.33 & 9.1 \\
\hline $\mathbf{1 5 5 - 1}$ & 0.10 & 31.5 & 0.13 & 30.4 & 0.33 & 19.4 & 0.37 & 21.2 \\
\hline $\mathbf{1 5 7 - 1}$ & 0.17 & 4.5 & 0.17 & 6.4 & 0.17 & 10.5 & 0.27 & 10.2 \\
\hline
\end{tabular}

Mean of three replicates per algae strain. Biomass is presented in $\mathrm{g} \mathrm{L}^{-1}$ dry weight. Lipid concentration is presented as a \% yield of dry biomass and based on Nile Red fluorescence converted by means of Triolein standard curve

In terms of lipid percentages, the highest lipid accumulation was observed between day 5 and 15 for all 22 algae strains. The mean lipid content found in all 22 Chlorophyta algae, over the 20 -day cultivation, was $16.3 \%$ of the dry biomass. On day 15 of cultivation, the highest lipid accumulation was observed in Scenedesmus sp. 143-4, Selenastrum sp. 64-10, and Chlorella sp. 5-1 with 82, 
79 , and $69 \%$ respectively. Alternatively, the biomass concentrations for these strains were relatively low, $0.2,0.17$, and $0.1 \mathrm{~g} \mathrm{~L}^{-1}$, respectively. The average lipid productivity for all 22 stains of algae was $2.22 \mathrm{mg} \mathrm{L}^{-1}$ day $^{-1}$. The highest lipid productivity was observed in Coelastrum sp. 46-4 with $5.49 \mathrm{mg} \mathrm{L}^{-1}$ day $^{-1}$. Coelastrum sp. 46-4 resulted with the overall highest biomass and lipid concentrations and was selected for screening in co-cultures with microorganisms.

3.3 Marine algae biomass and lipid productivity

Screening of nine marine algal strains isolated from South Florida for biomass and lipid productivity resulted in drastic differences among species (Figure $8,9,10)$. The mean values for the biomass and lipid concentrations for all nine strains cultivated over 20 days are $2.17 \mathrm{~g} \mathrm{~L}^{-1}$ and $2.9 \%$ of dry biomass. The highest biomass accumulation for all strains was observed on day 20 of cultivation (Figure 8). On day 20, biomass of Cricosphaera sp. 146-2-9 was the highest observed biomass followed by Vischeria sp. 146-2-8 and sp. 146-2-16 with biomass of $3.67,3.63$, and $3.33 \mathrm{~g} \mathrm{~L}^{-1}$, respectively. The algae with the highest average biomass concentrations throughout the experiment were Chlamidomonas sp.146-2-10, Vischeria sp. 146-2-16, and Cricosphaera sp. 1462-9 with 2.46, 2.37, and $2.33 \mathrm{~g} \mathrm{~L}^{-1}$ respectively (Figure 9). 


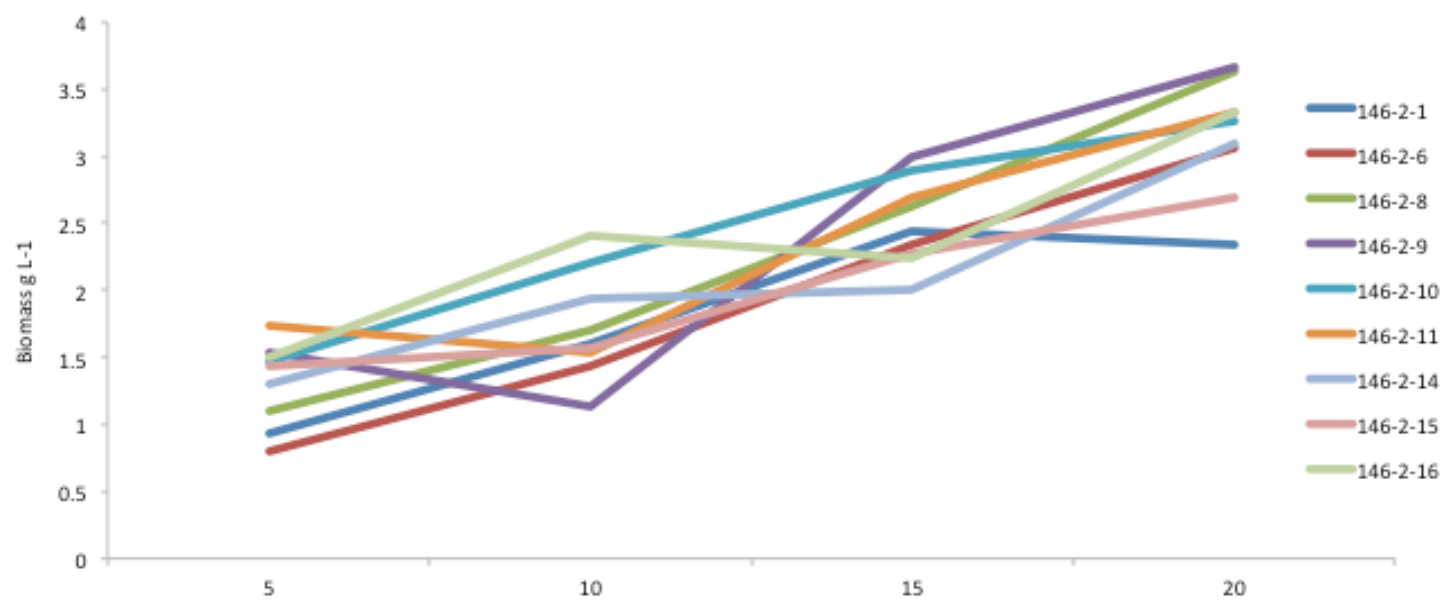

Figure 8 The biomass content $\left(\mathrm{g} \mathrm{L}^{-1}\right)$ of nine marine algae over twenty days. Data represents the mean of three replicates

In terms of lipid accumulation, the highest average lipid concentration for all nine strains was observed during day 5 and 15 of cultivation with $3.9 \%$ and $3.2 \%$, respectively. On day 5 of cultivation, Tetraselmis sp. 146-2-1 had the highest lipid concentration (8\%), followed by Chlorococcum 146-2-6 (5\%), and then Chrysotila sp. 146-2-11 (4\%). On day 15, the highest lipid concentration was observed in Vischeria sp. 146-2-16, Tetraselmis sp. 146-2-1, and Chlamidomonas sp.146-2-10 with up to $5 \%$ lipids. The algae with the highest average lipid concentrations after 20 days were Tetraselmis sp. 146-2-1 (5.0\%), Cricosphaera sp. 146-2-9 (3.5\%), and Vischeria sp. 146-2-16 (3.4\%) (Figure 9). 


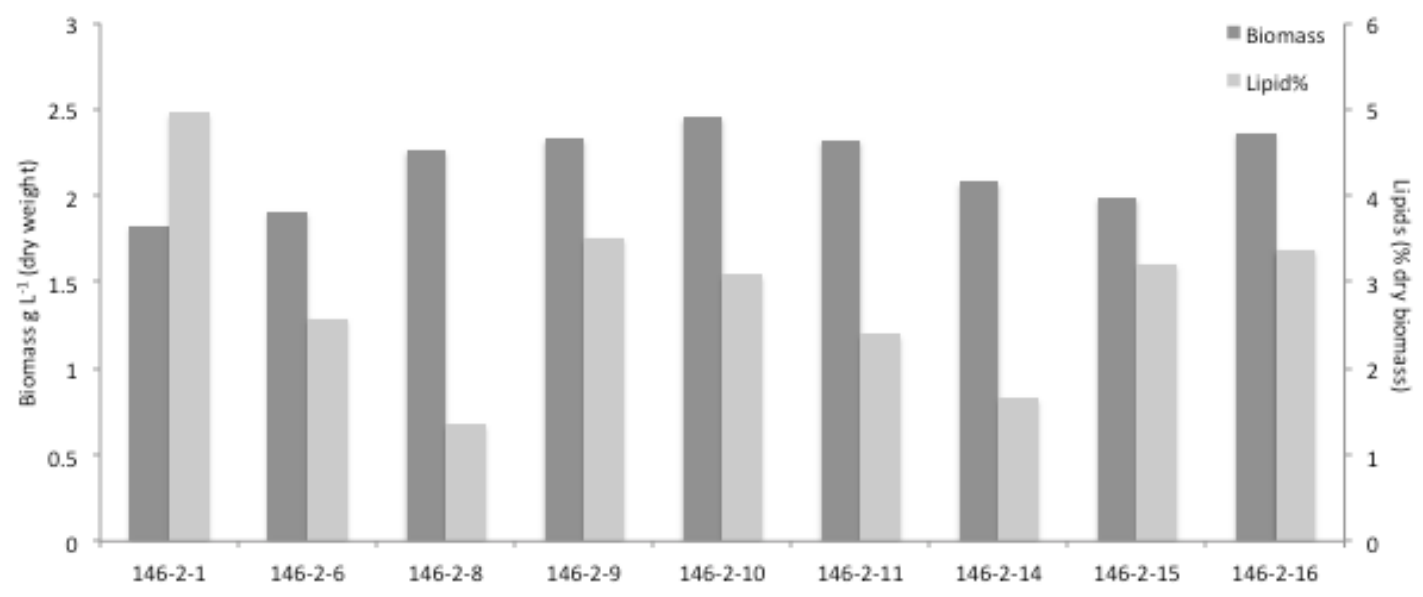

Figure 9 Average biomass ( $\mathrm{g} \mathrm{L}^{-1}$ dry weight) and lipid content (\% of dry biomass) of nine marine algae over 20 days of cultivation. Data represents the mean of three replicates over days $5,10,15$, and 20

When biomass and lipid concentration are taken into account together, however, the panel of lipid producers changes. The highest average lipid productivities for all nine strains are observed on day 5 and 10 . On day 10 , Chlorella sp. 146-2-15, Cricosphaera sp. 146-2-9, and Vischeria sp.146-2-16 had the highest lipid productivities with 15.62, 14.54, and $12.85 \mathrm{mg} \mathrm{L}^{-1} \mathrm{day}^{-1}$, respectively (Figure 10). Over the 20-day cultivation, the three algae with the highest average lipid productivities were Cricosphaera sp. 146-2-9, Chlorella sp. 146-2-15, and Tetraselmis sp. 146-2-1 with 11.15, 9.34, and $8.71 \mathrm{mg} \mathrm{L}^{-1}$ day $^{-1}$. 


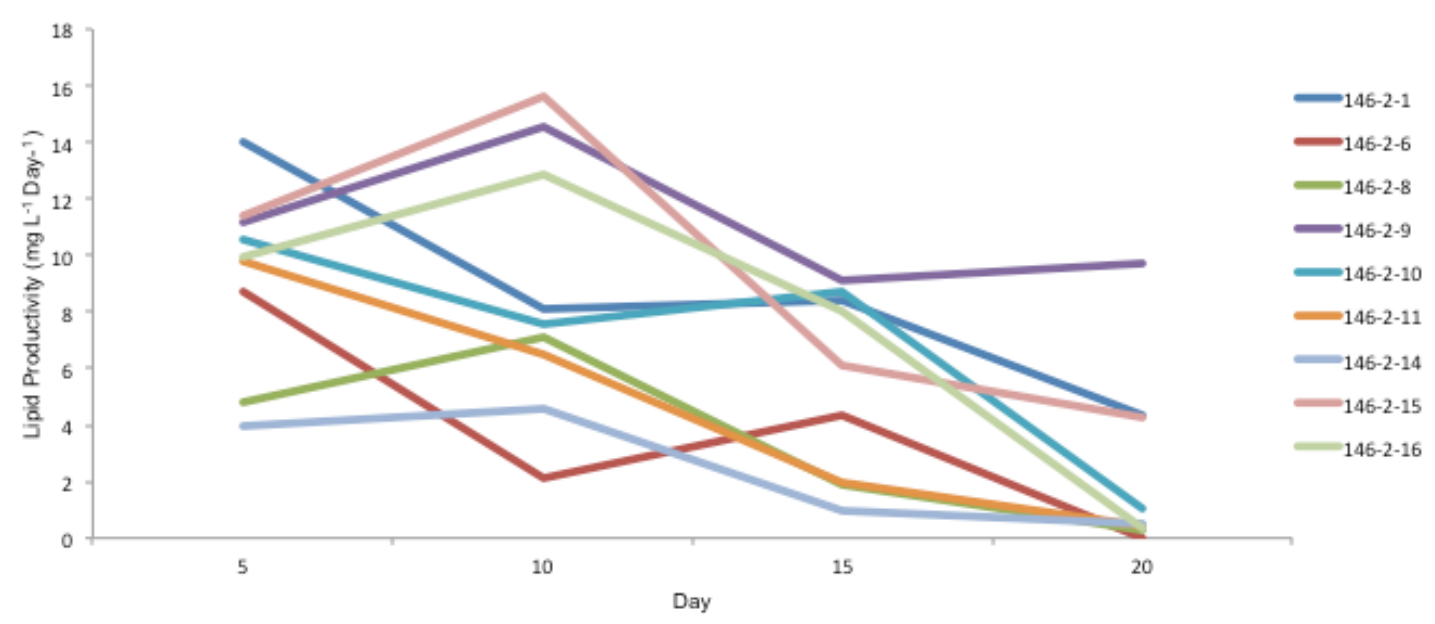

Figure 10 Lipid productivities ( $\mathrm{mg} \mathrm{L}^{-1} \mathrm{day}^{-1}$ ) of nine marine algae strains over twenty days. Data represents the mean of three replicates

Since Cricosphaera sp. 146-2-9 resulted with the highest average lipid productivity throughout the cultivation, this alga strain was chosen for further analysis in co-culture with marine fungi.

3.3 Screening co-culture experiments

3.3.1 Coelastrum sp. $46-4$ and yeast, bacteria, and $\mathrm{N}_{2}$-fixing Cyanobacteria cocultures

i. Yeast

A total of 37 strains of freshwater yeast were screened with one oleaginous algae strain, Coelastrum sp. 46-4. After one week of incubation, all yeast strains were observed to inhibit algae biomass production. In turn, during the first week, algae lipid content was increased up to $10 \%$ with a few accompanying yeast (Table 4). The second week of co-culture with yeast resulted in few strains of yeast stimulating biomass increase of Coelastrum sp. 
46-4. Alternatively, on the second week of cultivation, all yeast strains inhibited lipid accumulation in the alga. After two weeks of cultivation with yeast, the green alga responded mainly with an increase in biomass only, up to $39 \%$ with yeast strain id\# 159-1-3. As there were no positive correlation in both biomass and lipid accumulation in Coelastrum sp. 46-4 co-cultured with yeast, no further cocultivation experiments were carried out. 
Table 4 Percent difference over monocultures in the biomass and lipid content of Coelastrum sp. 46-4 with 37 yeast strains over two weeks

\begin{tabular}{|c|c|c|c|c|}
\hline \multirow{3}{*}{$\begin{array}{c}\text { Yeast } \\
\text { Strain \# }\end{array}$} & \multicolumn{4}{|c|}{$\%$ Change from monoculture of Coelastrum sp. $46-4$} \\
\hline & \multicolumn{2}{|c|}{ Week 1} & \multicolumn{2}{|c|}{ Week 2} \\
\hline & Biomass & Lipid Content & Biomass & Lipid Content \\
\hline Yo & -19.41 & 10.22 & 1.34 & -15.00 \\
\hline $145-16$ & -31.38 & 2.00 & -22.07 & -8.37 \\
\hline $148-2-1$ & -33.36 & 0.08 & -25.87 & -0.37 \\
\hline $148-2-2$ & -34.91 & -1.10 & -31.46 & -7.29 \\
\hline $148-2-3$ & -37.37 & -0.83 & -11.69 & -6.59 \\
\hline 148-2-4 & -28.00 & -2.21 & 15.01 & -13.08 \\
\hline $148-3-1$ & -37.69 & -4.13 & -12.62 & -4.51 \\
\hline 148-4-1 & -23.77 & -6.85 & 3.80 & -15.27 \\
\hline $148-4-2-1$ & -47.60 & 2.46 & -11.11 & -10.48 \\
\hline $148-4-5-1$ & -24.68 & 1.80 & -15.30 & -9.42 \\
\hline $148-4-6$ & -57.13 & 1.40 & -39.44 & -5.58 \\
\hline $148-4-6-1$ & -28.66 & -1.64 & -19.58 & -12.70 \\
\hline $149-3-5-1$ & -57.77 & 2.72 & -27.12 & -4.79 \\
\hline $151-1-3$ & -33.65 & 0.15 & 33.38 & -17.08 \\
\hline $151-3-1$ & -38.57 & 1.52 & 33.44 & -22.87 \\
\hline $151-3-2$ & -15.37 & -5.00 & 21.02 & -5.03 \\
\hline $152-3-2$ & -41.22 & 5.68 & -1.53 & -13.23 \\
\hline $152-3-3$ & -34.88 & -2.89 & -35.84 & -10.87 \\
\hline $152-3-4$ & -53.82 & -2.08 & -18.84 & -6.96 \\
\hline $152-3-5$ & -42.68 & -5.54 & -35.29 & -6.10 \\
\hline $152-3-6$ & -55.37 & -0.73 & -11.37 & -10.71 \\
\hline $152-3-7$ & -31.16 & 6.13 & -5.33 & -5.91 \\
\hline $152-3-8$ & -34.44 & -0.30 & 25.97 & -10.02 \\
\hline $152-3-9$ & -30.27 & 4.10 & -32.64 & -14.31 \\
\hline 153-1-1 & -53.54 & 3.20 & -53.94 & -6.74 \\
\hline $153-2-2$ & -48.33 & -3.79 & -21.56 & -6.67 \\
\hline 157-1-1 & -59.94 & -9.81 & -65.73 & -10.61 \\
\hline 158-2-1 & -30.21 & -5.05 & \begin{tabular}{|l|}
-11.08 \\
\end{tabular} & -15.39 \\
\hline $159-1-1$ & -60.70 & 4.82 & -58.74 & -4.92 \\
\hline $159-1-3$ & -10.01 & -8.74 & 39.44 & -11.50 \\
\hline $159-1-4$ & -34.34 & -5.26 & -13.96 & -8.72 \\
\hline $159-1-5$ & -11.30 & -6.10 & 23.09 & -2.73 \\
\hline 159-2-1 & -45.86 & -8.42 & 1.28 & -11.53 \\
\hline $159-2-1-1$ & -47.98 & -5.76 & -50.30 & -10.96 \\
\hline $159-2-2$ & -52.02 & -27.44 & -17.02 & -9.63 \\
\hline $159-2-4$ & -62.94 & -44.23 & -62.06 & -9.12 \\
\hline $159-3-2$ & -56.16 & -5.51 & -32.99 & -9.08 \\
\hline
\end{tabular}

Data represents the mean of three replicates. 


\section{ii. Bacteria}

A total of 39 strains of freshwater bacteria were screened with Coelastrum sp. 46-4. After the first week of culturing, most bacteria increased lipid content within the green alga in co-culture (Table 5). Alternatively, only four bacteria increased the biomass of Coelastrum sp. Only one bacterial isolate, sp. 2-4-2, stimulated an increase in both biomass and lipid accumulation by $0.42 \%$ and $1 \%$ respectively. During the second week of co-culturing, fourteen of the bacterial isolates stimulated up to an $8 \%$ increase in lipid content within Coelastrum sp. 46-4 in comparison with the alga grown alone. Eleven bacterial strains stimulated up to a $46 \%$ increase in the biomass concentration of the algae. In terms of stimulating both biomass and lipid content of Coelastrum sp. $46-4$ in co-culture, one bacterial strain sp. $21-9-3-2$ had a positive effect of about 26 and $10 \%$ over the algae monoculture, respectively. The bacterial strain with id\# 21-9-3-2 was chosen for further identification and analysis for co-culture experiments with Coelastrum sp. 46-4. 
Table 5 Percent difference over monocultures in the biomass content of Coelastrum sp. 46-4 with 38 bacterial strains over two weeks

\begin{tabular}{|c|c|c|c|c|}
\hline \multirow{3}{*}{$\begin{array}{l}\text { Bacteria } \\
\text { Strain \# }\end{array}$} & \multicolumn{4}{|c|}{$\%$ Change from monoculture of Coelastrum sp. $46-4$} \\
\hline & \multicolumn{2}{|c|}{ Week 1} & \multicolumn{2}{|c|}{ Week 2} \\
\hline & Biomass & Lipid Content & Biomass & $\begin{array}{c}\text { Lipid } \\
\text { Content }\end{array}$ \\
\hline 2-4-2 & 0.42 & $1.03^{*}$ & -46.94 & 5.95 \\
\hline 3-24-1 & -11.65 & -0.80 & -29.77 & 1.08 \\
\hline 3-24-2 & -34.07 & 3.13 & -39.20 & 3.92 \\
\hline 15-2-1 & -37.84 & 5.08 & -24.00 & -11.12 \\
\hline 19-2-3 & -38.09 & 3.24 & -22.52 & 0.39 \\
\hline 21-4-3-1 & -1.14 & 2.65 & -1.09 & 2.29 \\
\hline $21-9-3-2$ & -45.08 & 3.90 & $25.80^{*}$ & $9.89^{*}$ \\
\hline $21-10-20-3$ & 24.28 & -2.30 & 57.33 & -3.46 \\
\hline $30-1-8-1$ & -0.89 & 3.64 & 6.29 & -0.61 \\
\hline $33-7-1$ & -13.56 & 3.51 & -18.61 & 4.21 \\
\hline $33-10-1$ & -25.76 & 1.34 & -38.27 & 2.94 \\
\hline $46-4-1$ & -27.25 & 2.65 & -36.64 & 3.96 \\
\hline $47-2-1$ & -38.86 & 8.67 & -33.98 & 7.15 \\
\hline $47-2-1-1$ & -24.40 & -2.99 & 22.84 & -4.07 \\
\hline $47-5-1$ & -30.64 & 0.41 & 7.38 & -5.62 \\
\hline $52-1-2$ & -14.57 & 4.20 & -15.59 & -2.50 \\
\hline $108-4$ & -1.10 & 6.10 & -3.43 & -8.51 \\
\hline 108-4-1 & -36.74 & 2.69 & -41.07 & 6.88 \\
\hline 121-1-1 & -21.02 & 3.49 & -28.26 & -0.52 \\
\hline $127-2-2$ & -26.69 & 4.96 & -28.52 & 0.32 \\
\hline 127-2-3 & -21.02 & 5.66 & -34.01 & 3.03 \\
\hline $127-2-4$ & -8.31 & 7.88 & -8.79 & 8.05 \\
\hline 145-3-1 & 12.12 & -0.46 & 26.08 & -23.20 \\
\hline $145-7$ & -12.16 & 1.16 & 11.26 & -13.95 \\
\hline 146-2-1 & -21.44 & 7.63 & 8.63 & -3.95 \\
\hline $146-2-2$ & -28.56 & 7.93 & -33.65 & -3.31 \\
\hline $146-2-3$ & -31.74 & 3.96 & -40.23 & -4.86 \\
\hline $153-3-3-2$ & -34.87 & 7.35 & -45.24 & -4.33 \\
\hline 146-4 & -25.25 & 4.87 & -25.47 & -12.61 \\
\hline $148-5-2$ & -8.64 & -1.05 & 24.77 & -19.38 \\
\hline $150-1-1-1$ & -6.23 & 0.16 & -17.71 & -0.97 \\
\hline $150-1-1-2$ & 0 & -7.89 & 40.39 & -17.48 \\
\hline $150-1-1-3$ & 0.42 & -6.01 & 46.17 & -7.04 \\
\hline 152-2-2-1 & -11.65 & -4.42 & 4.14 & -14.83 \\
\hline 152-3-2-1 & -34.07 & -11.51 & -17.42 & -8.93 \\
\hline $152-3-2-2-2$ & -37.84 & 4.62 & -23.03 & -0.22 \\
\hline $152-3-2-3$ & -38.09 & -2.05 & -33.46 & -1.81 \\
\hline 158-2-2-1 & -1.14 & -10.76 & 50.88 & -21.50 \\
\hline $158-2-2$ & -45.08 & -15.41 & -38.05 & -4.39 \\
\hline
\end{tabular}

Data represents the mean of three replicates 
iii. Cyanobacteria

A total of nine $\mathrm{N}_{2}$-fixing cyanobacteria were screened with Coelastrum $\mathrm{sp}$. 46-4 to determine increases in biomass and lipid productivity in co-culture over fifteen days (Figure 11). After five days of cultivation, higher cell densities are observed in co-cultures of Coelastrum with Tolypothrix sp. 30-1-4, Calothrix sp. 38-3; 69-4, and Nostoc sp. 47-3; 113-10 over the monoculture. Higher lipid concentrations, though, are observed within co-cultures with Tolypothrix sp. 33-4, Nostoc sp. 41-1, 47-2-1, 113-10, and Calothrix sp. 113-9. On the tenth day of cultivation, higher biomass is observed in co-cultures with Calothrix sp. 38-3, 694, and Nostoc sp. 47-3, 47-2-1, 113-10. Heightened lipid concentrations are found within 7 of the 9 co-cultures including Tolypothrix sp. 30-1-4, 33-4, Calothrix sp. 38-3, 69-4, 113-9, and Nostoc sp. 47-3, 47-2-1, 69-4, 113-9. 

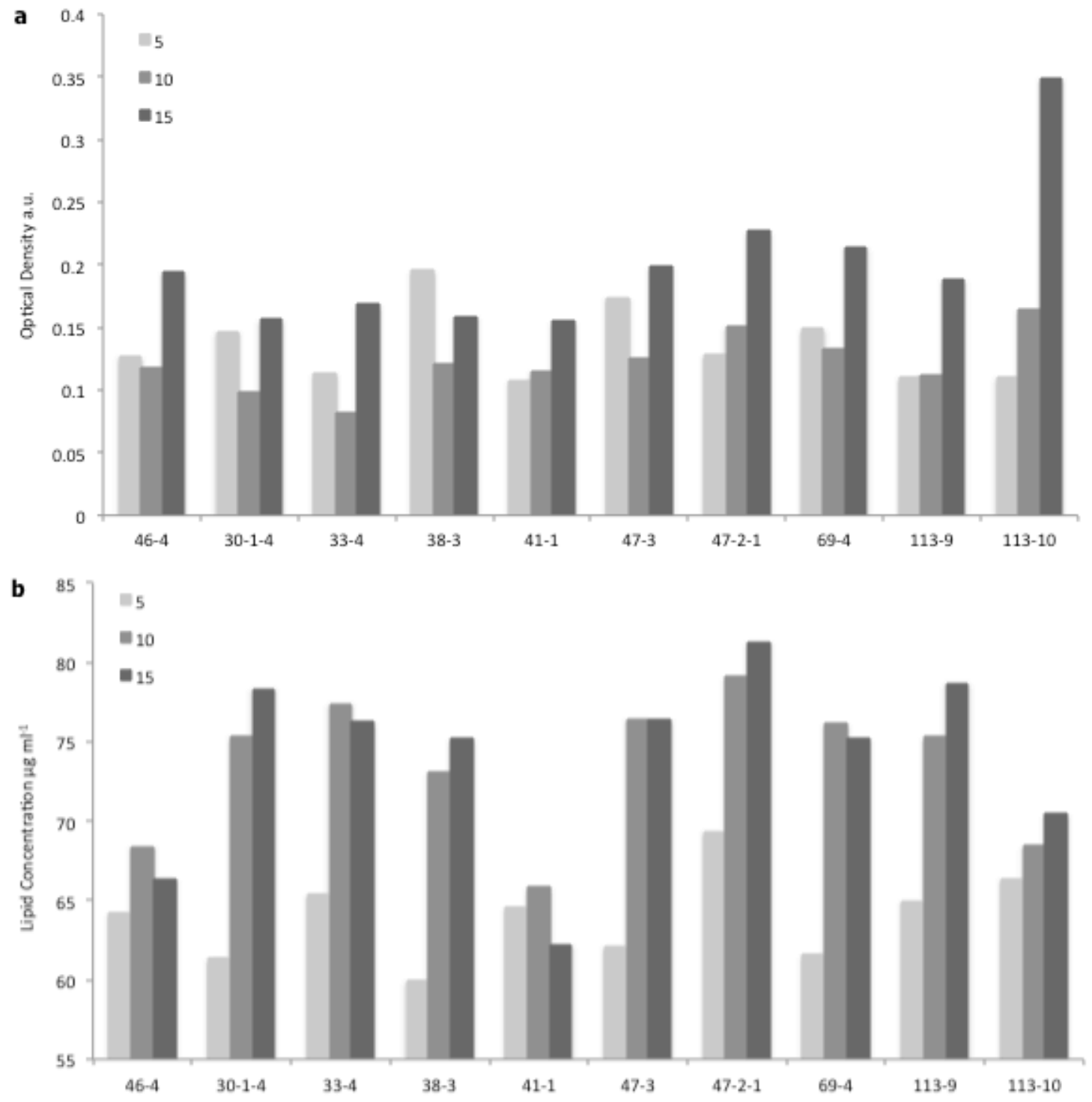

Figure 11 Optical density $(\mathrm{a} ; 600 \mathrm{~nm})$ and lipid concentration $\left(\mathrm{b} ; \mu \mathrm{g} \mathrm{ml}^{-1}\right)$ of the control Coelastrum sp. 46-4 grown in nitrogen-supplemented $\mathrm{N}+$ medium and cocultured with nine diazotrophic cyanobacteria in nitrogen deficient medium $(\mathrm{N}-)$ over 5,10 , and 15 days. Data represents the mean of three replicates

On the last day of cultivation, heightened biomass was observed in Nostoc sp. 47-3, 47-2-1, 113-10 and Calothrix sp. 69-4. Lipid concentrations in cocultures were higher than the control in eight of the nine cyanobacteria strains (Figure 11, b). When considering consistent increases in both biomass and lipid 
content in co-cultures, only Nostoc sp. 47-2-1 was able to stimulate Coelastrum as such (Figure $11 \mathrm{a}, \mathrm{b}$ ). As a result, Nostoc sp. 47-2-1 was chosen for further analysis in co-culture with Coelastrum sp. 46-4.

\subsubsection{Co-culture of Cricosphaera sp. 146-2-9 with fungi}

One marine algae strain, Cricosphaera sp. 146-2-9, was screened with 3 fungal species (146-2-F1, F3, F15) to determine increases in biomass and lipid productivities. The first week of cultivation resulted with no significant differences in the biomass and lipid content of the monocultures and co-cultures with fungi (data not shown). Results from the second week of cultivation indicated a $29 \%$ increase in the biomass concentration in Cricosphaera sp. co-cultured with fungus $146-2-\mathrm{F} 3$ with a total of $1.775 \mathrm{~g} \mathrm{~L}^{-1}$ in comparison to the control with 1.375 $\mathrm{g} \mathrm{L}^{-1}$ (Figure 12). The highest lipid accumulation, in terms of percentage of dried biomass, occurred in the control with $3.6 \%$, followed by F1 with $3.2 \%$, F15 with $2.9 \%$, and F3 with $2.7 \%$. 


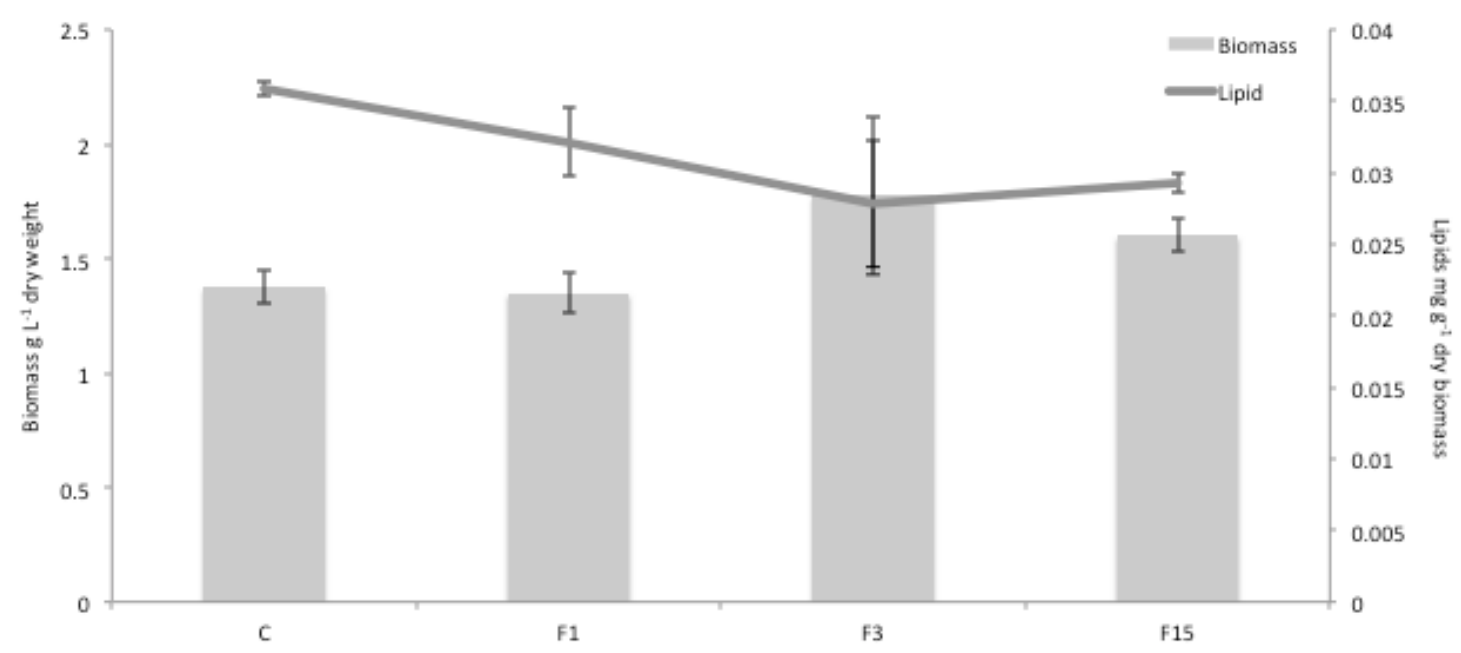

Figure 12 Biomass ( $\mathrm{g} \mathrm{L}^{-1}$ dry weight) and lipid concentration ( $\mathrm{mg} \mathrm{g}^{-1}$ dry biomass) of Cricosphaera sp. 146-2-9 (control- C) co-cultured with three fungal species $(F 1,3,15)$ on week 2 . Data represents the mean of four replicates

Despite the disparity in lipid percentages between the monoculture and co-cultures of this alga with fungus, when taking biomass and lipid content into account, namely lipid productivity, results vary. The highest lipid productivity was observed in the co-culture of the algae and F3 with $5.63 \mathrm{mg} \mathrm{L}^{-1}$ day $^{-1}$, a $13.5 \%$ increase over the control. The lowest lipid productivity was observed in the coculture with F1 with $4.14 \mathrm{mg} \mathrm{L}^{-1}$ day $^{-1}$, while that of $\mathrm{F} 15$ was $4.99 \mathrm{mg} \mathrm{L}^{-1}$ day $^{-1}$ and the control yielded $4.87 \mathrm{mg} \mathrm{L}^{-1}$ day $^{-1}$. Bright field and Nile red fluorescent microscopy of the treatments on the second week of cultivation reveals intensified Nile red fluorescence in the co-cultures of Cricosphaera sp. 146-2-9 and the fungus 146-2-F3 in comparison the control and the co-cultures with F1 and F15 fungal species (Figure 13). 


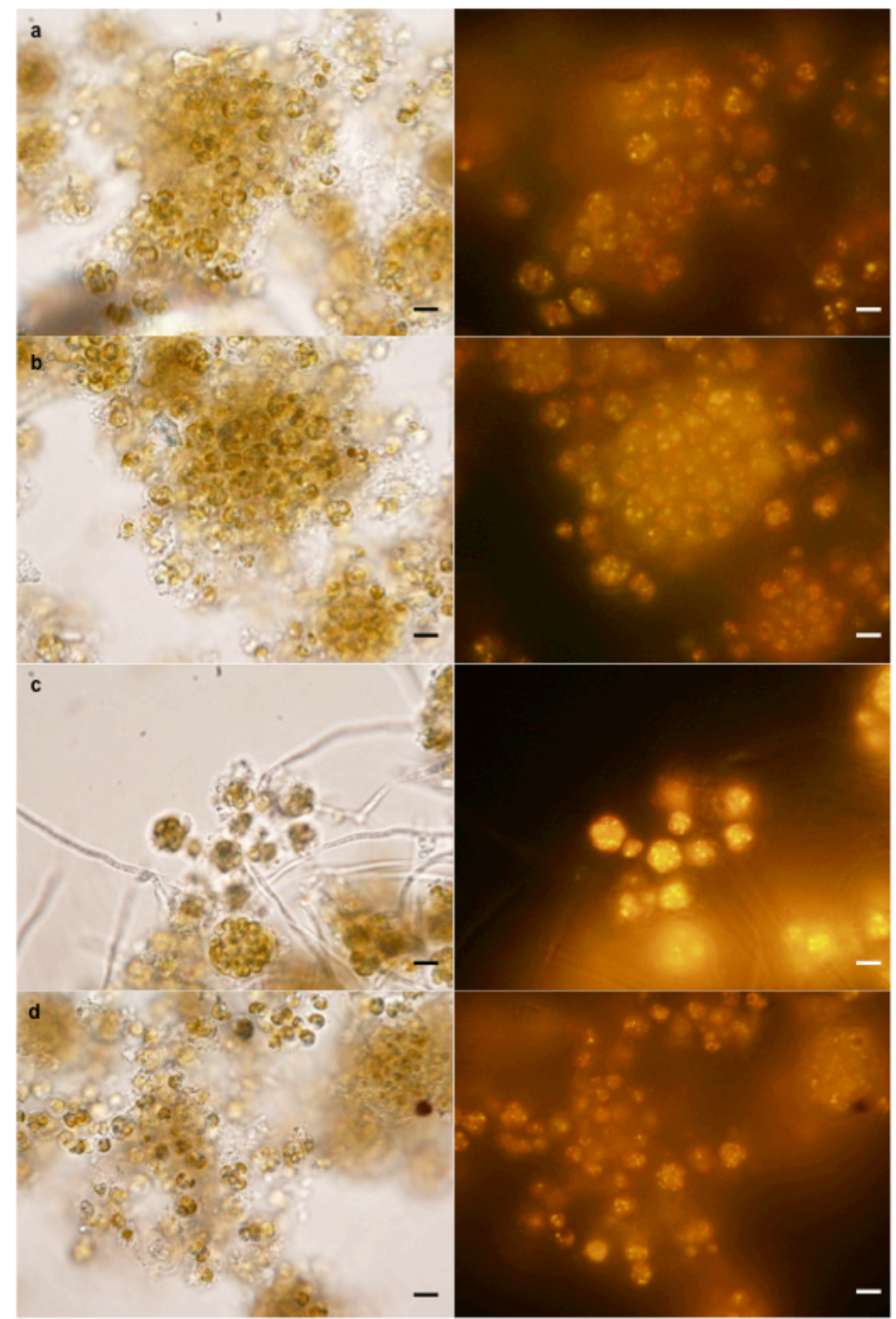

Figure 13 Bright field view (left) and Nile red fluorescence (right) of Cricosphaera sp. 146-2-9 alone in medium (a) and co-cultured with three fungal species 146-2F1, F3, F15 (b, c, d; respectively). 1000X magnification; scale bars indicate 10 $\mu \mathrm{m}$

3.4 Co-culture of Coelastrum sp. $46-4$ vs. bacterial-cell free filtrate

When the green alga Coelastrum sp. was co-cultured with Pseudomonas stutzeri and inoculated in nutrient broth and bacterial-cell free filtrate, different 
results were obtained compared to the preliminary screening (section 3.3.1). During the first week of cultivation, a significant 79 and $37.5 \%$ increase in biomass was observed in treatments with the bacterial cell-free filtrate (BS) and the nutrient broth (NB), (p-value= .002, .033; respectively; $\alpha=0.05$, Figure 14a). During the first week of cultivation, the biomass of the control was $0.2 \mathrm{~g} \mathrm{~L}^{-1}$ while that of BS and NB were 0.35 and $0.275 \mathrm{~g} \mathrm{~L}^{-1}$ respectively. There was no significant difference between the control and the algae co-cultured with the bacteria cells (M). No significant differences in lipid content was observed in all three treatments BS, M, and NB during the first week of cultivation.

The second week of co-cultivation demonstrated significant increases in the biomass content of both BS and NB treatments by 57.1 and $135.7 \%$ over the control (Figure 14b) ( $p$-value $=.035, .000$; respectively; $\alpha=0.05)$. The control accumulated $0.35 \mathrm{~g} \mathrm{~L}^{-1}$ while that of $\mathrm{BS}$ and $\mathrm{NB}$ were 0.55 and $0.825 \mathrm{~g} \mathrm{~L}^{-1}$, respectively. There was no significant difference between the control and the coculture with bacterial cells $0.325 \mathrm{~g} \mathrm{~L}^{-1}$. From figure 13, it is apparent that the lipid content, in terms of percentage of dry biomass, has no significant differences between the control, the BS, NB and M treatments. When lipid productivity, $\mathrm{mg} \mathrm{L}^{-}$ ${ }^{1}$ day $^{-1}$, is taken into account however, there is a significant increase in the BS treatment in contrast to the control, M, and NB. The average lipid productivity of BS was $4.5 \mathrm{mg} \mathrm{L}^{-1}$ day $^{-1}$, while that of the control, $\mathrm{M}$, and NB were $3.8,3.67$, and $3.67 \mathrm{mg} \mathrm{L}^{-1}$ day $^{-1}$, respectively. 

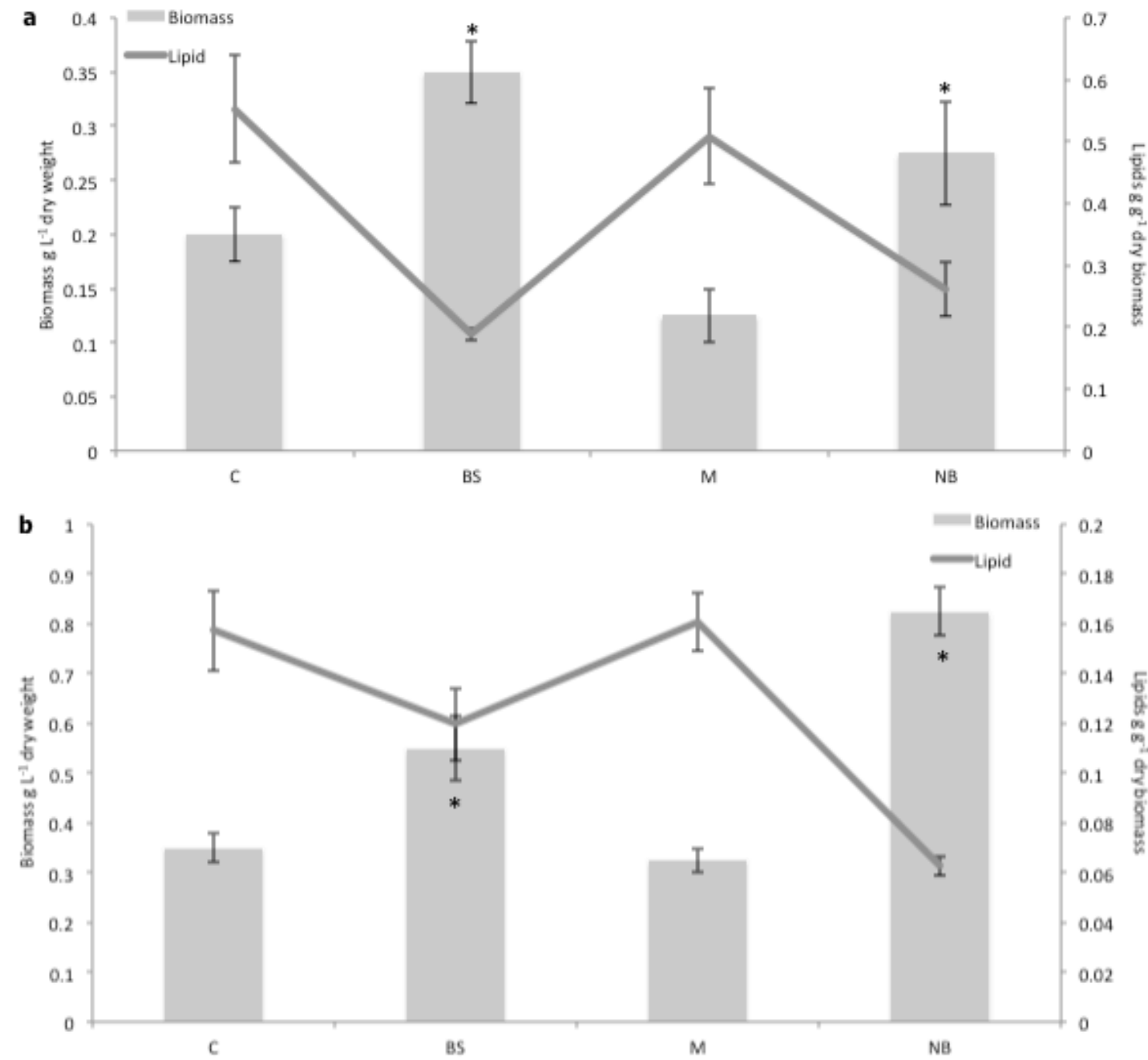

Figure 14 Biomass ( $\mathrm{g} \mathrm{L}^{-1}$ dry weight) and lipid concentration ( $\mathrm{g} \mathrm{g}^{-1}$ dry biomass) during the first (a) and second week (b) of cultivation of Coelastrum sp. 46-4 monoculture (-C) and co-culture with Pseudomonas stuzeri (- M), inoculated in bacterial cell-free filtrate (BS) and in nutrient broth (-NB). Data represents the mean of four replicates. $\left({ }^{*}\right)$ Indicates significant differences from the control $(\alpha=0.05)$

Bright field and Nile red fluorescent microscopy of the treatments on the second week of cultivation reveals a heightened lipid fluorescence from most of the cells cultivated in the bacteria-cell free filtrate and some from the $M$ 
treatment, while the C and NB treatments showed little lipid fluorescence and mostly red chlorophyll autofluorescence (Figure 15). 


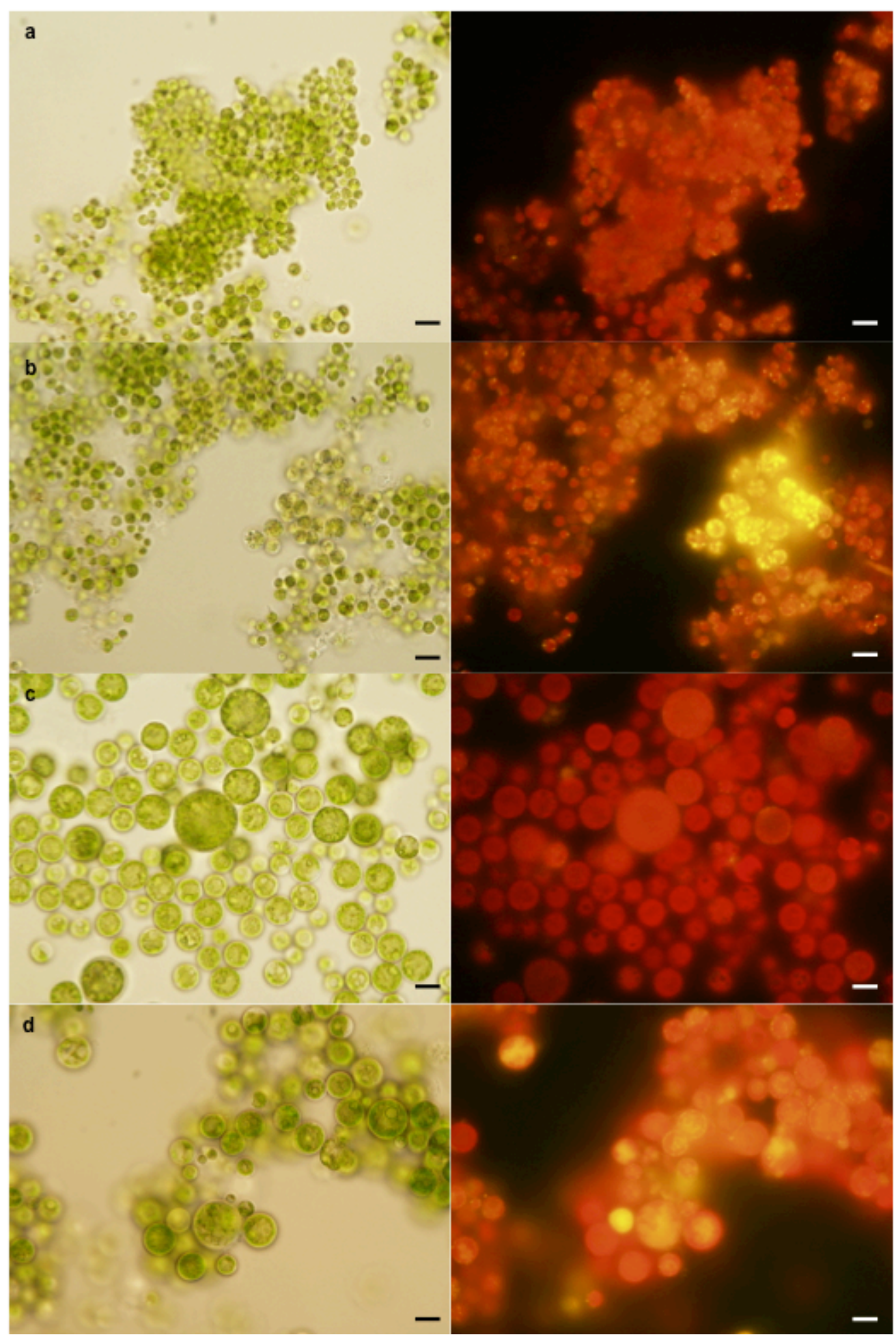

Figure 15 Bright field view (left) and Nile red fluorescence (right) of Coelastrum sp. 46-4 alone in medium (a), co-cultured with Pseudomonas stutzeri (b), in nutrient broth (c), and inoculated in bacteria cell-free filtrate (d). 1000X magnification; scale bars indicate $10 \mu \mathrm{m}$ 
3.5 Co-culture of Coelastrum sp. 46-4 and Nostoc sp. 47-2-1

During the first week of cultivation, the highest biomass was observed in the co-cultures of Coelastrum sp. and Nostoc sp. 47-2-1 with $0.250 \mathrm{~g} \mathrm{~L}^{-1}$ while the control had a yield of $0.125 \mathrm{~g} \mathrm{~L}^{-1}$. The mean biomass of the co-culture was significantly higher than the monoculture with a $100 \%$ difference $(p$-value $=.017$; $a=0.05$ ). The lipid content for both treatments resulted with $47 \%$ for the control in $\mathrm{N}+$ medium and $18 \%$ for the co-culture with diazotrophic cyanobacteria in $\mathrm{N}$ medium (Figure 16a). The second week of cultivation had similar results, with the highest biomass observed in the co-culture with $0.325 \mathrm{~g} \mathrm{~L}^{-1}$ while the control yielded $0.3 \mathrm{~g} \mathrm{~L}^{-1}$ (Figure 16b). Lipid yields resulted with $27 \%$ within the control and $20 \%$ for the co-culture. However, there were no significant differences between the mean biomass and lipid content, or productivities of the monocultures and co-cultures. Taking lipid productivity into account, the control in $\mathrm{N}+$ consistently had higher lipid productivities than the co-culture with 8.4 and $5.9 \mathrm{mg} \mathrm{L}^{-1}$ day $^{-1}$ over the two-week cultivation whereas the co-culture resulted with 6.6 and $4.6 \mathrm{mg} \mathrm{L}^{-1}$ day $^{-1}$. 

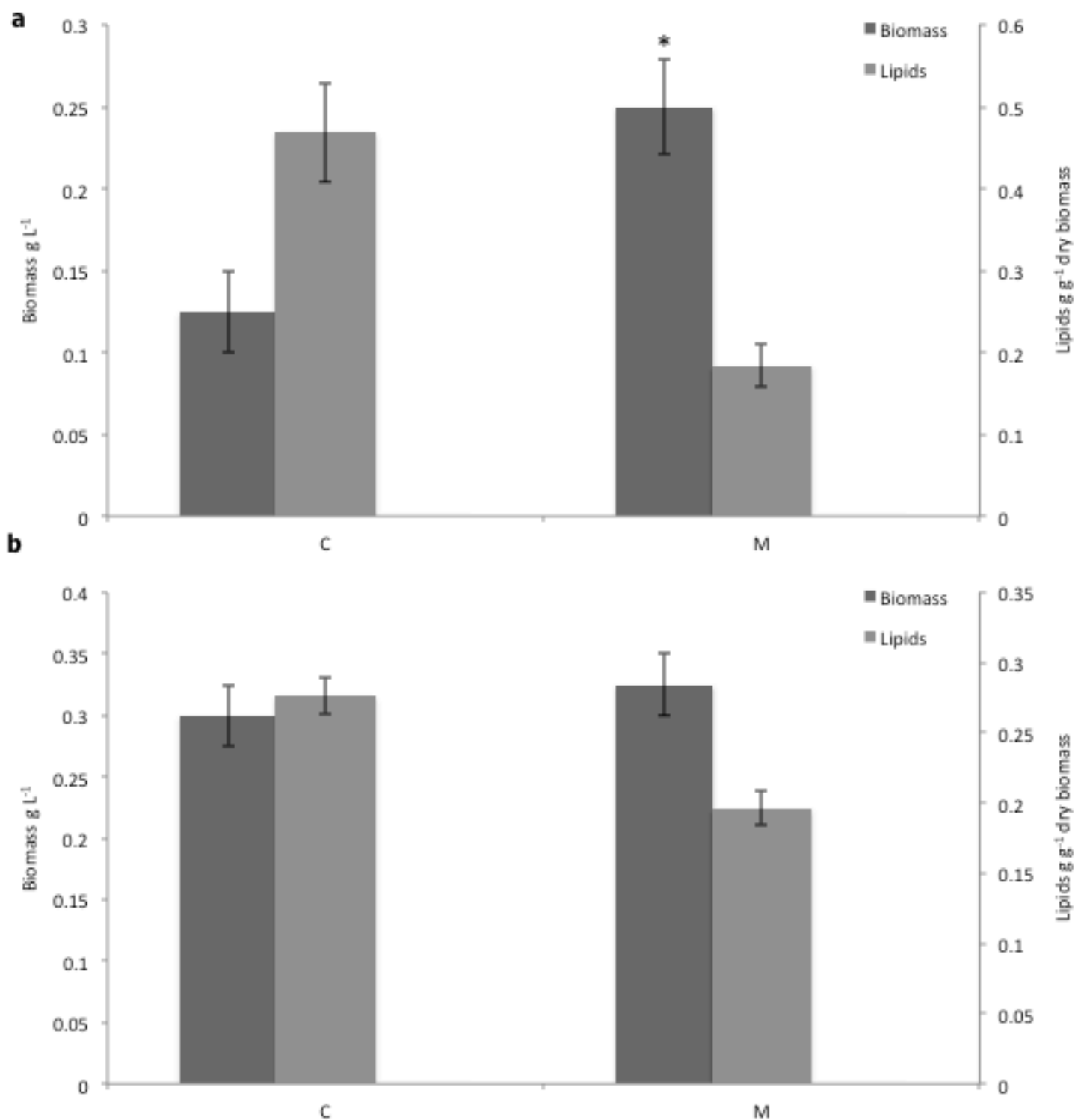

Figure 16 Biomass ( $\mathrm{g} \mathrm{L}^{-1}$ dry weight) and lipid concentration ( $\mathrm{mg} \mathrm{g}^{-1}$ dry biomass) during the first (a) and second week (b) of cultivation of Coelastrum sp. 46-4 in monocultures (-C) and co-cultured with Nostoc sp. 47-2-1 (-M). Data represents the mean of four replicates. $\left({ }^{*}\right)$ Indicates significant differences $(\alpha=0.05)$

Bright field and Nile red fluorescent microscopy of the co-cultures of Coelastrum sp. 46-4 and diazotrophic Nostoc sp. 47-2-1 on the second week of 
cultivation reveals no significant difference in the lipid fluorescence from the cells cultivated in the either monoculture or co-culture (Figure 17).

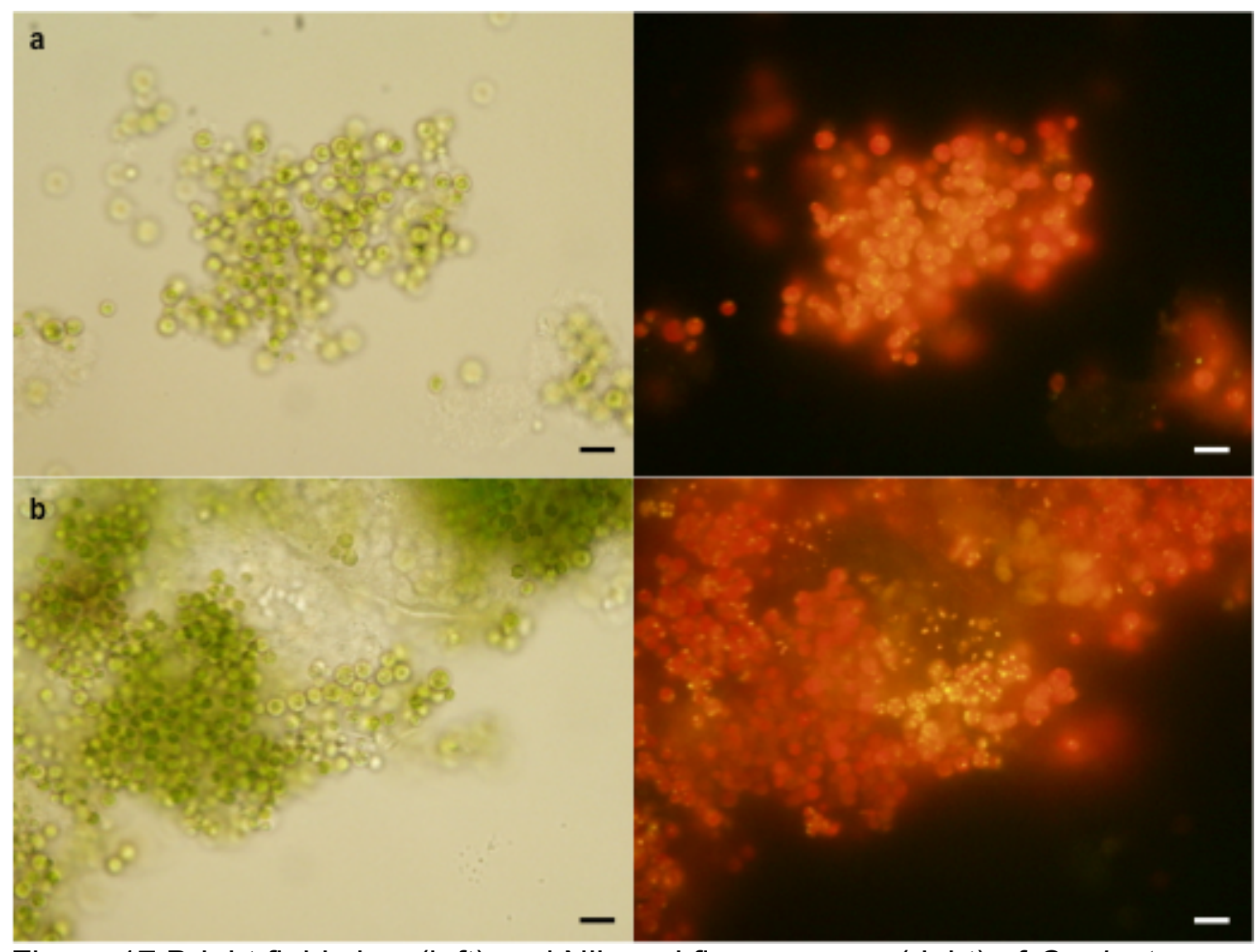

Figure 17 Bright field view (left) and Nile red fluorescence (right) of Coelastrum sp. 46-4 alone in medium (a), co-cultured with Nostoc sp. 47-2-1 (b). 1000X magnification; scale bars indicate $10 \mu \mathrm{m}$ 
3.6 Co-culture of Cricosphaera sp. 146-2-9 vs. fungal cell-free filtrate

The first week of cultivation indicated no significant difference between the biomass concentrations of the co-culture of Cricosphaera sp. with Fusarium sp., GPY, and FS treatments versus the monoculture C (Figure 18a). The co-culture had a biomass yield of $1.626 \mathrm{~g} \mathrm{~L}^{-1}$ while $\mathrm{C}$ had $1.4 \mathrm{~g} \mathrm{~L}^{-1}$, and the cultures treatments FS had $1.125 \mathrm{~g}$ and GPY with $1.05 \mathrm{~g} \mathrm{~L}^{1}{ }^{1}$. The highest lipid content was observed in the FS treatment with almost $6 \%$ and the remaining treatments below $5 \%$. The lipid content within the FS treatment was significantly higher than the control with a $70.99 \%$ difference ( $p$-value $=.004 ; \alpha=0.05)$.

During the second week of cultivation, however, there was no significant difference between the biomass content $C$ and the treatments $M$ and GPY (Figure 18b). The highest biomass was observed in the GPY treatment with $1.675 \mathrm{~g} \mathrm{~L}^{-1}$. The co-cultures had a biomass yield of $1.4 \mathrm{~g} \mathrm{~L}^{-1}$, the control with 1.3 $\mathrm{g} \mathrm{L}^{-1}$, and the FS treatment with $0.875 \mathrm{~g} \mathrm{~L}^{-1}$. In terms of lipid content, the highest mean lipid accumulation occurred within the FS treatment with $8.5 \%$, while the remaining treatments did not result with lipid contents above $3.6 \%$. The lipid content within the FS treatment was significantly higher than the monoculture by $120.67 \%(p-$ value $=.000 ; \alpha=0.05)$.

In terms of lipid productivity, the FS treatment consistently achieved the highest productivities consistently throughout the experiment. The FS treatment had a productivity of $9.2 \mathrm{mg} \mathrm{L}^{-1}$ day $^{-1}$ on the first, and $4.8 \mathrm{mg} \mathrm{L}^{-1}$ day $^{-1}$ and 
second week of cultivation, whereas the remaining treatments did not exceed 6.7 $\mathrm{mg} \mathrm{L}^{-1}$ day $^{-1}$.
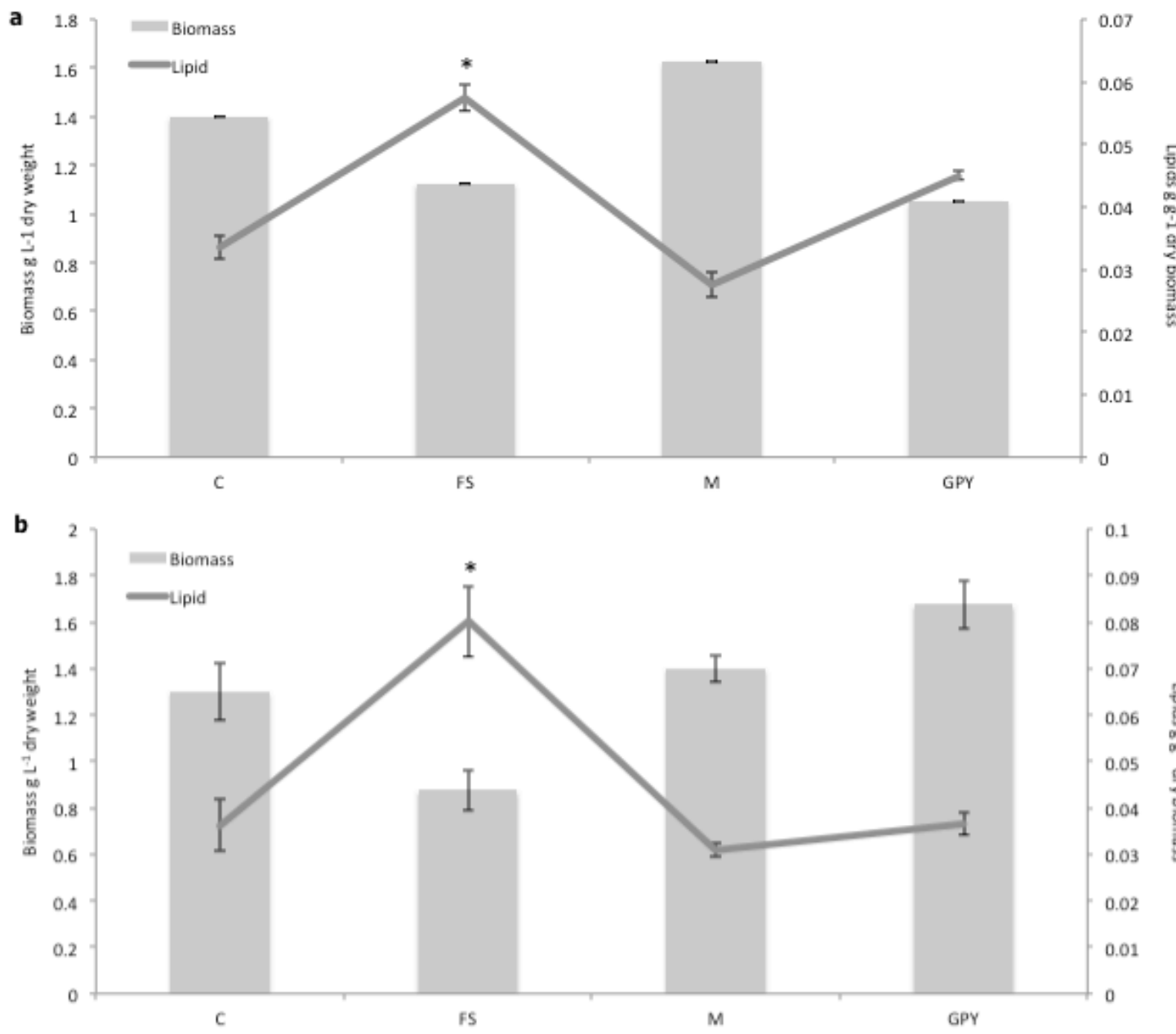

Figure 18 Biomass ( $\mathrm{g} \mathrm{L}^{-1}$ dry weight) and lipid concentration ( $\mathrm{g} \mathrm{g}^{-1}$ dry biomass) during the first (a) and second week (b) of cultivation of Cricosphaera sp. 146-2-9 monoculture (-C) and co-culture with Fusarium sp.146-2-F3 (- M), inoculated in fungal cell-free filtrate (FS) and in marine GPY (-GPY). Data represents the mean of four replicates. $\left(^{*}\right)$ Indicates significant differences from the control $(\alpha=0.05)$

Bright field and Nile red fluorescent microscopy of the treatments on the second week of cultivation shows a heightened lipid fluorescence from most of the cells cultivated in the fungal-cell free filtrate, whereas the control showed little 
lipid fluorescence (Figure 18). The algae cells co-cultured with the fungal cells revealed a higher lipid concentrations in comparison to the control. The lipid bodies within individual algal cells in the FS treatment showed very large singular lipid bodies in contrast to the smaller multiple lipid bodies accumulated within the cells of the GPY treatment. 


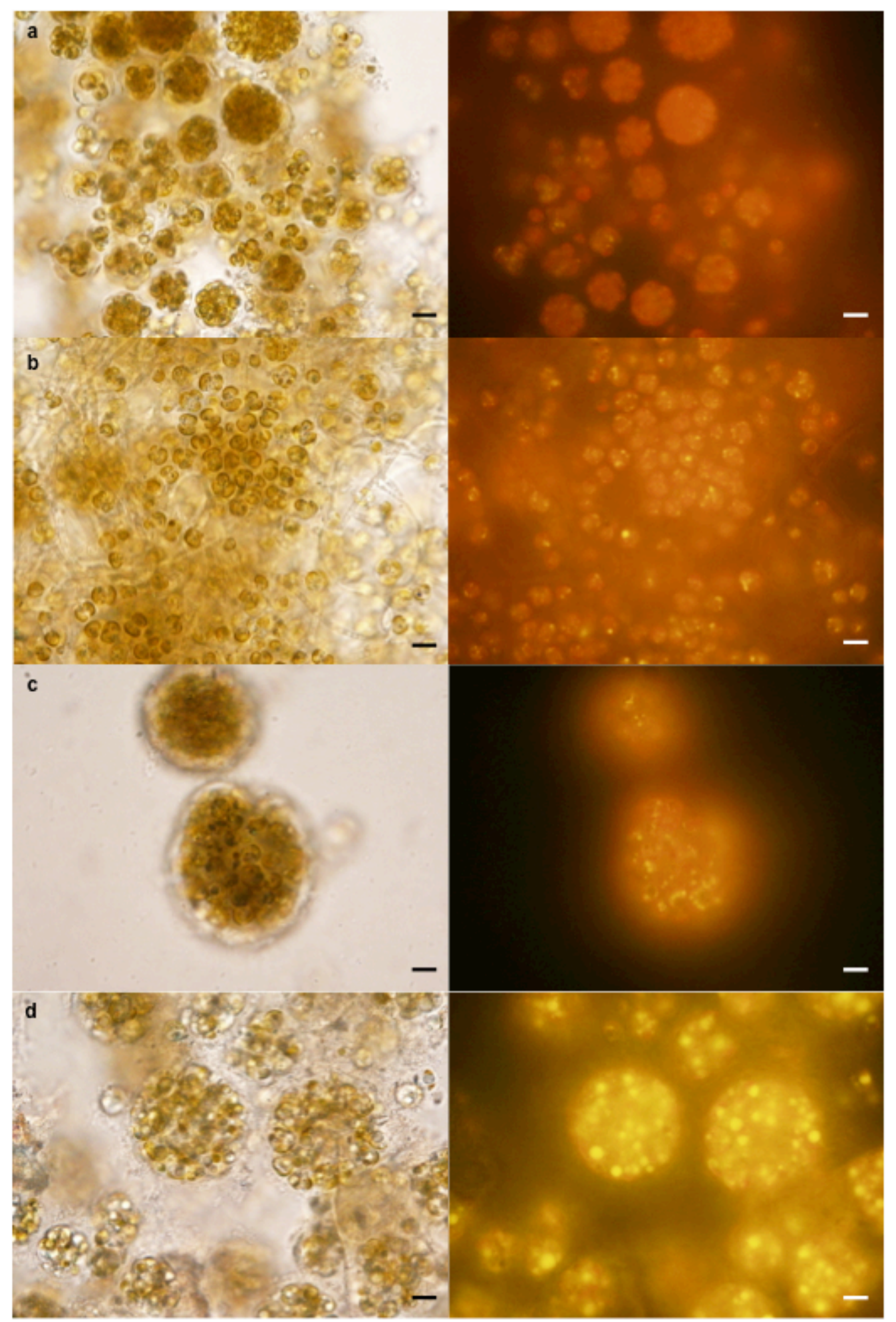

Figure 19 Bright field view (left) and Nile red fluorescence (right) of Cricosphaera sp. 146-2-9 alone in medium (a), co-cultured with Fusarium sp. 146-2-F3 (b), in GPY (c), and inoculated in fungal cell-free filtrate (d). 1000X magnification; scale bars indicate $10 \mu \mathrm{m}$ 
3.7 Gravimetric lipid analysis of Coelastrum sp. 46-4 and Cricosphaera sp. 1462-9 in mono- and co-cultures and microorganism-cell free filtrate

\subsubsection{Co-cultures of Coelastrum sp. 46-4 with Pseudomonas stutzeri}

The biomass of monocultures of Coelastrum, co-cultures with Pseudomonas stutzeri, inoculated in NB and bacterial-cell free filtrate was harvested on the second week of cultivation and the total neutral lipid content was extracted, quantified and then transesterified; results are shown in Figure 20. The highest biomass yield occurred in the NB treatment and co-culture with bacterial cells having biomass yields of 0.360 and $0.266 \mathrm{gL}^{-1}$, respectively. The control resulted with a biomass of $0.187 \mathrm{gL}^{-1}$ while that of the BS treatment yielded $0.155 \mathrm{gL}^{-1}$. Gravimetric lipid analysis shows that the highest lipid content was found in the BS treatment with $35.01 \%$, while the control has a yield of $18.96 \%$, followed by NB with $21.52 \%$, and the co-culture with $17.08 \%$. Of the lipid extracted from the biomass, after transesterification, the $C$ had a FAME yield of $16.21 \%$, the BS treatment with $29.73 \%$, M with $16.57 \%$, and lastly NB with $10.59 \%$. 


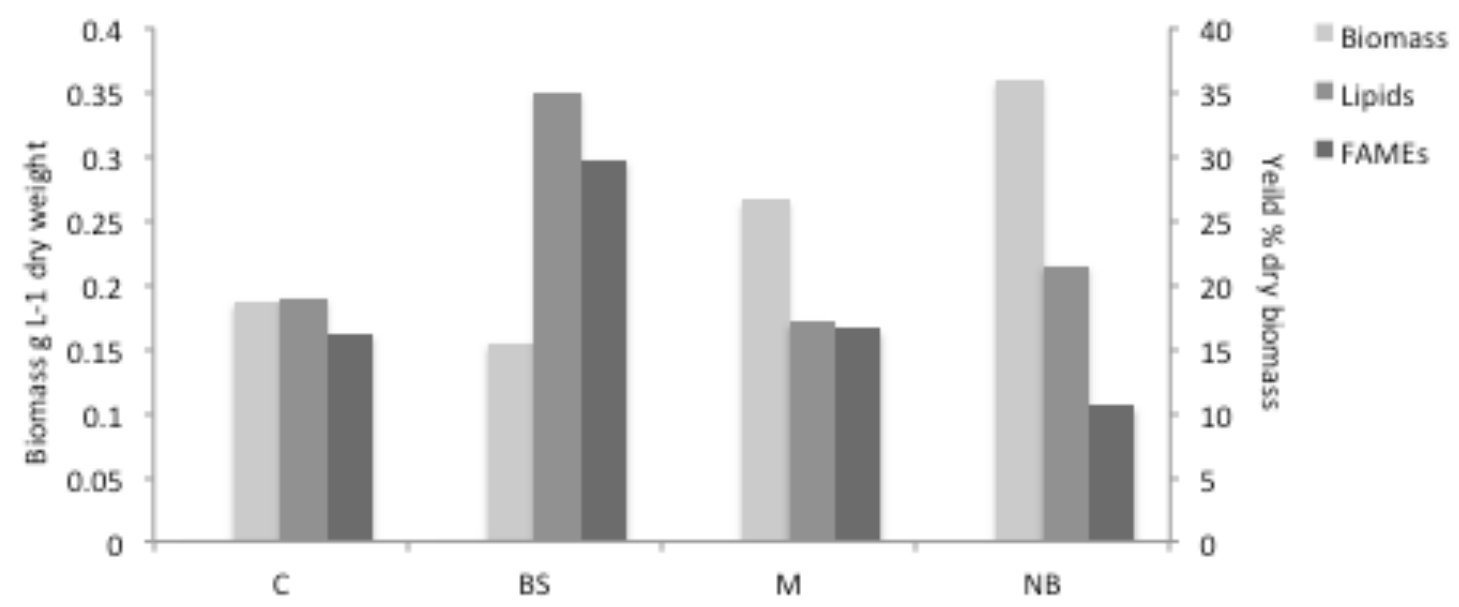

Figure 20 The biomass ( $\mathrm{g} \mathrm{L}^{-1}$ dry weight), total lipid and FAME content (\% yield of dry biomass) of Coelastrum sp. 46-4 in monoculture (-C), co-cultured with Pseudomonas stutzeri (-M), and inoculated in bacterial cell-free filtrate (-BS) and nutrient broth (-NB)

\subsubsection{Co-cultures of Coelastrum with Nostoc sp. 47-2-1}

At the end of a two-week cultivation, the biomass of Coelastrum monocultures and co-cultures with Nostoc sp. 47-2-1 was harvested, dried, and extracted for total neutral lipid content. The biomass content of Coelastrum sp. 46-4 grown in $\mathrm{N}+$ resulted with $0.409 \mathrm{~g} \mathrm{~L}^{-1}$ while that of the co-culture was 0.122 $\mathrm{g} \mathrm{L}^{-1}$. Gravimetric lipid analysis indicates that the highest lipid content was found in the co-culture with $22.24 \%$ while the monoculture had a $15.24 \%$ lipid content. 


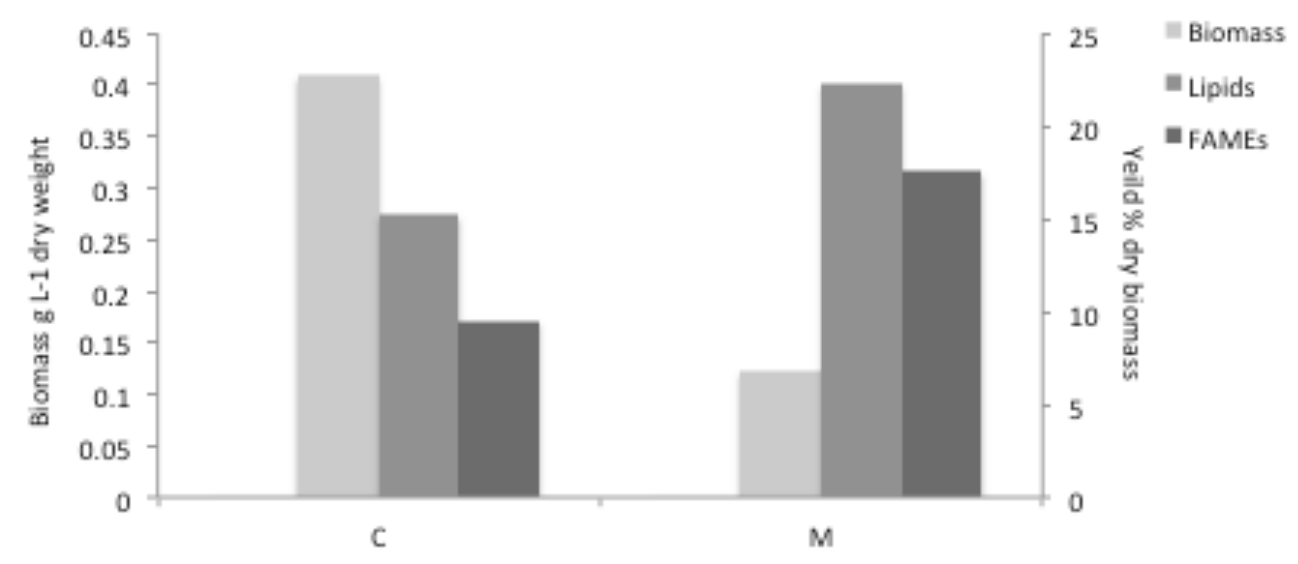

Figure 21 The biomass ( $\mathrm{g} \mathrm{L}^{-1}$ dry weight), total lipid and FAME content (\% yield of dry biomass) of Coelastrum sp. 46-4 in monoculture (-C) and co-cultured with Nostoc sp. 47-2-1 (-M)

Gravimetric FAME analysis of Coelastrum sp. 46-4 in monoculture and coculture with Nostoc sp. $47-2-1$ indicates 9.54 and $17.58 \%$ of the total lipids within the biomass are methyl esters, respectively.

3.7.3 Co-culture of Cricosphaera sp. 146-2-9 with Fusarium sp. 146-2-F3

After two weeks of cultivation, the biomass of Cricosphaera sp. monocultures and co-cultures with Fusarium sp. 146-2-F3, inoculated in GPY and fungal-cell free filtrate was harvested and the total neutral lipid was quantified and subsequently transesterified. The highest biomass concentration was observed in the co-cultures of Cricosphaera sp. and the fungus with a yield of $0.541 \mathrm{~g} \mathrm{~L}^{-1}$. The monoculture of Cricosphaera sp. had a biomass yield of 0.539 $\mathrm{g} \mathrm{L}^{-1}$, while the treatment inoculated in fungal-cell free filtrate and GPY had a biomass of $0.211 \mathrm{gL}^{-1}$ and $0.130 \mathrm{~g} \mathrm{~L}^{-1}$, respectively. The highest lipid content 
occurred in the FS and GPY treatments with 35.01 and $21.25 \%$ respectively. The co-cultures treatment $\mathrm{M}$ yielded $16.08 \%$ while the monoculture $\mathrm{C}$ yielded $11.2 \%$.

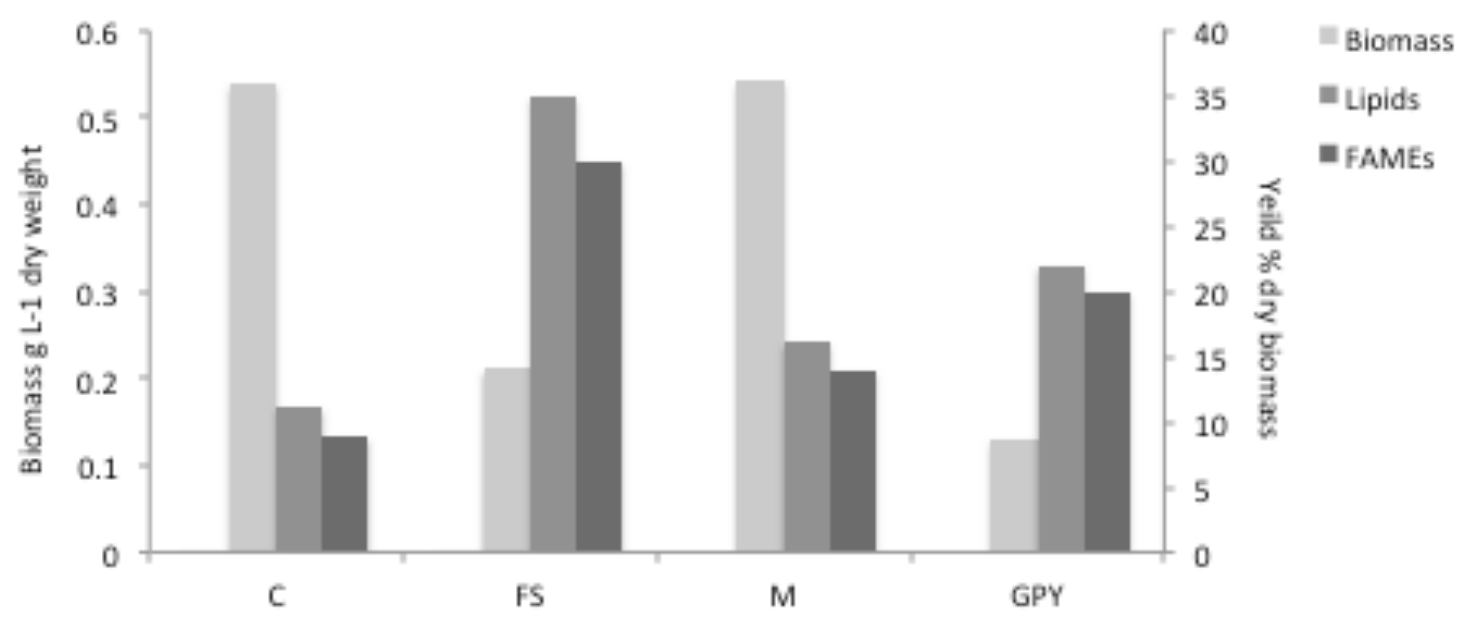

Figure 22 The biomass ( $\mathrm{g} \mathrm{L}^{-1}$ dry weight), total lipid and FAME content (\% yield of dry biomass) of Cricosphaera sp. 146-2-9 in monoculture (-C), co-cultured with Fusarium sp. 146-2-F3 (-M), and inoculated in fungal cell-free filtrate (-FS) and marine GPY (-GPY)

Gravimetric FAME analysis indicates that $8.89 \%$ of Cricosphaera sp. total lipids within the biomass has the capability of undergoing transesterification. The highest ester yield was found in the FS and GPY treatments with $30.23 \%$ and 20.67\% FAME content respectively. The co-cultures with Fusarium sp. 146-3-F3 had an ester yield of $13.99 \%$ after transesterification. 


\section{CHAPTER 4}

\section{DISCUSSION}

\subsection{Using South Florida algae for biofuels}

Choosing algal species with characteristics including simultaneous high biomass and lipid content is fundamental to using algae as a feedstock for biofuel. (Griffiths and Harrison 2009). Besides superior lipid productivity, the algal species applied should be native and adapted to the regional temporal variations to succeed in cultivation. When screening Florida freshwater Chlorophyta microalgae, the average lipid content of the 20 different strains (excluding reference strains) throughout the twenty-day cultivation was found to be $16.3 \%$ of the dry biomass. The average lipid content within the algae isolated from South Florida in comparison to the data collected by Griffiths and Harrison (2009) on the lipid content of about 25 species of algae from multiple literature resources, the average lipid content for Chlorophyta calculated was $23 \%$, a figure not far from results presented here. In terms of lipid productivity, the average lipid productivity observed from the 22 different algal strains was $3.73 \mathrm{mg} \mathrm{L}^{-1}$ day $^{-1}$ $(n=21)$. This average lipid productivity is very low compared to the reported

average of $50 \mathrm{mg} \mathrm{L}^{-1}$ day $^{-1}$ for 55 species of algae but comparable to those found by others with values between 2.6-38.7 $\mathrm{mg} \mathrm{L}^{-1} \mathrm{day}^{-1}$ (Hempel et al. 2012). The average lipid productivity is considerably low as a result of low biomass production. The average biomass productivity for all freshwater algae isolated 
and used in this research was $0.025 \mathrm{~g} \mathrm{~L}^{-1}$ day $^{-1}$, which was low compared to the average reported biomass values of $0.05-0.5 \mathrm{~g} \mathrm{~L}^{-1}$ day $^{-1}$ for some 35 strains (Hempel et al. 2012) and 0.03-0.59 $\mathrm{g} \mathrm{L}^{-1}$ day $^{-1}$ for others (Griffiths and Harrison 2009). The average biomass content reported in this work is low for algae in closed cultivation possibly because of slowed growth in axenic culture or reduced growth after the application of antibiotics (Cho et al. 2015; Watanabe 2005).

Screening of South Florida saltwater algae revealed that the average biomass and lipid productivities were $0.190 \mathrm{~g} \mathrm{~L}^{-1}$ day $^{-1}$ and $6.49 \mathrm{mg} \mathrm{L}^{-1}$ day $^{-1}$, respectively. The moderate growth rates observed in these marine species in comparison to the freshwater species might be a result of a slightly lower doubling time in marine species. The marine algae overall lipid content for all nine strains used in this research is $2.9 \%$, a figure drastically lower that what is reported for marine or saltwater algal species; Griffiths and Harrison report an overall $24 \%$ lipid content for marine species under nutrient-replete conditions. This reduced lipid production within these species could reflect a natural lipid productivity within the isolated species, or issues with growth in axenic culture, or high concentration of salts within the medium. The marine algae species used in this research were isolated from the coastline of Florida near estuaries and bays (Figure 3), where salinity fluctuates as a function of distance from the coast and temporal oscillations, and drives different populations of algae (Nodine and Gaiser 2004). These changing salinity states could provide varying optimal conditions for the algae, whereas laboratory conditions combined with BG11 
Marine with constant high salinity (3.5\%) might have negative effects on cell lipid production.

From the 22 freshwater and nine marine algae strains used in this research, freshwater Coelastrum sp. 46-4 and saltwater Cricosphaera sp. 146-29 were both selected on account of higher productivities in biomass and lipids. Coelastrum sp. $46-4$ resulted with biomass and lipid productivities of $0.026 \mathrm{~g} \mathrm{~L}^{-1}$ day $^{-1}$ and $5.49 \mathrm{mg} \mathrm{L}^{-1}$ day $^{-1}$. Cricosphaera sp. 146-2-9 resulted with an average to good biomass productivity of $0.20 \mathrm{~g} \mathrm{~L}^{-1}$ day $^{-1}$ with lipid productivity and $11.15 \mathrm{mg}$ $\mathrm{L}^{-1}$. These two algal species were selected for co-culture experiments with varying microorganisms including yeast, bacteria, diazotrophic cyanobacteria, and fungi to stimulate both biomass and lipid production simultaneously.

\subsection{Screening of co-cultures of algae and microorganisms}

When screening freshwater Coelastrum sp. 46-4 with 37 yeast, 39 bacteria, and nine diazotrophic cyanobacteria, positive correlations was observed between bacteria and cyanobacteria only. The yeast co-cultured with Coelastrum sp. 46-4 inhibited or stimulated either biomass or lipid content, but never the two concurrently (Table 4). Increases in algae biomass in combination with yeast cells can be explained in part by generation of yeast $\mathrm{CO}_{2}$, since the interchange of gases is known to stimulate mixed cultures (Wang et al. 2016). With the proliferation of the algae cells, though, widespread reduced lipid contents were observed, as algae do not generally store lipids under desirable conditions. Alternatively, areas where yeast repressed algal biomass, small increases in lipid 
content were observed. The inhibition of algae growth could have been a result of yeast overgrowth and resultant $\mathrm{CO}_{2}$ concentration, since it is understood that high concentration of the aqueous gas impedes algae photosynthesis (Lee and Tay 1991; Cai et al. 2007). Yeast extracellular compounds could have also accounted for either stimulation or inhibition of algae cell and lipid growth. Several organic acids produced by yeasts, including pyruvic and acetic acids, can be recycled by algae with resultant cell and lipid proliferation (Xue et al. 2010). Organic compounds produced by yeast in combination with yeast overgrowth may alternatively have impeded algae growth, and could provide an explanation for the observed widespread growth and lipid inhibition with Coelastrum sp. 46-4 (Table 4).

Screening the alga Coelastrum sp. 46-4 with 39 strains of bacteria revealed only one bacterial strain, \# 29-1-3-2 capable of stimulating both biomass and lipid content within the algae (Figure 5). First and second week of co-culturing of bacteria and this alga species indicated varied effects among bacterial strains where a greater portion of the bacteria tested reduced the growth of Coelastrum sp. 46-4 in co-culture, results that are consistent with screening for growth-promoting bacteria with algae (Le Chevanton et al. 2013). The demonstrated reduced growth in the algae may be caused by the lack of production of bacterial growth-promoting compounds or inhibition by cellular exudates (Fukami et al 1997; Park et al. 2008). Bacteria sp. 29-1-3-2 was capable of stimulating both the biomass and lipid content within the algal cell by $25.8 \%$ and $9.9 \%$, respectively (Table 5). Biolog identification identified the 
bacterium as Pseudomonas stutzeri, a gram-negative bacterium. This bacterium was selected for further analysis of its extracellular compounds on the growth and lipid accumulation within Coelastrum sp. 46-4.

Pairing of Coelastrum sp. 46-4 with nine strains of $\mathrm{N}_{2}$-fixing cyanobacteria in nitrogen-deficient medium indicated that the biomass and lipid content of green algae could be simultaneously stimulated in contrast to the control cultured in nitrogen-replete medium (Figure 11). Although most of the cyanobacteria stimulated the growth and lipid content of the algae later in cultivation, only Nostoc sp. 47-2-1 was able to stimulate both biomass and lipid content of Coelastrum sp. over the entire experimental period. When Nostoc sp. 47-2-1 was inoculated again in co-culture with Coelastrum sp. 46-4 with more replicates, only a significantly higher biomass concentration was observed in the co-culture during the first week while there were no significant differences found between mono- and co-cultures at the end of cultivation (Figure 16). Nile red lipid fluorescence showed no significantly higher fluorescence from the co-culture in contrast to the monoculture (Figure 17). Pairing of Coelastrum sp. 46-4 with Nostoc sp. 47-2-1 demonstrates that algae can be cultivated in co-culture with diazotrohpic organisms with similar biomass and lipid yields without the use of nitrogen. Eliminating nitrogen application by introduction of nitrogensupplementing cyanobacteria into culture can drastically reduce the capital cost of algae production and provide a sustainable practice (Borowitzka and Moheimani 2013). 
Co-culturing of the marine algae Cricosphaera sp. 146-2-9 with three strains of fungi indicated one strain to stimulate the simultaneous increase of growth and lipid productivity, fungus 146-2-F3 which was identified as Fusarium sp. (Figure 12). Co-cultures of Fusarium sp. and Cricosphaera sp. 146-2-9 had an overall increase of $29 \%$ in biomass and $13.5 \%$ in lipid productivity. Although a lowered lipid content was found within the co-cultures, the Nile Red fluorescence intensity of Fusarium sp. and Cricosphaera sp. 146-2-9 demonstrated multiple regions of intensified fluorescence near fungal hyphae (Figure 13). It is suspected that fungi-algae interaction and formations, or pellets, are the result of differential cellular surface charges of either microorganism. In co-culture the positively charged mycelia cells attract negatively charged algae to facilitate interactions (Zhou et al. 2013). With closer contact, possible heightened cellular communications occur that lead to stimulated lipid concentrations in the marine algae. A closer look at the effects of Fusarium exudates on Cricosphaera further elucidated the biomass and lipid stimulations in co-culture.

\subsection{Effect of microorganisms on algae}

In determining the effect of the bacterium Pseudomonas stutzeri and its extracellular compounds on the growth and lipid accumulation within the freshwater Coelastrum sp. 46-4, in addition to co-cultures (M), the algae was inoculated in bacteria cell-free medium (BS) and in nutrient broth (NB). The algae cultured in BS were consistently significantly higher in biomass and lipid productivity (Figure 14). Although the biomass of axenic Coelastrum sp. 46-4 
inoculated in NB was also significantly higher, signifying a mixotrophic alga, little lipid fluorescence was observed from the cells (Figure 15). Various cavities in place of lipid bodies were observed in the algal cells cultured in NB. This proposes that the productivity increase in the BS treatment does not depend on the contents of the nutrient broth medium but the contents of bacterial EPS. These results are the first to indicate that bacterial exudates can account for the simultaneously stimulation of biomass and lipid production in algae (Park et al. 2008). There were no significant differences in biomass and lipid content of the co-culture in contrast to the initial results from the preliminary screening (section 3.3.1). In order to verify these present results, gravimetric analysis was applied to accurately measure biomass and lipid content of the algae in the varying treatments.

In elucidating the effects of Fusarium sp. on marine Cricosphaera sp. 1462-9, the algae was inoculated in fungal cell-free filtrate, marine GPY medium, and co-cultured (M) with live cells as well. Results indicate consistent significantly heightened lipid content within the algae cells cultured in FS as apposed to the monocultures with up to a $120.7 \%$ difference (Figure 18). The algae cells cultured in GPY, although showed improved biomass concentrations, did not develop lipid contents higher than the monoculture or FS treatment. Results indicate that Cricosphaera sp. 146-2-9 may be mixotrophic, but the GPY cannot account for the heightened lipid concentrations found within the algae cultured in fungal exudates. Overwhelmingly high lipid fluorescence was observed from these cells cultured in FS further indicating improved lipid yields (Figure 19). 
Though the co-culture of Fusarium sp. and Cricosphaera sp. 146-2-9 did not indicate significant improved biomass yields but moderate, the lipid content yield is striking enough to consider the effects of fungal exudates in stimulating algal cells in production platforms.

4.4 Gravimetric analysis of biomass and lipid content

A closer look at the dried weight and lipid yield of Coelastrum sp. 46-4 in various treatments (BS, $M$, and NB), revealed a $42.25 \%$ biomass increase of the co-culture in contrast to the control (Figure 20). These gravimetric results coincide with the preliminary screening results (section 3.3.1) but not necessarily section 3.4. These results suggest that different cultivation parameters are going to have substantial effects on co-cultures of microorganism. Since higher biomass is observed in the co-cultures in larger culture schemes, it can be proposed that without the effects of shaking and introduction of air bubbling, there was an amplified relationship between the bacterium and the algae. Since Pseudomonas stutzeri is motile, shaking could have impeded the suspension of the bacterium while bubbling did not (Lalucat et al. 2006). It is already indicated that presence of mutualistic Pseudomonas sp. live cells has drastic effects on algae growth rates (Guo and Tong 2014) and bacterial contact is necessary in order to promote growth (Do Nascimento et al. 2013). Besides biomass, the highest lipid content was observed in the BS treatment with about $35 \%$ of dry biomass, and about $30.7 \%$ of those lipids were FAMEs suitable for use in 
biodiesel. This is the first indication that bacterial exudates account for lipid increases in algal cells. The exudates of other Pseudomonas sp. have indicated positive growth correlation in the green algae Chlorella sp. and that bacterial glycoproteins could play a significant role (Riquelme et al. 1988) or bacteria may provide algae with necessary vitamins for growth (Kazamia et al. 2012). It is also proposed that the reduction of $\mathrm{O}_{2}$ by bacteria through consumption, relieving algae of oxygen damage, could in part cause a rise in algal growth as well (Mouget et al. 1995). In either case, the presence of Pseudomonas stutzeri cells in co-culture with Coelastrum sp. 46-4 stimulated the biomass of the algae and the bacterial exudate accounted for the observed intensified lipid content.

Gravimetric biomass and lipid analysis of co-cultures of Coelastrum sp. 46-4 with diazotrophic Nostoc sp. $47-2-1$ shows a $70 \%$ decrease in the biomass of the co-culture but a $46.7 \%$ increase in lipid content (Figure 21). These results were drastically different from the co-culture experiments in section 3.5. The large difference in biomass concentration of the co-culture in relation to the monoculture is most likely caused by differences in cultivation parameters. It has already been shown that agitation speed during cultivation can have significant effects on the relationship between microorganisms in co-culture (Cheirsilp et al. 2011). Agitation speed during culturing may increase the mass transfer of compounds between the microorganisms in co-culture, and without the added agitation, air bubbling was not adequate enough to keep cyanobacterial cells in suspension to facilitate the growth and communication between algae cells. Results suggest that cultivation of algae without the supplemented nitrogen is 
therefore achievable by co-culturing with $\mathrm{N}_{2}$-fixing cyanobacteria contingent on adequate culture agitation.

When the marine algae Cricosphaera sp. 146-2-9 was cultured in varying treatments (FS, M, and GPY) similar results were obtained from preliminary screening and small-scale experiments. Higher biomass and lipid concentrations were observed in the co-culture of Fusarium $\mathrm{sp}$. and this alga than in monoculture (Figure 22). These results suggest that Fusarium sp. in co-culture with Cricosphaera sp. 146-2-9 can be used to simultaneously amplify biomass and lipid content. The highest lipid content occurred in the FS treatment. The FS treatment had a $35 \%$ lipid content of dried biomass, a high majority of which was comprised of FAMEs. Such high lipid content in FS treatment suggests that fungal extracellular compounds can generate high intracellular lipid concentrations within algae suitable for biodiesel (Figure 22). Since the biomass of this alga was not necessarily productive in GPY, the added compounds could have stunted the growth of the algae and masking the ability of the fungal supernatant to stimulate biomass as well. These results for the first time elucidate how fungal presence and extracellular compounds can promote increased biomass and lipid productivity in algal cells.

\subsection{Conclusions and future prospects}

Isolation, screening and evaluation of South Florida fresh- and saltwater algae resulted in identification of a green alga, Coelastrum sp. $46-4$ and a golden brown haptophyte, Cricosphaera sp. 146-2-9 with moderate biomass and lipid 
production capabilities. Screening of these two algal species with co-cultures of yeast, bacteria, diazotrophic cyanobacteria, and fungi showed that these microorganisms could have either Inhibitory or stimulatory effects on algae. Yeasts had an overall inhibitory effect on both biomass and lipid yield in algae. Conversely, cyanobacteria, heterotrophic bacteria, and fungi showed promising results in promoting algae productivity. When co-culturing diazotrophic cyanobacteria with algae in nitrogen deficient medium, productivity were comparable to monocultures in nitrogen-supplemented medium but were dependent on the agitation speed of the culture. Heterotrophic bacteria Pseudomonas stutzeri and fungus Fusarium sp. were both associated with simultaneous enhanced biomass and lipid productivity in co-culture with Coelastrum sp. 46-4 and Cricosphaera sp. 146-2-9, respectively, through both the presence of microbial cells and the production of extracellular compounds. Understanding the relationship between these microorganisms and their effects on promoting algae growth and lipid metabolism can be applied to strains used in the lab or in mass cultivation, since a large number of these alga are usually xenic in culture (Lang et al. 2011).

Positive correlations results observed in cultures with cell-free bacterial or fungal exudates strongly supports the possibility that extracellular compounds play a significant role in stimulating algae growth and lipid metabolism. Increased lipid content in selected algae in response to co-culture is an impetus for future work geared towards identifying the lipid profiles of those algal strains to determine their full biodiesel potential. And since cell-free microbial exudates 
were found to enhance algae productivity, characterization of the compound/s responsible and elucidating the mechanism involved should be priorities for future research. 


\section{LIST OF REFERENCES}

Adams, C., Godfrey, V., Wahlen, B., Seefeldt, L., \& Bugbee, B. (2013). Understanding precision nitrogen stress to optimize the growth and lipid content tradeoff in oleaginous green microalgae. Bioresource Technology, 131, 188-194.

Anderson, D. M., Glibert, P. M., \& Burkholder, J. M. (2002). Harmful algal blooms and eutrophication: Nutrient sources, composition, and consequences. Estuaries, 25(4), 704-726.

Angelis, S., Novak, A. C., Sydney, E. B., Soccol, V. T., Carvalho, J. C., Pandey, A., ... Soccol, C. R. (2012). Co-Culture of Microalgae, Cyanobacteria, and Macromycetes for Exopolysaccharides Production: Process Preliminary Optimization and Partial Characterization. Applied Biochemistry and Biotechnology, 167(5), 1092-106.

Axler, R. P., Redfield, G. W., \& Goldman, C. R. (1981). The Importance of Regenerated Nitrogen to Phytoplankton Productivity to Phytoplankton Productivity in a Subalpine Lake. Ecology, 62(2), 345-354.

Barnard, D., Casanueva, A., Tuffin, M., \& Cowan, D. (2010). Extremophiles in biofuel synthesis. Environmental Technology, 31(8-9), 871-888.

Becker, E. W. (1994). Microalgae: biotechnology and microbiology. Cambridge University Press, Cambridge, UK

Beer, L. L., Boyd, E. S., Peters, J. W., \& Posewitz, M. C. (2009). Engineering algae for biohydrogen and biofuel production. Current Opinion in Biotechnology, 20(3), 264-271.

Bell, W. H. (1983). Bacterial utilization of algal extracellular products. 3. The specificity of algal-bacterial interaction. Limnology and Oceanography, 28(6), 1131-1143. 
Bell, W. H., \& Sakshaug, E. (1980). Bacterial utilization of algal extracellular products. 2. A kinetic study of natural populations. Limnology and Oceanography, 25(6), 1021-1033.

Bellinger, E.G., Sigee, D.C. (2010). Freshwater algae - identification and use as bioindicators. Wiley-Blackwell, Great Britain.

Bernstein, A.M., Roizen, M.F., Martinez, L. 2014. Purified palmitoleic acid for the reduction of high-sensitivity C-reactive protein and serum lipids: A doubleblinded, randomized, placebo controlled study. Journal of Clinical Lipidology, 8:612-617.

Bolton, John J., What is aquatic botany? - and why algae are plants: the importance of non-taxonomic terms for groups of organisms. Aquatic Botany http://dx.doi.org/10.1016/l.aquabot.2016.02.006

Bonnet, S., Webb, E. A., Panzeca, C., Karl, D. M., Capone, D. G., \& Wilhelmy, S. A. S. (2010). Vitamin B12 excretion by cultures of the marine cyanobacteria Crocosphaera and Synechococcus. Limnology and Oceanography, 55(5), 1959-

Borowitzka, M. A. (1997). Microalgae for aquaculture: Opportunities and constraints. Journal of Applied Phycology, 9(5), 393-401.

Borowitzka, M. A., \& Moheimani, N. R. (2013). Sustainable biofuels from algae. Mitigation and Adaptation Strategies for Global Change, 18(1), 13-25.

Brennan, L., \& Owende, P. (2010). Biofuels from microalgae-A review of technologies for production, processing, and extractions of biofuels and coproducts. Renewable and Sustainable Energy Reviews, 14(2), 557-577.

Brooks, B. W., Lazorchak, J. M., Howard, M. D. A., Johnson, M.-V. V., Morton, S. L., Perkins, D. A. K., ... Steevens, J. A. (2016). Are harmful algal blooms becoming the greatest inland water quality threat to public health and aquatic ecosystems? Environmental Toxicology and Chemistry, 35(1), 6-13. 
Cai, S., Hu, C., \& Du, S. (2007). Comparisons of Growth and Biochemical Composition between Mixed Culture of Alga and Yeast and Monocultures. Journal of Bioscience and Bioengineering, 104(5), 391-397.

Cao, H., Zhang, Z., Wu, X., Miao, X. (2013). Direct Biodiesel Production from Wet Microalgae Biomass of Chlorella pyrenoidosa through In Situ Transesterification. BioMed Research International, 2013, e930686.

Carriquiry, M. A., Du, X., \& Timilsina, G. R. (2011). Second generation biofuels: Economics and policies. Energy Policy, 39(7), 4222-4234.

Cheirsilp, B., Suwannarat, W., \& Niyomdecha, R. (2011). Mixed culture of oleaginous yeast Rhodotorula glutinis and microalga Chlorella vulgaris for lipid production from industrial wastes and its use as biodiesel feedstock. New Biotechnology, 28(4), 362-368.

Chen, C.-Y., Yeh, K.-L., Aisyah, R., Lee, D.-J., \& Chang, J.-S. (2011). Cultivation, photobioreactor design and harvesting of microalgae for biodiesel production: A critical review. Bioresource Technology, 102(1), 71-81.

Chen, Y.-C. (2012). The biomass and total lipid content and composition of twelve species of marine diatoms cultured under various environments. Food Chemistry, 131(1), 211-219.

Chen, W., Zhang, C., Song, L., Sommerfeld, M., \& Hu, Q. (2009). A high throughput Nile red method for quantitative measurement of neutral lipids in microalgae. Journal of Microbiological Methods, 77(1), 41-47.

Chisti, Y. (2013). Constraints to commercialization of algal fuels. Journal of Biotechnology, 167(3), 201-214.

Le Chevanton, M., Garnier, M., Bougaran, G., Schreiber, N., Lukomska, E., Bérard, J.-B., ... Cadoret, J.-P. (2013). Screening and selection of growth-promoting bacteria for Dunaliella cultures. Algal Research, 2(3), 212-222. 
Cho, D.-H., Ramanan, R., Heo, J., Lee, J., Kim, B.-H., Oh, H.-M., \& Kim, H.-S. (2015). Enhancing microalgal biomass productivity by engineering a microalgal-bacterial community. Bioresource Technology, 175, 578-585.

Cole, J. J. (1982). Interactions Between Bacteria and Algae in Aquatic Ecosystems. Annual Review of Ecology and Systematics, 13(1), 291-314.

Congressional Research Service (CRS) (2013). Renewable Fuel Standards (RFS)Overview and Issues. 7-5700, R40155. www.crs.gov

Converti, A., Casazza, A. A., Ortiz, E. Y., Perego, P., \& Del Borghi, M. (2009). Effect of temperature and nitrogen concentration on the growth and lipid content of Nannochloropsis oculata and Chlorella vulgaris for biodiesel production. Chemical Engineering and Processing: Process Intensification, 48(6), 11461151.

Croft, M. T., Lawrence, A. D., Raux-Deery, E., Warren, M. J., \& Smith, A. G. (2005a). Algae acquire vitamin B12 through a symbiotic relationship with bacteria. Nature, 438(7064), 90-93.

Croft, M. T., Warren, M. J., \& Smith, A. G. (2006). Algae Need Their Vitamins. Eukaryotic Cell, 5(8), 1175-1183.

D’Oca, M. G. M., Viêgas, C. V., Lemões, J. S., Miyasaki, E. K., Morón-Villarreyes, J. A., Primel, E. G., \& Abreu, P. C. (2011a). Production of FAMEs from several microalgal lipidic extracts and direct transesterification of the Chlorella pyrenoidosa. Biomass and Bioenergy, 35(4), 1533-1538.

Davies, S. W., Wham, D., Kanke, M. R., \& Matz, M. V. (2016). Ecological factors rather than physical barriers shape genetic structure of algal symbionts in Micronesian corals. bioRxiv, 037994.

Dawson, C. J., \& Hilton, J. (2011). Fertiliser availability in a resource-limited world: Production and recycling of nitrogen and phosphorus. Food Policy, 36, Supplement 1, S14-S22. 
de-Bashan, L. E., Bashan, Y., Moreno, M., Lebsky, V. K., \& Bustillos, J. J. (2002). Increased pigment and lipid content, lipid variety, and cell and population size of the microalgae Chlorella spp. when co-immobilized in alginate beads with the microalgae-growth-promoting bacterium Azospirillum brasilense. Canadian Journal of Microbiology, 48(6), 514-521.

Do Nascimento, M., Dublan, M. de los A., Ortiz-Marquez, J. C. F., \& Curatti, L. (2013). High lipid productivity of an Ankistrodesmus-Rhizobium artificial consortium. Bioresource Technology, 146, 400-407.

Domozych, D. S., Ciancia, M., Fangel, J. U., Mikkelsen, M. D., Ulvskov, P., \& Willats, W. G. T. (2012). The Cell Walls of Green Algae: A Journey through Evolution and Diversity. Frontiers in Plant Science, 3.

Dunahay, T. G., Jarvis, E. E., Dais, S. S., \& Roessler, P. G. (1996). Manipulation of Microalgal Lipid Production Using Genetic Engineering. In C. E. Wyman \& B. H. Davison (Eds.), Seventeenth Symposium on Biotechnology for Fuels and Chemicals (pp. 223-231).

Fukami, K., Nishijima, T., \& Ishida, Y. (1997). Stimulative and inhibitory effects of bacteria on the growth of microalgae. Hydrobiologia, 358(1-3), 185-191.

Gao, Z., Zhao, H., Li, Z., Tan, X., \& Lu, X. (2012). Photosynthetic production of ethanol from carbon dioxide in genetically engineered cyanobacteria. Energy \& Environmental Science, 5(12), 9857-9865.

Griffiths, J. M., Harrison, T. L. S. (2009). Lipid Productivity as a key characteristic for choosing algal species for biodiesel production. J Appl Phycol 21:493-507.

Gultom, S., \& Hu, B. (2013). Review of Microalgae Harvesting via Co-Pelletization with Filamentous Fungus. Energies, 6(11), 5921-5939.

Guo, Z., \& Tong, Y. W. (2014). The interactions between Chlorella vulgaris and algal symbiotic bacteria under photoautotrophic and photoheterotrophic conditions. Journal of Applied Phycology, 26(3), 1483-1492. 
Haas, M. J., \& Wagner, K. (2011). Simplifying biodiesel production: The direct or in situ transesterification of algal biomass. European Journal of Lipid Science and Technology, 113(10), 1219-1229.

Hannon, M., Gimpel, J., Tran, M., Rasala, B., \& Mayfield, S. (2010). Biofuels from algae: challenges and potential. Biofuels, 1(5), 763-784.

Hempel, N., Petrick, I., \& Behrendt, F. (2012). Biomass productivity and productivity of fatty acids and amino acids of microalgae strains as key characteristics of suitability for biodiesel production. Journal of Applied Phycology, 24(6), 14071418.

Higgins, B. T., \& VanderGheynst, J. S. (2014). Effects of Escherichia coli on Mixotrophic Growth of Chlorella minutissima and Production of Biofuel Precursors. PLOS ONE, 9(5), e96807.

Hobbie, J. E., Rublee, P. 1977. Radioisotopestudies of heterotrophic bacteria in aquatic ecosystems. In Aquatic Microbial Communities. ed. J. Cairns, pp.

Hu, Q., Sommerfeld, M., Jarvis, E., Ghirardi, M., Posewitz, M., Seibert, M., \& Darzins, A. (2008). Microalgal triacylglycerols as feedstocks for biofuel production: perspectives and advances. Plant Journal, 54(4), 621-639.

IPCC (2014) Climate Change 2014: Synthesis Report. Contribution of Working Groups I, II and III to the Fifth Assessment Report of the Intergovernmental Panel on Climate Change [Core Writing Team, R.K. Pachauri and L.A. Meyer (eds.)]. IPCC, Geneva, Switzerland, 151 pp.

Islam, M. A., Brown, R. J., Brooks, P. R., Jahirul, M. I., Bockhorn, H., \& Heimann, K. (2015). Investigation of the effects of the fatty acid profile on fuel properties using a multi-criteria decision analysis. Energy Conversion and Management, 98, 340347.

Johnson, M. B., \& Wen, Z. (2009). Production of Biodiesel Fuel from the Microalga Schizochytrium limacinum by Direct Transesterification of Algal Biomass. Energy \& Fuels, 23(10), 5179-5183. 
Jones, J. G. (1972). Studies on Freshwater Bacteria: Association with Algae and Alkaline Phosphatase Activity. Journal of Ecology, 60(1), 59-75.

Karatay, S. E., \& Dönmez, G. (2011). Microbial oil production from thermophile cyanobacteria for biodiesel production. Applied Energy, 88(11), 3632-3635.

Kazamia, E., Czesnick, H., Nguyen, T. T. V., Croft, M. T., Sherwood, E., Sasso, S., ... Smith, A. G. (2012). Mutualistic interactions between vitamin B12-dependent algae and heterotrophic bacteria exhibit regulation. Environmental Microbiology, 14(6), 1466-1476.

Kim, B.-H., Ramanan, R., Cho, D.-H., Oh, H.-M., \& Kim, H.-S. (2014). Role of Rhizobium, a plant growth promoting bacterium, in enhancing algal biomass through mutualistic interaction. Biomass and Bioenergy, 69, 95-105.

Kim, H.-J., Choi, Y.-K., Jeon, H. J., Bhatia, S. K., Kim, Y.-H., Kim, Y.-G., .. Yang, Y.H. (2015). Growth promotion of Chlorella vulgaris by modification of nitrogen source composition with symbiotic bacteria, Microbacterium sp. HJ1. Biomass and Bioenergy, 74, 213-219.

Kitcha, S., \& Cheirsilp, B. (2014a). Enhanced Lipid Production by Co-cultivation and Co-encapsulation of Oleaginous Yeast Trichosporonoides spathulata with Microalgae in Alginate Gel Beads. Applied Biochemistry and Biotechnology, 173(2), 522-534.

Koh, L. P., Miettinen, J., Liew, S. C., \& Ghazoul, J. (2011). Remotely sensed evidence of tropical peatland conversion to oil palm. Proceedings of the National Academy of Sciences, USA, 108(12), 5127-5132.

Kossuga, M. H., Romminger, S., Xavier, C., Milanetto, M. C., Valle, M. Z. do, Pimenta, E. F., Berlinck, R. G. S. (2012). Evaluating methods for the isolation of marine-derived fungal strains and production of bioactive secondary metabolites. Revista Brasileira de Farmacognosia, 22(2), 257-267.

Lalucat, J., Bennasar, A., Bosch, R., García-Valdés, E., \& Palleroni, N. J. (2006). Biology of Pseudomonas stutzeri. Microbiology and Molecular Biology Reviews, 70(2), 510-547. 
Lambert, William Franklin. (2013). Culturing and co-culturing of the nitrogen-fixing cyanobacterium Nodularia in nitrogen-deplete media for biotechnological applications /. UC San Diego: b8126916.

Lang, I., Hodac, L., Friedl, T., \& Feussner, I. (2011). Fatty acid profiles and their distribution patterns in microalgae: a comprehensive analysis of more than 2000 strains from the SAG culture collection. BMC Plant Biology, 11, 124.

Lee, Y.-K. (2001). Microalgal mass culture systems and methods: Their limitation and potential. Journal of Applied Phycology, 13(4), 307-315.

Li, Y., Horsman, M., Wang, B., Wu, N., \& Lan, C. Q. (2008). Effects of nitrogen sources on cell growth and lipid accumulation of green alga Neochloris oleoabundans. Applied Microbiology and Biotechnology, 81(4), 629-36.

Maity, J. P., Bundschuh, J., Chen, C.-Y., \& Bhattacharya, P. (2014). Microalgae for third generation biofuel production, mitigation of greenhouse gas emissions and wastewater treatment: Present and future perspectives - A mini review. Energy, 78, 104-113.

Markou, G., \& Nerantzis, E. (2013). Microalgae for high-value compounds and biofuels production: A review with focus on cultivation under stress conditions. Biotechnology Advances, 31(8), 1532-1542.

Martin, M. A. (2010). First generation biofuels compete. New Biotechnology, 27(5), 596-608.

Mata, T. M., Martins, A. A., \& Caetano, N. S. (2010). Microalgae for biodiesel production and other applications: A review. Renewable and Sustainable Energy Reviews, 14(1), 217-232.

Mimouni, V., Ulmann, L., Pasquet, V., Mathieu, M., Picot, L., Bougaran, G., ... Schoefs, B. (2012). The potential of microalgae for the production of bioactive molecules of pharmaceutical interest. Current Pharmaceutical Biotechnology, 13(15), 2733-2750. 
Mouget, J.-L., Dakhama, A., Lavoie, M. C., \& de la Noüe, J. (1995). Algal growth enhancement by bacteria: Is consumption of photosynthetic oxygen involved? FEMS Microbiology Ecology, 18(1), 35-43.

Narendar, Priyanka, "Screening and Identification of Everglades Algal Isolates for Biodiesel production" (2010). FIU Electronic Theses and Dissertations. Paper 287.

Nguyen, H.T., Park, H., Koster, K.L., Cahoon, R.E., Nguyen, H.T., Shanklin, J., Clemente, T.E., Cahoon, E.B. 2015. Redirection of metabolic flux for high levels of omega-7 monounsaturated fatty acid accumulation in camelina seeds. Plant Biotechnol J., 13:38-50.

Nodine, E. R., \& Gaiser, E. E. (2013). Distribution of Diatoms Along Environmental Gradients in the Charlotte Harbor, Florida (USA), Estuary and Its Watershed: Implications for Bioassessment of Salinity and Nutrient Concentrations. Estuaries and Coasts, 37(4), 864-879.

Nozzi, N. E., Oliver, J. W. K., \& Atsumi, S. (2013). Cyanobacteria as a Platform for Biofuel Production. Frontiers in Bioengineering and Biotechnology, 1.

Okuda, K. (2002). Structure and phylogeny of cell coverings. Journal of Plant Research, 115(4), 283-288.

Papone, T., Kookkhunthod, S., Leesing, R. (2012) Microbial oil production by monoculture and mixed cultures of microalgae and oleaginous yeasts using sugarcane juice as substrate. World Academy of Science, Engineering and Technology 64

Park, Y., Je, K., Lee, K., Jung, S., \& Choi, T. (2008). Growth promotion of Chlorella ellipsoidea by co-inoculation with Brevundimonas sp. isolated from the microalga. Hydrobiologia, 598(1), 219-228.

Peccia, J., Haznedaroglu, B., Gutierrez, J., \& Zimmerman, J. B. (2013). Nitrogen supply is an important driver of sustainable microalgae biofuel production. Trends in Biotechnology, 31(3), 134-138. 
Rikkinen, J. (2015). Cyanolichens. Biodiversity and Conservation, 24(4), 973-993.

Riquelme, C. E., K. Fukami, Y. Ishida (1988). Effects of bacteria on the growth of a marine diatom, Asterionella glacialis. Bulletin of Japanese Society of Microbial Ecology 3: 29-34.

Rodolfi, L., Chini Zittelli, G., Bassi, N., Padovani, G., Biondi, N., Bonini, G., \& Tredici, M. R. (2009). Microalgae for oil: Strain selection, induction of lipid synthesis and outdoor mass cultivation in a low-cost photobioreactor. Biotechnology and Bioengineering, 102(1), 100-112.

Salama, E., Kim, H., Abou-shanab, R. A., I, Ji, M., Oh, Y., ... Jeon, B. (2013). Biomass, lipid content, and fatty acid composition of freshwater Chlamydomonas mexicana and Scenedesmus obliquus grown under salt stress. Bioprocess and Biosystems Engineering, 36(6), 827-33.

Sapp, M., Schwaderer, A. S., Wiltshire, K. H., Hoppe, H., Gerdts, G., \& Wichels, A. (2007). Species-Specific Bacterial Communities in the Phycosphere of Microalgae? Microbial Ecology, 53(4), 683-99.

Sarma, S. J., Brar, S. K., Sydney, E. B., Le Bihan, Y., Buelna, G., \& Soccol, C. R. (2012). Microbial hydrogen production by bioconversion of crude glycerol: A review. International Journal of Hydrogen Energy, 37(8), 6473-6490.

Schenk, P. M. (2012). Isolation and Evaluation of Oil-Producing Microalgae from Subtropical Coastal and Brackish Waters. PLoS ONE, 7(7).

Sharma, K. K., Schuhmann, H., \& Schenk, P. M. (2012). High Lipid Induction in Microalgae for Biodiesel Production. Energies, 5(5), 1532-1553.

Sheehan J., Dunahay T., Benemann J., Roessler P. 1998. A look back at the US Department of Energy's Aquatic Species Program-biodiesel from algae. Report no. NREL/TP-580-24190 National Renewable Energy Laboratory, Golden, Colorado. 
Silva-Benavides, A. M., \& Torzillo, G. (2011). Nitrogen and phosphorus removal through laboratory batch cultures of microalga Chlorella vulgaris and cyanobacterium Planktothrix isothrix grown as monoalgal and as co-cultures. Journal of Applied Phycology, 24(2), 267-276.

Su, C.-H., Chien, L.-J., Gomes, J., Lin, Y.-S., Yu, Y.-K., Liou, J.-S., \& Syu, R.-J. (2010). Factors affecting lipid accumulation by Nannochloropsis oculata in a twostage cultivation process. Journal of Applied Phycology, 23(5), 903-908.

Su, J., Yang, X., Zheng, T., \& Hong, H. (2007). An efficient method to obtain axenic cultures of Alexandrium tamarense-a PSP-producing dinoflagellate. Journal of Microbiological Methods, 69(3), 425-430.

Tai, V., Paulsen, I. T., Phillippy, K., Johnson, D. A., \& Palenik, B. (2009). Wholegenome microarray analyses of Synechococcus- Vibrio interactions. Environmental Microbiology, 11(10), 2698-2709.

Takagi, M., Karseno, \& Yoshida, T. (2006). Effect of salt concentration on intracellular accumulation of lipids and triacylglyceride in marine microalgae Dunaliella cells. Journal of Bioscience and Bioengineering, 101(3), 223-226.

Trexler, J. C., Gaiser, E.E., Kominoski, J.S., Sanchez, J. L. (2015). "The role of periphyton mats in consumer community structure and function in calcareous wetlands: lessons from the Everglades."Microbiology of the Everglades Ecosystem. Science Publications, CRC Press, Boca Raton: 155-179.

Trochine, C., Guerrieri, M. E., Liboriussen, L., Lauridsen, T. L., \& Jeppesen, E. (2014). Effects of nutrient loading, temperature regime and grazing pressure on nutrient limitation of periphyton in experimental ponds. Freshwater Biology, 59(5), 905-917.

U.S. Environmental Protection Agency (2011), Biofuels and the Environment: First Triennial Report to Congress. Office of Research and Development, National Center for Environmental Assessment, Washington, DC; EPA/600/R-10/183F 
U.S. Environmental Protection Agency (U.S. EPA) (2010) Renewable fuel standard program (RFS2) Regulatory Impact Analysis. U.S. EPA, Washington, DC, EPA420-R-10-006

USDA, World Agricultural Supply and Demand Estimates and National Agricultural Statistics Service. Updated March 2015.

Vigeolas, H., Waldeck, P., Zank, T., \& Geigenberger, P. (2007). Increasing seed oil content in oil-seed rape (Brassica napus L.) by over-expression of a yeast glycerol-3-phosphate dehydrogenase under the control of a seed-specific promoter. Plant Biotechnology Journal, 5(3), 431-441.

Wang, R., Tian, Y., Xue, S., Zhang, D., Zhang, Q., Wu, X., ... Cong, W. (2016). Enhanced microalgal biomass and lipid production via co-culture of Scenedesmus obliquus and Candida tropicalis in an autotrophic system. Journal of Chemical Technology \& Biotechnology, 91(5), 1387-1396.

Warabi, Y.; Kusdiana, D. \& Saka, S. (2004). Reactivity of triglycerides and fatty acidsof rapeseed oil in supercritical alcohols. Bioresource Technology,Vol. 9, pp. 283-287.

Watanabe, K., Takihana, N., Aoyagi, H., Hanada, S., Watanabe, Y., Ohmura, N., ... Tanaka, H. (2005). Symbiotic association in Chlorella culture. FEMS Microbiology Ecology, 51(2), 187-196.

Wehr, J. D., \& Sheath, R. G. (Eds.). (2003). Freshwater algae of North America: ecology and classification. Amsterdam ; Boston: Academic Press.

Wiebe, W. J., \& Smith, D. F. (1977). Direct measurement of dissolved organic carbon release by phytoplankton and incorporation by microheterotrophs. Marine Biology, 42(3), 213-223.

Wrede, D., Taha, M., Miranda, A. F., Kadali, K., Stevenson, T., Ball, A. S., \& Mouradov, A. (2014). Co-Cultivation of Fungal and Microalgal Cells as an Efficient System for Harvesting Microalgal Cells, Lipid Production and Wastewater Treatment. PLOS ONE, 9(11). 
Xie, S., Sun, S., Dai, S. Y., \& S.Yuan, J. (2013). Efficient coagulation of microalgae in cultures with filamentous fungi. Algal Research, 2(1), 28-33.

Xin, L., Hong-ying, H., \& Yu-ping, Z. (2011). Growth and lipid accumulation properties of a freshwater microalga Scenedesmus sp. under different cultivation temperature. Bioresource Technology, 102(3), 3098-3102.

Xue, Feiyan, Miao, Jinxin, Zhang, Xu, Tan, Tianwei, (2010). A new strategy for lipid production by Mix cultivation of Spirulina platensis and Rhodotorula glutinis. Applied Biomchem Biotechnol, 160: 498-503.

Yen, H.-W., Chen, P.-W., \& Chen, L.-J. (2015). The synergistic effects for the cocultivation of oleaginous yeast-Rhodotorula glutinis and microalgaeScenedesmus obliquus on the biomass and total lipids accumulation. Bioresource Technology, 184, 148-152.

Zhang, J., \& Hu, B. (2012). A novel method to harvest microalgae via co-culture of filamentous fungi to form cell pellets. Bioresource Technology, 114, 529-535.

Zhou, W., Cheng, Y., Li, Y., Wan, Y., Liu, Y., Lin, X., \& Ruan, R. (2012). Novel Fungal Pelletization-Assisted Technology for Algae Harvesting and Wastewater Treatment. Applied Biochemistry and Biotechnology, 167(2), 214-28. 